\title{
Verossimilhança hierárquica em modelos de fragilidade
}

\author{
William Nilson de Amorim \\ DisSERTAÇÃO APRESENTADA \\ $\mathrm{AO}$ \\ Instituto DE MATEMÁtica E EstatísticA \\ DA \\ Universidade De SÃo PaUlo \\ PARA \\ OBTENÇÃO DO TÍTULO \\ $\mathrm{DE}$ \\ Mestre em CiÊnCIAS \\ Programa: Mestrado em Estatística \\ Orientadora: Profa. Dra. Gisela Tunes
}

Durante o desenvolvimento deste trabalho o autor recebeu auxílio financeiro do CNPq.

São Paulo, março de 2015. 


\section{Verossimilhança hierárquica em modelos de fragilidade}

Esta versão da dissertação contém as correções e alterações sugeridas pela Comissão Julgadora durante a defesa da versão original do trabalho, realizada em 12/02/2015. Uma cópia da versão original está disponível no Instituto de Matemática e Estatística da Universidade de São Paulo.

Comissão Julgadora:

- Prof ${ }^{a}$. Dr ${ }^{a}$. Gisela Tunes (orientadora) - IME-USP

- Prof. Dr. Antonio Carlos Pedroso de Lima - IME-USP

- Prof. Dr. Rinaldo Artes - Insper 
We all make choices.

But in the end, our choices make us.

Andrew Ryan 


\section{Agradecimentos}

São muitas pessoas e momentos importantes... mas fica aqui uma pequena parte disso tudo.

Primeiramente, agradeço muito ao meu pai, Raimundo Notato de Amorim por uma frase que certa vez ele me disse e simplesmente resume o que eu gostaria de dizer aqui: "A maior herança que eu posso te deixar é a educação.".

Ainda primeiramente, agradeço a minha mãe, Ivete da Conceição Cruz, por ter sido mais forte do que eu jamais serei em momentos bem difíceis de nossas vidas. Momentos esses em que eu ainda era muito cego para apreciar tudo o que você fazia, o que me impediu de te dar o devido valor. Mas o tempo passa, a gente cresce e todo e qualquer ressentimento deu lugar ao enorme respeito e gratidão que sinto por ti.

Agradeço aos meus orientadores Antonio Carlos Pedroso de Lima e Gisela Tunes por todas as conversas, discussões, conselhos e lições que transcenderam o âmbito acadêmico e me fizeram um estatístico e uma pessoa melhor. Vocês são exemplos de professores, pesquisadores e mestres.

Agradeço a minha irmã, Amanda Cruz de Amorim, pelo amor e cumplicidade que cresce a cada dia. Você não tem ideia do orgulho que sinto de ti em cada conquista que você alcança.

Agradeço a minha namorada Agatha Sacramento Rodrigues por todo amor, carinho, incentivo, conversas, companheirismo e, principalmente, por todas as risadas neste último ano. Você deixou este caminho percorrido muito mais fácil, muito mais alegre.

Agradeço a Larissa Caroline Vieira Meneghin, antes de mais nada, pela confiança ao ter me alugado um quarto em seu apartamento quando nem mesmo me conhecia. E de estranhos, passamos a amigos e, hoje, eu também agradeço pelas piores piadas que já escutei, pelos magníficos bolos de cenoura e pela ótima amizade.

Agradeço muito aos meus amigos: Felipe Pires Martins ${ }^{1}$ por tantos momentos inesquecivelmente engraçados, pelas discussões sobre futebol e Lost e pela honra de ser seu padrinho de casamento; Hamilton Kazuyuki Tateoka por todas as visitas a sua casa e ao ACENBO, pelos testes de resistência e pelas 67 voltas de circular; Guilherme Biasi pelo companheirismo nas competições de Atletismo, por me ensinar o valor de uma platina e por todas as brincadeiras que fazemos na sua presença ou ausência; Gerson

\footnotetext{
${ }^{1} \mathrm{O}$ Zumbi.
} 
Hitoshi Kumagaia por ser um irmão para mim, com quem sempre pude contar, nas melhores e piores horas; Matheus Kaneji Nagaoka² por ser um irmão para mim, com quem sempre pude contar, nas melhores e piores horas; Eduardo Carvalho da Silva por tantas pizzas compartilhadas, pelas sempre engraçadas partidas de PES e por ser meu colega de quarto por mais tempos que nós dois gostaríamos; Iuri Naoto Nobre Ota pelas conversas, pelos livros e quadrinhos emprestados e pelas tardes de video game, compartilhando comigo uma parte das suas raras horas acordado; e Lennon Luis Rafael Molfetti de Oliveira ${ }^{3}$ simplesmente por existir. Muito obrigado por terem sido uma família para mim nesses 8 anos de IME.

E por fim, agradeço ao acaso por, em uma tarde nada especial de 2006, sem nenhum motivo concreto ou relevante, me fazer escolher Estatística no vestibular. Desde então, pensando em tudo o que vivi e aprendi, em todas as pessoas que conheci e hoje fazem parte da minha vida, posso dizer que essa escolha (quase aleatória) me fez quem sou hoje.

\footnotetext{
${ }^{2} \mathrm{E}$ também à sua família, uma das mais bonitas que já conheci, pela hospitalidade em tantas visitas e pelos convites para tantos almoços e jantares.

${ }^{3}$ Agradeço também a sua família pelo carinho e hospitalidade em tanto churrascos e naquela peculiar noite de Ano Novo.
} 


\section{Resumo}

AMORIM, W. A. Verossimilhança hierárquica em modelos de fragilidade. 2014. Dissertação - Instituto de Matemática e Estatística, Universidade de São Paulo, São Paulo, 2014.

Os métodos de estimação para modelos de fragilidade vêm sendo bastante discutidos na literatura estatística devido a sua grande utilização em estudos de Análise de Sobrevivência. Vários métodos de estimação de parâmetros dos modelos foram desenvolvidos: procedimentos de estimação basados no algoritmo EM, cadeias de Markov de Monte Carlo, processos de estimação usando verossimilhança parcial, verossimilhança penalizada, quasiverossimilhança, entro outros. Uma alternativa que vem sendo utilizada atualmente é a utilização da verossimilhança hierárquica. O objetivo principal deste trabalho foi estudar as vantagens e desvantagens da verossimilhança hierárquica para a inferência em modelos de fragilidade em relação a verossimilhança penalizada, método atualmente mais utilizado. Nós aplicamos as duas metodologias a um banco de dados real, utilizando os pacotes estatísticos disponíveis no software R, e fizemos um estudo de simulação, visando comparar o viés e o erro quadrático médio das estimativas de cada abordagem. Pelos resultados encontrados, as duas metodologias apresentaram estimativas muito próximas, principalmente para os termos fixos. Do ponto de vista prático, a maior diferença encontrada foi o tempo de execução do algorítimo de estimação, muito maior na abordagem hierárquica.

Palavras-chave: Análise de Sobrevivência, modelos de fragilidade, verossimilhança hierárquica, verossimilhança penalizada. 


\section{Abstract}

AMORIM, W. A. Hierarchical likelihood in frailty models. 2014. Dissertação Instituto de Matemática e Estatística, Universidade de São Paulo, São Paulo, 2014.

Estimation procedures for frailty models have been widely discussed in the statistical literature due its widespread use in survival studies. Several estimation methods were developed: procedures based on the EM algorithm, Monte Carlo Markov chains, estimation processes based on parcial likelihood, penalized likelihood and quasi-likelihood etc. An alternative currently used is the hierarchical likelihood. The main objective of this work was to study the hierarchical likelihood advantages and disadvantages for inference in frailty models when compared with the penalized likelihood method, which is the most used one. We applied both approaches to a real data set, using $\mathrm{R}$ packages available. Besides, we performed a simulation study in order to compare the methods through out the bias and the mean square error of the estimators. Both methodologies presented very similar estimates, mainly for the fixed effects. In practice, the great difference was the computational cost, much higher in the hierarchical approach.

Keywords: Survival analysis, frailty models, hierarchical likelihood, penalized likelihood. 


\section{Sumário}

1 Introdução 1

2 O modelo de fragilidade 3

2.1 Estimação por verossimilhança penalizada . . . . . . . . . . . . . 4

2.1.1 O modelo de fragilidade Gama . . . . . . . . . . . . . . . . . 7

2.1.2 Modelo de fragilidade Log-normal . . . . . . . . . . . . . . . . 8

2.2 Estimação por verossimilhança hierárquica . . . . . . . . . . . . . . . . 10

2.2.1 Modificações na abordagem hierárquica . . . . . . . . . . . . . . . . 12

2.3 Testes de hipóteses . . . . . . . . . . . . . . . . . . . . . . . . . . 13

2.4 Modelos com dois ou mais componentes de fragilidade . . . . . . . . . . . . 14

3 Aplicação $\quad 17$

4 Simulação $\quad 25$

4.1 Modelos com um componente de fragilidade . . . . . . . . . . . . 25

4.1.1 Resultados . . . . . . . . . . . . . . . . 26

4.2 Modelos com dois componentes de fragilidade . . . . . . . . . . . . 37

4.2.1 Resultados . . . . . . . . . . . . . . . . . . 38

5 Conclusões $\quad 43$

$\begin{array}{ll}\text { A Expressões do Capítulo 2 } & 45\end{array}$

B Expressões do Capítulo $4 \quad 49$

C Gráficos $\quad 53$

C.1 Gráficos da simulação Gama . . . . . . . . . . . . . . . . . . 53

C.2 Gráficos da simulação Log-normal . . . . . . . . . . . . . . . . . . . . . 62

$\begin{array}{ll}\text { Referências Bibliográficas } & 67\end{array}$ 


\section{Capítulo 1}

\section{Introdução}

Modelos de fragilidade têm sido bastante utilizados em estudos de Análise de Sobrevivência. Eles são uma extensão do modelo de risco proporcionais de Cox (1972) que permitem incorporar possíveis correlações entre os tempos de falha causadas, por exemplo, por um agrupamento natural ou artificial das unidades amostrais ou por tempos medidos em um mesmo indivíduo. A mensuração dessa correlação muitas vezes não é importante para o estudo, mas a falsa suposição de independência pode levar a resultados numericamente errados e conclusões equivocadas.

Como exemplo dentro deste contexto, consideremos um estudo realizado pelo Instituto de Ciências Biomédicas da Universidade de São Paulo (ICB-USP), cujo objetivo era avaliar a memória espacial de ratos com pequenas lesões cerebrais induzidas pelos pesquisadores (Tunes e de Lima, 2005). O experimento consistia em colocar os animais em um tanque com água e medir o tempo até que eles alcançassem uma plataforma fixada em um ponto da piscina. Aqueles que não completassem a tarefa em até 120 segundos tinham seus tempos censurados e eram retirados da água. Esse procedimento foi repetido durante 14 dias, sendo realizadas duas tentativas por dia.

Neste caso, a suposição de independência entre os tempos observados para uma mesma cobaia pode não ser adequada e a correlação entre essas observações deve ser contemplada pelo modelo utilizado na análise. Note que dois componentes de fragilidade podem ser atribuídos nesse problema: um aos tempos medidos no mesmo animal no mesmo dia; e outro aos tempos medidos no mesmo animal em dias diferentes.

A inferência para modelos de fragilidade vem sendo estudada por vários autores. Para o modelo de fragilidade gama, Klein (1992) e Nielsen et al. (1992) desenvolveram procedimentos de estimação baseados no algoritmo EM e Parner (1998) mostrou a consistência e normalidade assintótica da estimação de Nielsen et al. (1992). Ainda para o modelo 
gama, Clayton (1991) e Aslanidou et al. (1998) desenvolveram procedimentos de cadeias de Markov de Monte Carlo, que podem ser computacionalmente intensos. Para o modelo de fragilidade lognormal, McGilchrist e Aisbett (1991) e McGilchrist (1993) desenvolveram um processo de estimação usando a verosimilhança parcial de Cox (1972), mas esse método não lidava com empates entre os tempos de sobrevivência.

Posteriormente, foi proposta a estimação de modelos de fragilidade por meio de verossimilhança penalizada para diferentes distribuições do efeito aleatório, como a Gama (Therneau e Grambsch, 2000) e a Log-normal (Ripatti e Palmgren, 2000). Hoje em dia, a verossimilhança penalizada é amplamente utilizada para estimação nos modelos de fragilidade em Análise de Sobrevivência.

No contexto de modelos lineares generalizados, existe também literatura extensa sobre incorporação de efeitos aleatórios. Métodos de estimação propostos incluem quadratura gaussiana (Pinheiro e Chao, 2006), métodos baseados na quasi-verossimilhança penalizada (PQL), proposta por Breslow e Clayton (1993), entre outros. Lee e Nelder (1996) propuseram o uso da verossimilhança hierárquica (ou h-likelihood) para a inferência de modelos lineares generalizados com efeitos aleatórios.

A utilização da verossimilhança hierárquica em modelos de fragilidade em análise de sobrevivência foi considerada por Ha et al. (2001), que mostraram que essa aproximação fornece uma abordagem unificada com várias distribuições de fragilidade, incluindo a Gama e a Log-normal. Mais recentemente, Ha et al. (2010) investigaram a relação entre as verossimilhanças penalizada, marginal e hierárquica e verificaram que o estimador de máxima verossimilhança pode ser viciado no caso de modelos de fragilidade semiparamétricos, além de propor metodologia para reduzir o viés.

Este trabalho teve como objetivo principal o estudo da verossimilhança hierárquica no contexto de modelos de fragilidade e sua comparação com a verossimilhança penalizada, a técnica mais difundida. Além de um estudo teórico, realizamos estudos de simulação e uma análise dos pacotes computacionais disponíveis no software estatístico $\mathrm{R}$, visando estabelecer as vantagens e desvantagens de cada metodologia. Como motivação prática, utilizamos o banco de dados do estudo do ICB-USP, sendo que a ele aplicamos e comparamos as técnicas aqui estudadas.

No próximo capítulo, abordaremos os aspectos teóricos da caracterização e estimação dos modelos de fragilidade. No capítulo 3, se encontram os resultados da análise do exemplo prático e, no capítulo 4, os resultados do estudo de simulação. Por fim, no capítulo 5, apresentaremos as principais conclusões deste trabalho. 


\section{Capítulo 2}

\section{O modelo de fragilidade}

Nas décadas de 70 e 80, motivados por estudos epidemiológicos e experimentos clínicos, surgiram muitos trabalhos discutindo metodologias para lidar com dados de sobrevida correlacionados (Clayton, 1978; Oakes, 1982; Johansen, 1983; Hougaard, 1986; Oakes, 1989). O principal desafio era incorporar, aos modelos usuais, a correlação causada por tempos de falha medidos em indivíduos de uma mesma família, clínica, região ou mesmo tempos medidos em um mesmo indivíduo. Uma das principais abordagens na época, além da utilização de famílias de distribuições bivariadas, foi a extensão do modelo de riscos proporcionais de Cox, que mais tarde deu origem aos modelos de fragilidade.

O termo fragilidade foi introduzido por Vaupel et al. (1979) e, desde então, passou a indicar o risco comum entre indivíduos de um mesmo grupo de apresentar o evento de interesse. Hoje em dia, os modelos de fragilidade se tornaram peça fundamental na análise de dados de sobrevida multivariados e, nas últimas duas décadas, os métodos de estimação para esses modelos vêm sendo bastante discutidos.

O modelo de fragilidade deve ser construído com base na estrutura de correlação dos dados. Devido a sua simplicidade, abordaremos aqui o modelo de fragilidade compartilhada (shared frailty model), em que cada indivíduo pertence a um único grupo, cada grupo compartilha uma mesma fragilidade e os termos de fragilidade entre grupos diferentes são considerados independentes. Para informações sobre outros modelos de fragilidade, consultar Colosimo e Giolo (2006).

Formalmente, considere $\mathbf{T}_{i}=\left(T_{i 1}, \ldots, T_{i n_{i}}\right)^{T}$ os tempos de sobrevivência dos $n_{i}$ indivíduos pertencentes ao $i$-ésimo grupo e $z_{i}$ a fragilidade não-observada associada a eles. Assumiremos que, condicionalmente a $z_{i}$, os componentes de $\mathbf{T}_{i}$ são independentes e caracterizados pela função de taxa de falha dada por 


$$
\lambda_{i j}\left(t \mid \mathbf{x}_{\mathbf{i j}}, z_{i}\right)=\lambda_{0}(t) \exp \left\{\mathbf{x}_{\mathbf{i j}}^{\mathbf{T}} \boldsymbol{\beta}\right\} z_{i}
$$

para $i=1, \ldots, q$ e $j=1, \ldots, n_{i}$. Aqui, $\lambda_{i j}\left(t \mid \mathbf{x}_{\mathbf{i j}}, z_{i}\right)$ é a função de risco do $j$-ésimo indivíduo do $i$-ésimo grupo condicionada à fragilidade $z_{i}$ e a um vetor $\mathbf{x}_{\mathbf{i j}}=\left(x_{i j 1}, \ldots, x_{i j p}\right)^{T}$ de covariáveis, $\lambda_{0}(t)$ é uma função de risco basal desconhecida e $\boldsymbol{\beta}$ um vetor de parâmetros $p \times 1$. Neste trabalho, também assumiremos que a variável de fragilidade $z_{i}$, com densidade $f_{z_{i}}\left(z_{i}, \xi\right)$, não varia com o tempo. Por simplicidade, muitas vezes o modelo

$$
\lambda_{i j}\left(t \mid \mathbf{x}_{\mathbf{i j}}, w_{i}\right)=\lambda_{0}(t) \exp \left\{\mathbf{x}_{\mathbf{i j}}^{\mathbf{T}} \boldsymbol{\beta}+w_{i}\right\}
$$

é considerado no lugar de (2.0.1). Observe que os dois modelos são equivalentes, sendo $w_{i}=\log z_{i}$, com densidade $f_{w_{i}}\left(w_{i}, \xi\right)$.

Para dados censurados, sendo $C_{i j}$ a censura do $j$-ésimo indivíduo do $i$-ésimo grupo, este modelo assume ainda que, condicionalmente a $z_{i}$, o mecanismo de censura é independente e não-informativo (Kalbfleisch e Prentice, 2002; Lawless, 2002).

Dentre as distribuições de fragilidade discutidas na literatura, destacam-se a Gama e a Log-normal. Na prática, a escolha dessa distribuição é feita com base na tratabilidade matemática e na disponibilidade computacional, sendo que, na grande maioria das vezes, a qualidade do ajuste não é investigada. Algumas técnicas gráficas e numéricas de diagnóstico foram propostas para o modelo de fragilidade Gama (Glidden, 1999; Cui e Sun, 2004; Geerdens et al., 2013), mas ainda é uma área que demanda maiores estudos.

A seguir, abordaremos dois métodos de estimação para modelos de fragilidade: a verossimilhança penalizada e a verossimilhança hierárquica. Em sequência, apresentaremos testes de hipóteses para a significância dos efeitos fixos e para a existência de correlação entre os tempos. Por fim, discutiremos brevemente a extensão do modelo (2.0.2) para mais de um componente de fragilidade.

\subsection{Estimação por verossimilhança penalizada}

A verossimilhança penalizada surgiu como um método para estimação de densidades (Good e Gaskins, 1971) e passou a ser uma importante ferramenta para estimação de modelos mistos. A utilização dessa verossimilhança em modelos de fragilidade foi proposta por Therneau e Grambsch (2000), para o modelo Gama, e por Ripatti e Palmgren (2000) para o modelo Log-normal. Na última década, a abordagem penalizada se tornou a metodologia mais utilizada na estimação de modelos de fragilidade, substituindo os algoritmos EM de Nielsen et al. (1992) e Klein (1992).

Considerando o modelo de fragilidade dado por (2.0.2), a abordagem penalizada con- 
siste em utilizar a verossimilhança parcial de Cox considerando $\mathbf{w}$ como um vetor de parâmetros, cuja estimação é controlada por uma função de penalidade $g(\mathbf{w}, \xi)$. Em geral, essa função associa pesos pequenos para valores distantes do zero, evitando um superajuste do modelo.

A função de log-verossimilhança parcial penalizada é dada por

$$
\ell_{P P}=\ell_{P P}(\boldsymbol{\beta}, \mathbf{w})=\ell_{P}(\boldsymbol{\beta}, \mathbf{w})-g(\mathbf{w}, \xi)
$$

em que

$$
\ell_{P}(\boldsymbol{\beta}, \mathbf{w})=\sum_{i=1}^{q} \sum_{j=1}^{n_{i}} \delta_{i j}\left[\mathbf{x}_{i j}^{T} \boldsymbol{\beta}+w_{i}-\log \left(\sum_{R\left(t_{i j}\right)} \exp \left\{\mathbf{x}_{i j}^{T} \boldsymbol{\beta}+w_{i}\right\}\right)\right]
$$

é a log-verossimilhança parcial de Cox e $g(\mathbf{w}, \xi)$ é uma função de penalidade controlada pelo parâmetro $\xi$. Em geral, como veremos nas próximas seções, essa função é tomada como o núcleo da densidade da variável de fragilidade. Na expressão $(2.1 .2), \delta_{i j}$ é o indicador de falha do $j$-ésimo indivíduo pertencente ao $i$-ésimo grupo e $R\left(t_{i j}\right)$ é o conjunto de índices das observações em risco no momento exatamente anterior ao tempo de falha $t_{i j}$.

Os estimadores de $\boldsymbol{\beta}$ e w são encontrados pela maximização de (2.1.1). Como a função de penalidade não envolve as covariáveis, as funções escore para $\boldsymbol{\beta}$ são as mesmas do modelo de Cox usual, tratando w como um offset. Assim, para $k=1, \ldots, p$,

$$
U\left(\beta_{k}\right)=\frac{\partial \ell_{P P}}{\partial \beta_{k}}=\sum_{i=1}^{q} \sum_{j=1}^{n_{i}} \delta_{i j}\left[x_{i j k}-\frac{\sum_{R\left(t_{i j}\right)} x_{i j k} \exp \left\{\mathbf{x}_{i j}^{T} \boldsymbol{\beta}+w_{i}\right\}}{\sum_{R\left(t_{i j}\right)} \exp \left\{\mathbf{x}_{i j}^{T} \boldsymbol{\beta}+w_{i}\right\}}\right] .
$$

Já as funções escore de $w_{k}$, para $k=1, \ldots, q$, são dadas por

$$
U\left(w_{k}\right)=\frac{\partial \ell_{P P}}{\partial w_{k}}=d_{k}-\sum_{i=1}^{q} \sum_{j=1}^{n_{i}} \delta_{i j}\left[\frac{\sum_{R\left(t_{k j}\right)} \exp \left\{\mathbf{x}_{k j}^{T} \boldsymbol{\beta}+w_{k}\right\}}{\sum_{R\left(t_{i j}\right)} \exp \left\{\mathbf{x}_{i j}^{T} \boldsymbol{\beta}+w_{i}\right\}}\right]-\frac{\partial g(\mathbf{w}, \xi)}{\partial w_{k}}
$$

sendo $d_{k}=\sum_{j=1}^{n_{k}} \delta_{k j}$ o número de falhas do grupo $k$.

O processo de estimação de $\xi$ considera a maximização da log-verossimilhança marginal, dada por 


$$
\ell_{m}\left(\lambda_{0}, \boldsymbol{\beta}, \xi\right)=\log \int_{-\infty}^{\infty} L\left(\lambda_{0}, \boldsymbol{\beta}, \mathbf{w}, \xi\right) d \mathbf{w}
$$

avaliada em $\hat{\lambda}_{0}$ e $\hat{\boldsymbol{\beta}}$. Em geral, utiliza-se como estimativa para $\hat{\lambda}_{0}$ uma extensão do estimador não-paramétrico de Breslow (1974). Observe que $L\left(\lambda_{0}, \boldsymbol{\beta}, \mathbf{w}, \xi\right)$ representa a verossimilhança completa, que pode ser decomposta como

$$
\begin{aligned}
L\left(\lambda_{0}, \boldsymbol{\beta}, \mathbf{w}, \xi\right) & =L\left(\lambda_{0}, \boldsymbol{\beta}, \xi \mid \mathbf{w}\right) \prod_{i=1}^{q} f\left(w_{j}, \xi\right)= \\
& =\prod_{i=1}^{q} \prod_{j=1}^{n_{i}}\left(\lambda_{i j}(t)\right)^{\delta_{i j}} \exp \left\{-\int_{0}^{t} \lambda_{i j}(u) d u\right\} \prod_{i=1}^{q} f\left(w_{j}, \xi\right) .
\end{aligned}
$$

Em termos mais simples, a estimação de $\xi$ envolve a maximização da log-verossimilhança marginal perfilada $\ell_{m}(\xi)=\ell_{m}\left(\hat{\lambda}_{0}, \hat{\boldsymbol{\beta}}, \xi\right)$, que resulta na função escore

$$
U(\xi)=\frac{\partial \ell_{m}(\xi)}{\partial \xi}
$$

Quando $\ell_{m}(\xi)$ não pode ser obtida de forma analítica, o método de Laplace para aproximação de integrais (Barndorff-Nielsen e Cox, 1989) pode ser aplicado em (2.1.3), resultando na verossimilhança marginal perfilada ajustada de Cox e Reid (1987).

Um algoritmo iterativo deve ser utilizado para resolver as equações escore de $\boldsymbol{\beta}, \mathbf{w}$ e $\xi$. Como passo inicial, $\boldsymbol{\beta}$ é estimado considerando $\mathbf{w}$ como um offset e $\xi$ fixo ${ }^{1}$. Em seguida, as fragilidades são avaliadas dados os valores de $\hat{\boldsymbol{\beta}}$ calculados inicialmente. Esse procedimento é realizado até alcançar a convergência, o que finaliza o loop interno. No segundo passo, $\xi$ é estimado dado os valores de $\hat{\boldsymbol{\beta}}$ e $\hat{\mathbf{w}}$. O algoritmo, então, volta ao primeiro passo e reavalia $\boldsymbol{\beta}$ e w utilizando agora o valor calculado de $\hat{\xi}$. Esses dois passos são repetidos até a convergência, dando fim ao loop externo.

A verossimilhança penalizada pode ser maximizada pelo algoritmo de Newton-Raphson. Além dos vetores escore $U(\boldsymbol{\beta}), U(\mathbf{w})$ e $U(\xi)$, esse procedimento também requer a matriz hessiana da log-verossimilhança parcial penalizada, expressa por

$$
\mathbf{H}(\boldsymbol{\beta}, \mathbf{w})=\left(\begin{array}{cc}
-\frac{\partial^{2} \ell_{P P}}{\partial \boldsymbol{\beta}^{2}} & -\frac{\partial^{2} \ell_{P P}}{\partial \boldsymbol{\beta} \partial \mathbf{w}} \\
-\frac{\partial^{2} \ell_{P P}}{\partial \mathbf{w} \partial \boldsymbol{\beta}} & -\frac{\partial^{2} \ell_{P P}}{\partial \mathbf{w}^{2}}
\end{array}\right)=\left(\begin{array}{ll}
\mathbf{H}_{11} & \mathbf{H}_{12} \\
\mathbf{H}_{21} & \mathbf{H}_{22}
\end{array}\right)=\mathcal{I}+\left(\begin{array}{cc}
0 & 0 \\
0 & g^{\prime \prime}
\end{array}\right) .
$$

${ }^{1}$ Valores iniciais devem ser especificados para $\mathbf{w}$ e $\xi$. 
Aqui, $\mathcal{I}=\mathcal{I}(\boldsymbol{\beta}, \mathbf{w})$ é a matriz de informação do modelo usual de Cox.

\subsubsection{O modelo de fragilidade Gama}

A popularidade do modelo de fragilidade Gama para a análise de dados de sobrevida correlacionados teve início com os algoritmos EM de Klein (1992) e Nielsen et al. (1992). A escolha dessa distribuição, além de conduzir a uma expressão analítica para a função de verossimilhança marginal (2.1.3), traz grande facilidade na implementação desses algoritmos.

Therneau e Grambsch (2000) e Therneau et al. (2003) propuseram a utilização da verossimilhança penalizada para a estimação desses modelos e desenvolveram um algoritmo mais eficiente do que aqueles criados por Klein (1992) e Nielsen et al. (1992). Os autores também demonstraram que as soluções obtidas pela abordagem penalizada coincidiam com as do algoritmo EM, resultado que manteve a popularidade do modelo Gama ao longo dos anos.

Assumindo que $z$ tenha distribuição $\operatorname{Gama}(1 / \xi, 1 / \xi)$, sendo $E(z)=1$ e $\operatorname{Var}(z)=\xi$, temos que

$$
f_{z}(z, \xi)=\frac{\frac{1}{\xi} \bar{\xi}}{\Gamma\left(\frac{1}{\xi}\right)} z^{\frac{1}{\xi}-1} \exp \left\{-\frac{z}{\xi}\right\}
$$

e

$$
f_{w}(w, \xi)=\frac{\frac{1}{\xi} \bar{\xi}}{\Gamma\left(\frac{1}{\xi}\right)} \exp \left\{\frac{w}{\xi}-\frac{\exp \{w\}}{\xi}\right\} .
$$

Therneau et al. (2003) propuseram utilizar uma função de penalidade derivada do logaritmo da densidade de $w$, dada por

$$
g(\mathbf{w}, \xi)=-\frac{1}{\xi} \sum_{j=1}^{q}\left(w_{j}-\exp \left\{w_{j}\right\}\right) .
$$

Eles mostraram que, ao utilizar essa função de penalidade, a solução das equações escore para estimação de $\boldsymbol{\beta}$ e w coincidia com a solução do algoritmo EM para um valor fixado de $\xi$. Isto equivale a dizer que as equações escores da verossimilhança parcial penalizada e da verosimilhança marginal, apesar de serem diferentes, têm mesma solução.

Como discutido anteriormente, a estimação do parâmetro $\xi$ é dada pela maximização da log-verossimilhança marginal perfilada $\ell_{m}(\xi)$. Para o modelo com fragilidade Gama, Therneau e Grambsch (2000) demonstraram que 


$$
\ell_{m}(\xi)=\ell_{p}(\hat{\mathbf{w}}, \hat{\boldsymbol{\beta}})+\sum_{j=1}^{q}\left[\frac{1}{\xi}-\left(d_{j}+\frac{1}{\xi}\right) \log \left(d_{j}+\frac{1}{\xi}\right)-\frac{1}{\xi} \log \xi+\log \left(\frac{\Gamma\left(d_{j}+\frac{1}{\xi}\right)}{\Gamma\left(\frac{1}{\xi}\right)}\right)\right]
$$

Observe que essa expressão para $\ell_{m}(\xi)$ não envolve a função de risco basal, mas depende de $\hat{\mathbf{w}}$. Isso se deve à substituição de $\lambda_{0}(t)$ pelo estimador de Breslow, que, por sua vez, é função de $\hat{\boldsymbol{\beta}}$ e $\hat{\mathbf{w}}$. Therneau et al. (2003) também sugeriram a utilização de $\ell_{m}(\xi)+$ $\sum_{j=1}^{q} d_{j}$ no lugar de $\ell_{m}(\xi)$, pois essa log-verossimilhança perfilada converge para $\ell_{P}(\hat{\boldsymbol{\beta}})-$ $\sum_{j=1}^{q} d_{j}$ quando a variância do efeito aleatório vai para zero. Assim, segundo eles, essa modificação torna a verossimilhança maximizada de um modelo de fragilidade com $\xi$ pequeno comparável com a de um modelo sem termo de fragilidade.

A substituição de $\lambda_{0}(t)$ pelo estimador de Breslow no cálculo de $\ell_{m}(\xi)$ aumenta rapidamente o número de parâmetros de pertubação conforme o tamanho da amostra cresce, causando finite-sample bias (Rondeau et al., 2003). Ha et al. (2010) sugeriram a utilização de uma função de verossimilhança perfilada ajustada para reduzir esse viés na estimação de $\xi$. Essa função, derivada da aproximação de Laplace de segunda ordem para a verossimilhança marginal, será melhor discutida na Seção 2.2.

No software R, a estimação do modelo de fragilidade Gama via verossimilhança penalizada está implementada na função $\operatorname{coxph}()$ do pacote survival.

\subsubsection{Modelo de fragilidade Log-normal}

A utilização da distribuição Log-normal para a fragilidade foi sugerida por McGilchrist e Aisbett (1991) e expandida por Ripatti e Palmgren (2000). O surgimento desse modelo foi motivado principalmente pela necessidade de contemplar estruturas de correlação mais complicadas, que poderiam ser mais facilmente modeladas pela matriz de covariâncias de uma distribuição Normal multivariada do que por extensões do modelo Gama.

O procedimento de Ripatti e Palmgren (2000) assume que w tem distribuição Normal $q$-variada com vetor de médias $\mathbf{0}_{\mathbf{q}}$ e matriz de covariâncias $\mathbf{D}(\xi)$. No modelo de fragilidade compartilhada $(2.0 .2), w_{1}, \ldots, w_{q}$ são variáveis independentes e identicamente distribuídas, o que implica em $\mathbf{D}(\xi)=\xi \mathbf{I}_{\mathbf{q} \times \mathbf{q}}$.

Escrevendo a densidade de $w_{i}$ como

$$
f_{w}\left(w_{i}, \xi\right)=\frac{1}{\sqrt{2 \pi \xi}} \exp \left\{-\frac{w_{i}^{2}}{2 \xi}\right\}
$$

os autores demostraram que, utilizando o método de Laplace para aproximação de inte- 
grais (Barndorff-Nielsen e Cox, 1989), a log-verossimilhança marginal (2.1.3) poderia ser aproximada por

$$
\begin{aligned}
\ell_{m}\left(\lambda_{0}(t), \boldsymbol{\beta}, \xi\right) \approx & \log L\left(\lambda_{0}(t), \boldsymbol{\beta}, \tilde{\mathbf{w}}, \xi\right)-g(\tilde{\mathbf{w}}, \xi)- \\
& -\frac{q}{2} \log \xi-\frac{1}{2} \log (\operatorname{det}[\mathbf{K}(\boldsymbol{\beta}, \tilde{\mathbf{w}}, \xi)])
\end{aligned}
$$

em que $\tilde{\mathbf{w}}$ é solução de

$$
\frac{\partial \log L\left(\lambda_{0}(t), \boldsymbol{\beta}, \tilde{\mathbf{w}}, \xi\right)}{\partial \tilde{\mathbf{w}}}=0
$$

e

$$
\mathbf{K}(\boldsymbol{\beta}, \tilde{\mathbf{w}}, \xi)=\frac{\partial^{2} \log L\left(\lambda_{0}(t), \boldsymbol{\beta}, \tilde{\mathbf{w}}, \xi\right)}{\partial \tilde{\mathbf{w}}^{2}} .
$$

Se considerarmos $\xi$ conhecido e w um outro conjunto de parâmetros associados a efeitos fixos, os dois primeiros termos em (2.1.10) corresponderiam a uma log-verossimilhança penalizada, sendo $L\left(\lambda_{0}(t), \boldsymbol{\beta}, \mathbf{w}, \xi\right)$ a verossimilhança completa do modelo usual de Cox e $g(\mathbf{w}, \xi)$ uma função de penalidade dada por

$$
g(\mathbf{w}, \xi)=\frac{1}{2 \xi} \sum_{i=1}^{q} w_{i}^{2} .
$$

Observe que, assim como no modelo Gama, $g(\mathbf{w}, \xi)=\log f_{w}\left(w_{i}, \xi\right)$. De acordo com Ripatti e Palmgren (2000), maximizar essa log-verossimilhança penalizada seria equivalente a maximizar a log-verossimilhança parcial penalizada. Portanto, ignorando os dois últimos termos $^{2}$ em (2.1.10), os autores sugeriram utilizar a $\ell_{P P}$ dada em (2.1.1) para estimar $\boldsymbol{\beta}$ e w.

Para a estimação de $\xi$, Ripatti e Palmgren (2000) sugeriram a utilização da aproximação da verossimilhança marginal (2.1.10), substituindo-se $L\left(\lambda_{0}(t), \boldsymbol{\beta}, \mathbf{w}, \xi\right)$ por $\ell_{P}(\boldsymbol{\beta}, \mathbf{w})$ e avaliada em $(\hat{\boldsymbol{\beta}}, \hat{\mathbf{w}})$. Desenvolvendo essa expressão, eles mostraram que a equação de estimação resultante é dada por

$$
\hat{\xi}=\frac{\sum_{j=1}^{q} \hat{w}_{j}+t\left(\mathbf{H}_{22}^{-1}\right)}{q},
$$

em que $t\left(\mathbf{H}_{22}^{-1}\right)$ é o traço de $\mathbf{H}_{22}^{-1}=\mathbf{H}_{22}^{-1}(\hat{\mathbf{w}})$, sendo $\mathbf{H}_{22}$ dada por (2.1.6). Observe que,

\footnotetext{
${ }^{2}$ Segundo Ripatti e Palmgren (2000), ignorar os dois últimos termos de (2.1.10) na estimação de $\boldsymbol{\beta}$ e w pode levar a perda de informação. No entanto, os autores mostraram a partir de estudos de simulação que, em geral, esse procedimento gerava boas estimativas para $\boldsymbol{\beta}$.
} 
devido a utilização da log-verossimilhança parcial de Cox, (2.1.10) passa a não envolver $\lambda_{0}(t)$. Portanto, ao contrário do que foi discutido para o modelo Gama, esse procedimento elimina o viés assintótico causado pela estimação do termo não-paramétrico.

No software estatístico R, a abordagem penalizada para o modelo Log-normal está disponível nas funções coxph() e coxme() das bibliotecas survival e coxme, respectivamente.

\subsection{Estimação por verossimilhança hierárquica}

A utilização da verossimilhança hierárquica de Lee e Nelder (1996) para a estimação de modelos de fragilidade foi proposta por Ha et al. (2001) e, como vantagem, trouxe uma abordagem unificada para diferentes distribuições da variável de fragilidade e um algoritmo mais eficiente do que os algoritmos EM. Inicialmente, apresentaremos o procedimento de Lee e Nelder (1996) aplicado à estimação de modelos de fragilidade. Em seguida, serão discutidas modificações da verossimilhança hierárquica, propostas por Ha e Lee (2005), Ha et al. (2010) e Ha et al. (2012).

Considerando novamente o modelo em (2.0.2), a log-verossimilhança hierárquica é definida por

$$
h=h\left(\lambda_{0}, \mathbf{w}, \boldsymbol{\beta}, \xi\right)=\log L\left(\lambda_{0}, \mathbf{w}, \boldsymbol{\beta}, \xi\right)=\log L\left(\lambda_{0}, \boldsymbol{\beta}, \xi \mid \mathbf{w}\right)+\sum_{i=1}^{q} \log f\left(w_{i}, \xi\right),
$$

em que $L\left(\Lambda_{0}, \mathbf{w}, \boldsymbol{\beta}, \xi\right)$ é a verossimilhança completa dada por (2.1.4). Sendo $y_{(1)}<y_{(2)}<$ $\ldots<y_{(l)}$ os $l$ tempos de falha distintos e $d_{(k)}$ o número de falhas em $y_{(k)}$, temos

$$
\begin{aligned}
h= & \log L\left(\lambda_{0}, \boldsymbol{\beta}, \xi \mid \mathbf{w}\right)+\sum_{i=1}^{q} \log \left(f\left(w_{i}, \xi\right)\right)= \\
= & \sum_{i=1}^{q} \sum_{j=1}^{n_{i}}\left[\delta_{i j}\left(\log \lambda_{0}\left(y_{i j}\right)+\mathbf{x}_{\mathbf{i j}}^{\mathbf{T}} \boldsymbol{\beta}+w_{i}\right)-\right. \\
& \left.-\Lambda_{0}\left(y_{i j}\right) \exp \left\{\mathbf{x}_{\mathbf{i j}}^{\mathbf{T}} \boldsymbol{\beta}+w_{i}\right\}\right]+\sum_{i=1}^{q} \log \left(f\left(w_{i}, \xi\right)\right) \\
= & \sum_{k=1}^{l} d_{(k)} \log \lambda_{0}\left(y_{(k)}\right)+\sum_{i=1}^{q} \sum_{j=1}^{n_{i}} \delta_{i j}\left(\mathbf{x}_{\mathbf{i j}}^{\mathbf{T}} \boldsymbol{\beta}+w_{i}\right)- \\
& -\sum_{i=1}^{q} \sum_{j=1}^{n_{i}} \Lambda_{0}\left(y_{i j}\right) \exp \left\{\mathbf{x}_{\mathbf{i j}}^{\mathbf{T}} \boldsymbol{\beta}+w_{i}\right\}+\sum_{i=1}^{q} \log \left(f\left(w_{i}, \xi\right)\right),
\end{aligned}
$$


sendo $y_{i j}=\min \left(t_{i j}, c_{i j}\right)$ e $\Lambda_{0}\left(y_{i j}\right)=\int_{0}^{y_{i j}} \lambda_{0}(u) d u$.

O processo de estimação tem, como passo inicial, o cálculo dos estimadores de máxima verossimilhança hierárquica para $\beta$ e $w$ a partir da verossimilhança hierárquica perfilada

$$
h^{*}=h^{*}(\mathbf{w}, \boldsymbol{\beta})=h\left(\mathbf{w}, \boldsymbol{\beta}, \hat{\Lambda}_{0}\right)
$$

em que

$$
\hat{\Lambda}_{0}(t)=\sum_{k: y_{(k)} \leq t} \hat{\lambda}_{0}\left(y_{(k)}\right)=\sum_{k: y_{(k)} \leq t}\left\{\frac{d_{(k)}}{\sum_{R\left(y_{(k)}\right)} \exp \left(\mathbf{x}_{\mathbf{i}}^{\mathbf{T}} \boldsymbol{\beta}+w_{i}\right)}\right\} .
$$

Observe que $\hat{\Lambda}_{0}(t)$ é uma extensão do estimador de Breslow (1974) para a função de risco acumulada basal e é calculado pela resolução da equação escore $\partial h / \partial \lambda_{0}\left(y_{(k)}\right)=0$.

Assim como na abordagem penalizada, as equações escore

$$
\frac{\partial h^{*}}{\partial \boldsymbol{\beta}}=0 \quad \text { e } \quad \frac{\partial h^{*}}{\partial \mathbf{w}}=0
$$

podem ser resolvidas pelo algoritmo de Newton-Raphson. Neste caso, a matriz de informação observada utilizada pelo algoritmo é dada por

$$
J\left(h^{*}, \boldsymbol{\beta}, \mathbf{w}\right)=-\frac{\partial^{2} h^{*}}{\partial(\boldsymbol{\beta}, \mathbf{w})^{2}} .
$$

A estimação do parâmetro $\xi$ envolve a maximização da verossimilhança hierárquica perfilada ajustada de Lee e Nelder (1996), que neste contexto é definida por

$$
h_{A}(\xi \mid \hat{\boldsymbol{\beta}}, \hat{\mathbf{w}})=h^{*}(\hat{\boldsymbol{\beta}}, \hat{\mathbf{w}})-\frac{1}{2} \log \left(\operatorname{det}\left\{\frac{J\left(h^{*}, \hat{\boldsymbol{\beta}}, \hat{\mathbf{w}}\right)}{2 \pi}\right\}\right) .
$$

O desenvolvimento da expressão (2.2.5) é dado no Apêndice I.

Dessa forma, o estimador para $\xi$ é obtido resolvendo iterativamente a equação

$$
\frac{\partial h_{A}(\hat{\xi})}{\partial \hat{\xi}}=0
$$

sendo que $\hat{\boldsymbol{\beta}}$ e $\hat{\mathbf{w}}$ são reavaliados a cada iteração.

A estimação por verossimilhança hierárquica foi implementada no software $R$ com algumas modificações em relação ao apresentado nesta seção. O algoritmo se encontra disponível no pacote frailtyHL por meio da função frailtyHL() e contempla os modelos Gama e Log-normal. 


\subsubsection{Modificações na abordagem hierárquica}

O procedimento de estimação hierárquico foi implementado com algumas modificações em relação ao método inicialmente proposto por Ha et al. (2001). Basicamente, essas modificações têm como objetivo a redução do viés em cenários com tamanho de amostra pequeno ou altas proporções de censura e se baseiam na utilização da aproximação de Laplace de primeira ou segunda ordem para a verossimilhança marginal (2.1.3).

Para o modelo Gama e Log-normal, Lee et al. (2006) e Ha et al. (2010) discutiram a relação entre a abordagem penalizada e hierárquica, mostrando que $h^{*}$ pode ser escrita como

$$
h^{*}=\ell_{P P}+\sum_{k} d_{(k)}\left(\log d_{(k)}-1\right) .
$$

A demonstração desse resultado se encontra no Apêndice A. Essa expressão implica que $h^{*}$ e $\ell_{P P}$ são proporcionais, pois o segundo termo em (2.2.6) não depende de parâmetros de interesse. Portanto, o procedimento hierárquico pode ser realizado substituindo-se $h^{*}$ por $\ell_{P P}$, uma vez que a verossimilhança parcial penalizada não envolve a estimação de $\lambda_{0}(t)$.

Para a estimação de $\boldsymbol{\beta}$, Ha et al. (2010) sugeriram a maximização da aproximação de Laplace de primeira ordem para a verossimilhança marginal dada por

$$
p_{\mathbf{w}}\left(\ell_{P P}\right)=\ell_{P P}(\boldsymbol{\beta}, \hat{\mathbf{w}})-\frac{1}{2} \log \left(\operatorname{det}\left\{\frac{J\left(\ell_{P P}, \hat{\mathbf{w}}\right)}{2 \pi}\right\}\right)
$$

sendo

$$
J\left(\ell_{P P}, \mathbf{w}\right)=\frac{\partial^{2} \ell_{P P}}{\partial \mathbf{w}^{2}}
$$

e $\hat{\mathbf{w}}$ tal que $\partial \ell_{P P} / \partial \mathbf{w}=0$ (ver Apêndice A). Observe que essa aproximação é análoga àquela proposta por Ripatti e Palmgren (2000) para o modelo Log-normal. Em geral, $\ell_{P P}$ leva a estimativas não-enviesadas para $\beta$. A utilização de $p_{\mathbf{w}}\left(\ell_{P P}\right)$ é interessante (para a redução de viés) em cenários com tamanho de amostra pequeno ou alta proporção de censura.

Para a estimação de $\xi$, Ha e Lee (2005) e Ha et al. (2010) sugeriram aproximações de Laplace de primeira ou segunda ordem para a verossimilhança parcial restrita de $\xi$ (Cox e Reid, 1987), dadas, respectivamente, por

$$
p_{\boldsymbol{\beta}, \mathbf{w}}\left(\ell_{P P}\right)=\left[\ell_{P P}+\frac{1}{2} \log \operatorname{det}\left\{2 \pi J^{-1}\left(\ell_{P P}, \boldsymbol{\beta}, \mathbf{w}\right)\right\}\right]_{\boldsymbol{\beta}=\hat{\boldsymbol{\beta}}, \mathbf{w}=\hat{\mathbf{w}}}
$$


e

$$
s_{\boldsymbol{\beta}, \mathbf{w}}\left(\ell_{P P}\right)=p_{\boldsymbol{\beta}, \mathbf{w}}\left(\ell_{P P}\right)-\frac{F\left(\ell_{P P}\right)}{24},
$$

em que

$$
\begin{gathered}
J\left(\ell_{P P}, \boldsymbol{\beta}, \mathbf{w}\right)=\frac{\partial^{2} \ell_{P P}}{\partial(\boldsymbol{\beta}, \mathbf{w})^{2}} \\
F\left(\ell_{P P}\right)=\operatorname{tr}\left[-\left(3 \frac{\partial^{4} \ell_{P P}}{\partial \mathbf{w}^{4}}+5 \frac{\partial^{3} \ell_{P P}}{\partial \mathbf{w}^{3}} J^{-1}\left(\ell_{P P}, \mathbf{w}\right) \frac{\partial^{3} \ell_{P P}}{\partial \mathbf{w}^{3}}\right) J^{-2}\left(\ell_{P P}, \mathbf{w}\right)\right]_{\mathbf{w}=\hat{\mathbf{w}}}
\end{gathered}
$$

e $(\hat{\boldsymbol{\beta}}, \hat{\mathbf{w}})$ são tais tal que $\partial \ell_{P P} / \partial(\hat{\boldsymbol{\beta}}, \hat{\mathbf{w}})=0$. Note que $p_{\boldsymbol{\beta}, \mathbf{w}}\left(\ell_{P P}\right)$ é a verossimilhança perfilada ajustada (2.2.5) desenvolvida no Apêndice A. Para o modelo Gama, a maximização dessas aproximações no lugar da verossimilhança marginal é uma alternativa para redução do viés gerado pela estimação do termo não-paramétrico $\lambda_{0}$. Ha et al. (2012) sugere a utilização de $p_{\boldsymbol{\beta}, \mathbf{w}}\left(\ell_{P P}\right)$ para o modelo Log-normal e $s_{\boldsymbol{\beta}, \mathbf{w}}\left(\ell_{P P}\right)$ para o modelo Gama.

Na função frailtyHL() estão implementadas as verossimilhanças $\ell_{P P}$ e $p_{\mathbf{w}}\left(\ell_{P P}\right)$ para a estimação de $\boldsymbol{\beta}$ e $p_{\boldsymbol{\beta}, \mathbf{w}}\left(\ell_{P P}\right)$ e $s_{\boldsymbol{\beta}, \mathbf{w}}\left(\ell_{P P}\right)$ para a estimação de $\xi$.

\subsection{Testes de hipóteses}

No contexto de modelos de fragilidade, o teste de Wald é o mais utilizado para avaliar o efeito das covariáveis. Para hipóteses do tipo $H_{0}: \boldsymbol{\beta}=\boldsymbol{\beta}_{\mathbf{0}}$, a estatística de Wald é dada por

$$
W=\left(\hat{\boldsymbol{\beta}}-\boldsymbol{\beta}_{\mathbf{0}}\right)^{T} \mathbf{H}_{\mathbf{1 1}}\left(\hat{\boldsymbol{\beta}}-\boldsymbol{\beta}_{\mathbf{0}}\right)
$$

e tem distribuição assintótica $\chi_{p}^{2}$. No software $\mathrm{R}$, este teste foi implementado nas funções $\operatorname{coxph}(), \operatorname{coxme}()$ e frailtyHL().

Em geral, também é interessante testar se há correlação entre as observações de um mesmo grupo. Essa dúvida pode ser traduzida, em termos estatísticos, pela hipótese $H_{0}: \xi=0$, que pode ser testada pelo teste da razão de verossimilhanças, dado por

$$
R V=2\left[\log L_{1}(\hat{\boldsymbol{\beta}}, \hat{\mathbf{w}}, \hat{\xi})-\log L_{0}(\hat{\boldsymbol{\beta}})\right]
$$

em que $L_{1}(\cdot)$ é a verossimilhança utilizada para estimar $\xi$ e $L_{0}(\cdot)$ é a verossimilhança do modelo usual de Cox. Como a hipótese nula está na fronteira do espaço paramétrico, a 
distribuição assintótica de $R V$ é dada pela mistura 50:50 de uma $\chi_{0}^{2}$ e uma $\chi_{1}^{2}$ (Lee et al., 2006). Portanto, o cálculo do valor p nesse caso é dado por

$$
P\left(R V>R V_{\text {obs }}\right)=\frac{1}{2} P\left(\chi_{0}^{2}>R V_{\text {obs }}\right)+\frac{1}{2} P\left(\chi_{1}^{2}>R V_{o} b s\right)=\frac{1}{2} P\left(\chi_{1}^{2}>R V_{o} b s\right) .
$$

\subsection{Modelos com dois ou mais componentes de fragili- dade}

Quando mais de uma fonte de correlação é detectada, o modelo de fragilidade pode ser facilmente generalizado para incorporar mais de um componente aleatório (Hougaard, 2000). Neste caso, a correlação entre as variáveis de fragilidade também pode ser especificada.

O modelo (2.0.2) pode ser reescrito como

$$
\lambda_{i j}(t \mid \mathbf{X}, \mathbf{A}, \mathbf{w})=\lambda_{0}(t) \exp \{\mathbf{X} \boldsymbol{\beta}+\mathbf{A} \mathbf{w}\}
$$

em que $\mathbf{X}$ é uma matrix modelo $n \times p$ associada aos efeitos fixos e $\mathbf{A}=\left(a_{j k}\right)$ é uma matriz modelo $n \times q$ associada à fragilidade $\mathbf{w}=\left(w_{1}, w_{2}, \ldots, w_{q}\right)^{T}$, sendo

$$
a_{j k}= \begin{cases}1, & \text { se a } j \text {-ésima observação pertence ao grupo } k ; \text { e } \\ 0, & \text { caso contrário. }\end{cases}
$$

Considerando $w^{(1)}, w^{(2)}, \ldots, w^{(r)}$ variáveis aleatórias independentes, podemos generalizar (2.4.1) para incluir $r$ componentes de fragilidade, resultando no seguinte modelo

$$
\lambda_{i j}(t \mid \mathbf{X}, \mathbf{A}, \mathbf{w})=\lambda_{0}(t) \exp \left\{\mathbf{X} \boldsymbol{\beta}+\mathbf{A}_{1} \mathbf{w}^{(1)}+\mathbf{A}_{2} \mathbf{w}^{(2)}+\ldots+\mathbf{A}_{r} \mathbf{w}^{(r)}\right\}
$$

em que $\mathbf{A}_{k}$ é a matriz modelo $n \times q_{k}$ associada ao vetor de fragilidades $\mathbf{w}^{(k)}, k=1, \ldots, r$. Agora, sendo

$$
\mathbf{A}=\left(\mathbf{A}_{1}, \mathbf{A}_{2}, \ldots, \mathbf{A}_{r}\right) \quad \text { e } \quad \mathbf{w}=\left(\begin{array}{c}
\mathbf{w}^{(1)} \\
\mathbf{w}^{(2)} \\
\vdots \\
\mathbf{w}^{(r)}
\end{array}\right)
$$

temos que (2.4.1) pode ser escrito como (2.4.2) e, dessa forma, a metodologia para es- 
timação de modelos com mais de um termo de fragilidade é uma extensão direta dos resultados apresentados anteriormente para modelos com apenas um termo. Observe que, neste caso, o processo de estimação envolve $\boldsymbol{\xi}=\left(\xi_{1}, \xi_{2}, \ldots, \xi_{r}\right)$, em que $\xi_{k}$ é o parâmetro associado ao vetor de fragilidades $\mathbf{w}^{(k)}$.

Se $\mathbf{w}^{(k)} \sim N_{q_{k}}\left(\mathbf{0}, \xi_{k} \mathbf{I}\right)$ então $\mathbf{w}$ em (2.4.2) tem distribuição normal multivariada com vetor de médias $\mathbf{0}$ e matriz de covariâncias $\mathbf{G}$. Note que $\mathbf{G}$ é uma matriz diagonal em blocos, dada por

$$
\left(\begin{array}{cccc}
\xi_{\mathbf{1}} \mathbf{I} & \mathbf{0} & \mathbf{0} & \mathbf{0} \\
\mathbf{0} & \xi_{2} \mathbf{I} & \mathbf{0} & \mathbf{0} \\
\vdots & \vdots & \ddots & \vdots \\
\mathbf{0} & \mathbf{0} & \mathbf{0} & \xi_{r} \mathbf{I}
\end{array}\right)
$$

e pode ser facilmente modificada para contemplar possíveis estruturas de correlação entre os componentes de fragilidade. No entanto, como a inclusão dessa correlação leva a inúmeros outros resultados e cenários a serem estudados, neste trabalho consideraremos apenas o caso independente.

As funções coxme() e frailtyHL() permitem o ajuste de modelos com o mais de um componente de fragilidade. A função coxme(), no entanto, só está implementada para o modelo Log-normal, enquanto a frailtyHL() também permite a distribuição Gama. 
16 O MODELO DE FRAGILIDADE 


\section{Capítulo 3}

\section{Aplicação}

Como motivação prática deste trabalho, vamos retomar o estudo conduzido pelo ICBUSP apresentado na primeira seção. A análise destes dados teve início com o ajuste de modelos mais simples, que foram sendo modificados para contemplar a estrutura de correlação dos dados e atender o objetivo da pesquisa. Apresentaremos nesta seção os principais modelos ajustados, sempre analisando as diferenças entre os resultados da abordagem penalizada e hierárquica.

O banco de dados do estudo é composto por 32 ratos divididos em 3 grupos: um controle, um grupo com lesão na região dorsal e um com lesão na região ventral. Como cada cobaia foi avaliada duas vezes por dia durante 14 dias, podemos considerar duas fontes de correlação: entre os tempos de um mesmo rato e entre os tempos de um mesmo rato medidos em um mesmo dia.

O risco de uma cobaia apresentar o evento no instante $t$ pode ser interpretado como a taxa instantânea de chegada na plataforma. Assim, podemos pensar na função de risco como o desempenho de cada rato, condicional às covariáveis e aos componentes de fragilidade. Portanto, se o rato A apresenta um risco de apresentar o evento $10 \%$ maior que o risco do rato $\mathrm{B}$, diremos que o desempenho do rato A é $10 \%$ melhor que o desempenho do rato B. Observe que, na Seção 2, consideramos componentes de fragilidade induzidos pela correlação de indivíduos pertencentes a um mesmo grupo. Neste experimento, a fragilidade é induzida por tempos medidos em um mesmo indivíduo. Embora a formulação do modelo seja a mesma em ambos os casos, a interpretação dos coeficientes, aqui, será feita considerando o contexto de medidas repetidas.

Descritivamente, podemos observar pela Figura 3.1 que o tempo de falha mediano diminui com o passar dos dias, indicando presença de memória espacial nos três grupos. Na segunda tentativa, o grupo Controle apresentou menores tempos medianos na maioria 
dos dias. Já na primeira tentativa, nenhum grupo apresentou melhores resultados do que os demais. Como apenas as tentativas que ultrapassam 120 segundos são censuradas, podemos observar pela Figura 3.2 que a proporção de censura também diminui ao longo dos dias. Ao todo, aproximadamente 14,40\% dos tempos foram censurados.

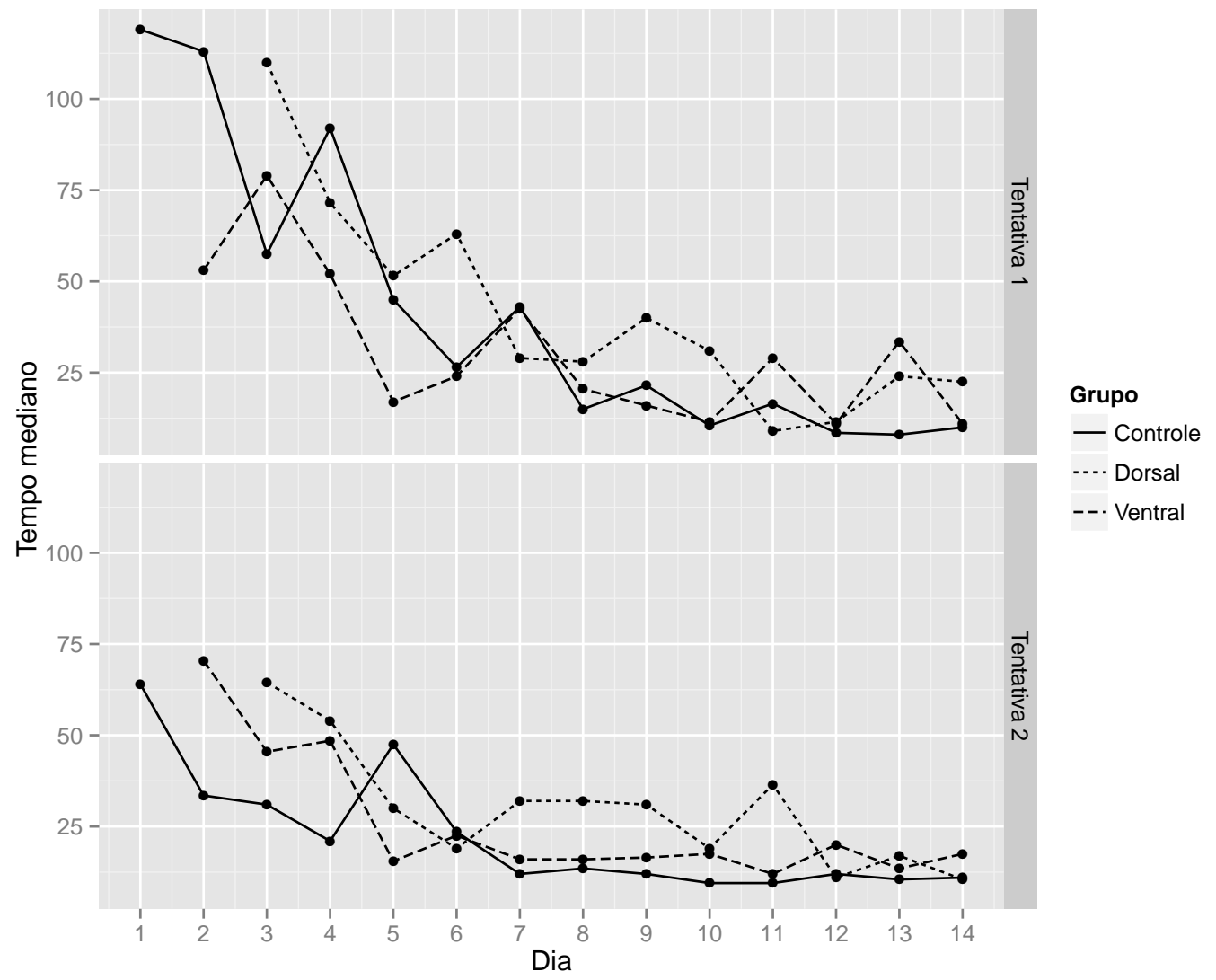

Figura 3.1: Tempo de falha mediano em cada dia calculado pelo estimador de KaplanMeier.

Para o ajuste dos modelos, vamos considerar $\mathbf{x}_{i j k}=\left(x_{1 i}, x_{2 i}, x_{3 i j k}\right)$ o vetor de covariáveis associado a $k$-ésima tentativa do $j$-ésimo dia do $i$-ésimo rato, sendo $x_{1 i}$ e $x_{2 i}$ variáveis separadoras de grupo dadas por

$$
x_{1 i}= \begin{cases}1, & \text { se o } i \text {-ésimo rato pertence ao grupo dorsal } \\ 0, & \text { caso contrário }\end{cases}
$$

$\mathrm{e}$

$$
x_{2 i}= \begin{cases}1, & \text { se o } i \text {-ésimo rato pertence ao grupo ventral } \\ 0, & \text { caso contrário. }\end{cases}
$$

Já $x_{3 i j k}$ é uma variável indicadora que recebe o valor 1 se o tempo medido é referente 


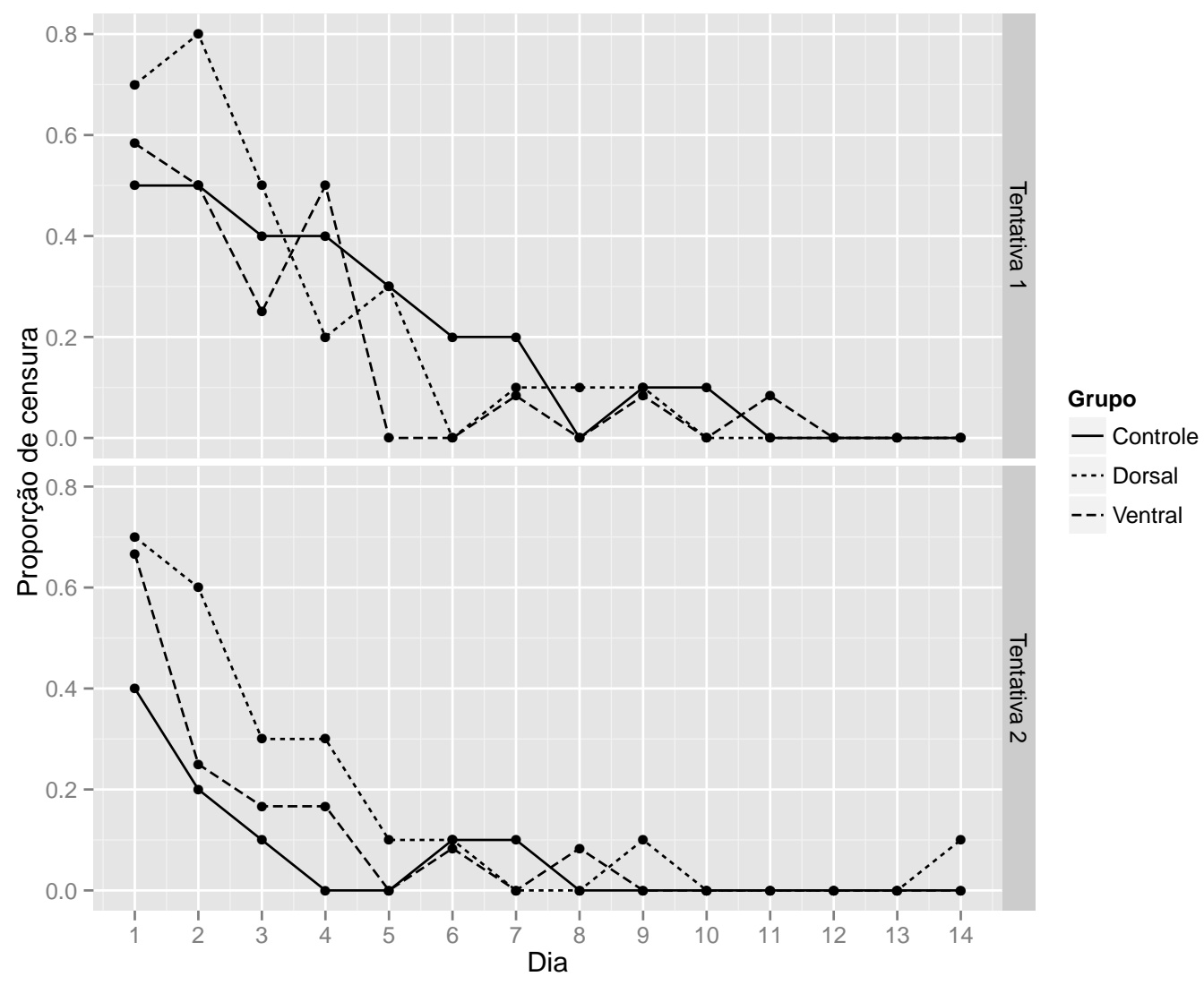

Figura 3.2: Proporção de censura em cada dia.

à segunda tentativa do dia ou 0 caso contrário. Sendo assim, como abordagem inicial, ajustamos dois modelos de fragilidade com a seguinte formulação:

$$
\lambda_{i j k}\left(t \mid \mathbf{x}_{i j k}, w_{i}\right)=\lambda_{0}(t) \exp \left\{\mathbf{x}_{\mathbf{i j k}}^{\mathbf{T}} \boldsymbol{\beta}+w_{i}\right\}
$$

para $i=1, \ldots, 32, j=1, \ldots, 14$ e $k=1,2$, em que $w_{i}$ corresponde ao termo fragilidade associado ao $i$-ésimo animal, sendo $\exp \left\{w_{i}\right\} \sim \operatorname{Gama}(1 / \xi, 1 / \xi)$ para o Modelo I e $w_{i} \sim$ $\mathrm{N}\left(0, \sigma^{2}\right)$ para o Modelo II. Observe que consideramos apenas a correlação entre as medidas de um mesmo rato, ignorando, neste primeiro momento, a correlação de tempos medidos em um mesmo dia.

Podemos observar, pela Tabela 3.1, que o Modelo I (Gama) e o Modelo II (Lognormal), em ambas as abordagens, apresentaram resultados bem próximos, indicando piores desempenhos no grupo dorsal (em relação ao grupo controle) e na primeira tentativa (em relação à segunda). O parâmetro de fragilidade foi considerado significativo em todos os casos, sugerindo que realmente há correlação entre os tempos de um mesmo rato. Quando comparamos a abordagem penalizada e hierárquica para um mesmo modelo, 
Tabela 3.1: Estimativas, com respectivo erro-padrão e significância, dos parâmetros dos Modelos I e II. As funções coxph() e coxme() não disponibilizam o erro-padrão da estimativa do parâmetro associado à fragilidade, sendo que, neste caso, o valor p é calculado a partir do teste de razão de verossimilhanças.

\begin{tabular}{c|cc|cc|cc|}
\hline \multirow{2}{*}{ Parâmetro } & \multicolumn{2}{|c|}{ Estimativa } & \multicolumn{2}{|c|}{ Erro-padrão } & \multicolumn{2}{|c}{ Valor p } \\
\cline { 2 - 7 } & PL & HL & PL & HL & PL & HL \\
\hline \hline \multicolumn{7}{c}{ Modelo I (Gama) } \\
\hline Dorsal & $-0,330$ & $-0,316$ & 0,118 & 0,122 & 0,005 & 0,008 \\
\hline Ventral & $-0,122$ & $-0,118$ & 0,112 & 0,115 & 0,280 & 0,305 \\
\hline Tentativa & 0,241 & 0,238 & 0,073 & 0,073 & 0,001 & 0,001 \\
\hline Fragilidade & 0,026 & 0,031 & - & 0,019 & 0,016 & 0,009 \\
\hline Dorsal & $-0,331$ & $-0,325$ & 0,123 & 0,121 & 0,007 & 0,007 \\
\hline Ventral & $-0,121$ & $-0,118$ & 0,117 & 0,115 & 0,280 & 0,306 \\
\hline Tentativa & 0,241 & 0,237 & 0,073 & 0,073 & 0,001 & 0,001 \\
\hline Fragilidade & 0,033 & 0,031 & - & 0,019 & 0,026 & 0,010 \\
\hline \hline
\end{tabular}

também encontramos resultados numericamente bem semelhantes.

Considerando agora as duas fontes de correlação descritas anteriormente, ajustamos o seguinte modelo de fragilidade (Modelo III):

$$
\lambda_{i j k}\left(t \mid \mathbf{x}_{i j k}, w_{i}, v_{i j}\right)=\lambda_{0}(t) \exp \left\{\mathbf{x}_{\mathbf{i j k}}^{\mathbf{T}} \boldsymbol{\beta}+w_{i}+v_{i j}\right\}
$$

em que $w_{i}$ corresponde ao componente de fragilidade associado às medidas do $i$-ésimo animal e $v_{i j}$ ao componente associado às medidas do $i$-ésimo animal no $j$-ésimo dia. Aqui, utilizamos apenas a distribuição Log-normal para os termos aleatórios pois as funções coxph() e coxme() não permitem o ajuste de modelos Gama com dois ou mais componentes de fragilidade. Pela Tabela 3.2, observamos que este modelo também aponta piores desempenhos no grupo dorsal e na primeira tentativa. Em relação aos componentes de fragilidade, a associação entre medidas de um mesmo rato se mostrou apenas marginal- 
mente significativa, indicando uma possível independência entre tempos medidos em dias diferentes.

Tabela 3.2: Estimativas, com respectivo erro-padrão e significância, dos parâmetros do modelo III. As funções coxph () e coxme() não disponibilizam o erro-padrão da estimativa do parâmetro associado à fragilidade, sendo que, neste caso, o valor p é calculado a partir do teste de razão de verossimilhanças.

\begin{tabular}{c|cc|cc|cc}
\hline \multirow{2}{*}{ Parâmetro } & \multicolumn{2}{|c|}{ Estimativa } & \multicolumn{2}{c|}{ Erro-padrão } & \multicolumn{2}{c}{ Valor p } \\
\cline { 2 - 8 } & PL & HL & PL & HL & PL & HL \\
\hline Dorsal & $-0,510$ & $-0,484$ & 0,174 & 0,168 & 0,003 & 0,004 \\
\hline Ventral & $-0,198$ & $-0,183$ & 0,165 & 0,160 & 0,230 & 0,254 \\
\hline Tentativa & 0,300 & 0,288 & 0,080 & 0,079 & $<0,001$ & $<0,001$ \\
\hline Fragilidade (rato) & 0,044 & 0,046 & - & 0,039 & 0,086 & 0,064 \\
\hline Fragilidade (dia) & 0,842 & 0,692 & - & 0,137 & $<0,001$ & $<0,001$ \\
\hline
\end{tabular}

Nos modelos ajustados até agora, foi possível avaliar se há melhor desempenho entre a primeira e segunda tentativa de um mesmo dia, mas não se há melhora ao longo dos 14 dias. Sendo assim, acrescentamos ao modelo final (Modelo IV) um efeito fixo representando o dia. Além disso, seguindo os modelos ajustados até aqui, os grupos controle e ventral também não apresentaram diferença de desempenho neste modelo, o que nos conduziu à decisão de juntar esses níveis, passando a ter apenas os grupos controle-ventral e dorsal. Dessa maneira, a formulação deste último modelo é dada por

$$
\lambda_{i j k}\left(t \mid \mathbf{x}_{i j k}, w_{i}, v_{i j}\right)=\lambda_{0}(t) \exp \left\{\mathbf{x}_{\mathbf{i j k}}^{\mathbf{T}} \boldsymbol{\beta}+w_{i}+v_{i j}\right\},
$$

sendo $\mathbf{x}_{\mathbf{i j k}}=\left(x_{1 i}, x_{3 k}, \mathbf{x}_{\mathbf{4} \mathbf{j}}^{\mathbf{T}}\right), \operatorname{com} \mathbf{x}_{4 \mathbf{j}}^{\mathbf{T}}=\left(x_{4 j}^{(2)}, x_{4 j}^{(3)}, \ldots, x_{4 j}^{(14)}\right)$ e $x_{4 j}^{(l)}$ igual a 1 se $l=j$ e 0 caso contrário. Assim como no Modelo III, consideramos $w_{i}$ e $v_{i j}$ com distribuição Log-normal.

Pelos resultados da abordagem penalizada apresentados na Tabela 3.3, para tempos medidos na mesma tentativa de um mesmo dia, o desempenho de cobaias com lesão Dorsal é 33,0\% menor do que o desempenho de cobaias no grupo Controle ou com lesão Ventral. Considerando agora ratos de um mesmo grupo, o desempenho na segunda tentativa do dia é 29,3\% maior do que o desempenho da primeira. Esses valores ficaram bem próximos aos estimados pela abordagem hierárquica: $32,2 \%$ e 28,7\%, respectivamente. Também podemos observar que, em geral, o desempenho melhora com o passar dos dias, sendo 
Tabela 3.3: Estimativas, com respectivo erro-padrão e significância, dos parâmetros do Modelo IV. As funções coxph () e coxme() não disponibilizam o erro-padrão da estimativa do parâmetro associado à fragilidade, sendo que, neste caso, o valor p é calculado a partir do teste de razão de verossimilhanças.

\begin{tabular}{|c|c|c|c|c|c|c|}
\hline \multirow{2}{*}{ Parâmetro } & \multicolumn{2}{|c|}{ Estimativa } & \multicolumn{2}{|c|}{ Erro-padrão } & \multicolumn{2}{|c|}{ Valor $\mathrm{p}$} \\
\hline & PL & HL & PL & HL & PL & HL \\
\hline Dorsal & $-0,401$ & $-0,389$ & 0,157 & 0,155 & 0,011 & 0,012 \\
\hline Tentativa & 0,257 & 0,252 & 0,077 & 0,076 & $<0,001$ & $<0,001$ \\
\hline Dia 2 & 0,513 & 0,508 & 0,284 & 0,282 & 0,071 & 0,072 \\
\hline Dia 3 & 0,996 & 0,991 & 0,270 & 0,269 & $<0,001$ & $<0,001$ \\
\hline Dia 4 & 1,086 & 1,082 & 0,269 & 0,268 & $<0,001$ & $<0,001$ \\
\hline Dia 5 & 1,628 & 1,616 & 0,265 & 0,263 & $<0,001$ & $<0,001$ \\
\hline Dia 6 & 1,783 & 1,770 & 0,263 & 0,261 & $<0,001$ & $<0,001$ \\
\hline Dia 7 & 1,869 & 1,851 & 0,265 & 0,262 & $<0,001$ & $<0,001$ \\
\hline Dia 8 & 2,329 & 2,295 & 0,266 & 0,263 & $<0,001$ & $<0,001$ \\
\hline Dia 9 & 2,236 & 2,206 & 0,265 & 0,263 & $<0,001$ & $<0,001$ \\
\hline Dia 10 & 2,466 & 2,431 & 0,265 & 0,263 & $<0,001$ & $<0,001$ \\
\hline Dia 11 & 2,513 & 2,480 & 0,265 & 0,263 & $<0,001$ & $<0,001$ \\
\hline Dia 12 & 2,756 & 2,711 & 0,266 & 0,264 & $<0,001$ & $<0,001$ \\
\hline Dia 13 & 2,449 & 2,419 & 0,264 & 0,262 & $<0,001$ & $<0,001$ \\
\hline Dia 14 & 2,772 & 2,724 & 0,266 & 0,264 & $<0,001$ & $<0,001$ \\
\hline Fragilidade (rato) & 0,111 & 0,107 & - & 0,043 & $<0,001$ & $<0,001$ \\
\hline Fragilidade (dia) & 0,179 & 0,157 & - & 0,064 & 0,002 & 0,004 \\
\hline
\end{tabular}


que o desempenho em uma das tentativas do último dia é cerca de $1500 \%$ melhor que o desempenho referente a essa mesma tentativa do primeiro dia. Os dois componentes de fragilidade foram considerados significativos, indicando que os tempos são correlacionadas não só por serem medidos no mesmo rato, mas também por serem medidos no mesmo dia.

Um próximo passo nessa análise seria avaliar se há associação entre os componentes de fragilidade. Na prática, isso seria equivalente a avaliar se cobaias que apresentam correlação alta (ou baixa) entre as duas tentativas de um mesmo dia tendem a manter essa correlação nas tentativas de dias diferentes. Como esse tipo de estrutura não foi abordada neste estudo, deixamos essa análise como sugestão para trabalhos futuros.

Vale ressaltar que, apesar de não termos explicitado os resultados, não encontramos interação entre as variáveis grupo e tentativa, grupo e dia e dia e tentativa. Além disso, tendo em vista o objetivo deste trabalho e a ausência de técnicas bem estabelecidas, não fizemos análise do ajuste do modelo.

A análise destes dados foi realizada no software estatístico R. Baseando-se no capítulo anterior, na Tabela 3.4 estão listadas as funções e verossimilhanças utilizadas para estimar cada um dos modelos apresentados.

Tabela 3.4: Funções do software R e verossimilhanças utilizadas para ajustar os modelos I, II, III e IV nas abordagens penalizada e hierárquica.

\begin{tabular}{c|cc|cc|cc}
\hline \multirow{2}{*}{ Modelo } & \multicolumn{2}{|c|}{ Função } & \multicolumn{2}{|c|}{ Estimação de $\boldsymbol{\beta}$} & \multicolumn{2}{|c}{ Estimação de $\xi$ ou $\sigma^{2}$} \\
\cline { 2 - 7 } & $\mathbf{P L}$ & HL & PL & HL & PL & HL \\
\hline \multirow{2}{*}{ I } & coxph() & frailtyHL () & $\ell_{P P}$ & $p_{\mathbf{w}}\left(\ell_{P P}\right)$ & $\ell_{m}\left(\lambda_{0}, \boldsymbol{\beta}, \xi\right)$ & $s_{\boldsymbol{\beta}, \mathbf{w}}\left(\ell_{P P}\right)$ \\
\hline II, III e IV & coxme() & frailtyHL () & $\ell_{P P}$ & $p_{\mathbf{w}}\left(\ell_{P P}\right)$ & $p_{\mathbf{w}}\left(\ell_{P P}\right)$ & $p_{\boldsymbol{\beta}, \mathbf{w}}\left(\ell_{P P}\right)$ \\
\hline
\end{tabular}

Pela Tabela 3.5, observamos que os tempos de execução ${ }^{1}$ da função frailtyHL() são muito superiores aos tempos das funções coxph() e coxme(), sendo que o modelo final, por exemplo, levou cerca de 527 vezes mais tempo para ser ajustado na abordagem hierárquica do que na penalizada.

Nesta aplicação, a abordagem penalizada e hierárquica levaram às mesmas conclusões e resultados numéricos muito próximos. No entanto, o algoritmo implementado nas funções coxph() e coxme() é mais eficiente do que o da função frailtyHL(), apresentando menores tempos de execução.

\footnotetext{
${ }^{1} \mathrm{O}$ computador utilizado para estimar os tempos de execução é um Intel Core i5-3330 com CPU de $3.00 \mathrm{GHz} \times 4$ e $8 \mathrm{~GB}$ de RAM.
} 
Tabela 3.5: Tempo de execução de cada modelo, em segundos.

\begin{tabular}{c|lc}
\hline \multirow{2}{*}{ Modelo } & \multicolumn{2}{|c}{ Tempo de execução } \\
& PL & HL \\
\hline I & 0,099 & 112,569 \\
II & 0,259 & 48,067 \\
III & 2,641 & 647,035 \\
IV & 5,058 & 2635,570 \\
\hline
\end{tabular}




\section{Capítulo 4}

\section{Simulação}

O estudo de simulação deste trabalho procurou investigar o viés e a precisão das estimativas dos parâmetros associados aos efeitos fixos e aleatórios de modelos de fragilidade sob a abordagem penalizada e hierárquica. Foram considerados modelos Gama com um componente de fragilidade e modelos Log-normal com dois componentes de fragilidade em cenários próximos ao do discutido no exemplo prático. As simulações foram realizadas no software R.

\subsection{Modelos com um componente de fragilidade}

Neste estudo de simulação, foi considerado o seguinte modelo de fragilidade

$$
\lambda_{i j}\left(t \mid x_{i j}, w_{i}\right)=\lambda_{0}(t) \exp \left\{\beta x_{i j}+w_{i}\right\},
$$

para $i=1, \ldots, k$ e $j=1, \ldots, n_{i}$, em que $\lambda_{i j}(\cdot)$ é a função de risco para o $j$-ésimo tempo pertencente ao $i$-ésimo indivíduo, $x_{i j}$ é uma variável indicadora de grupos, $w_{i}$ é a fragilidade do $i$-ésimo indivíduo e $\beta$ é um parâmetro desconhecido.

Os valores $x_{i j}$ foram geradas a partir de uma distribuição Bernoulli com parâmetro q. Como cada indivíduo pertence a um único grupo em todos os instantes de medição, temos que $x_{i 1}=x_{i 2}=\ldots=x_{i n_{i}}$, em que $n_{i}$ é o número de tempos medidos no $i$-ésimo indivíduo.

Os termos de fragilidade $w_{i}$ foram gerados a partir da distribuição $\operatorname{Gama}(1 / \xi, 1 / \xi)$, sendo que o parâmetro $\xi$ foi controlado a partir do coeficiente de concordância de Kendall (Hougaard, 1987), dado por

$$
\tau=\frac{1}{2+\xi} .
$$


Dessa forma, podemos escrever

$$
\xi=\frac{2 \tau}{1-\tau}
$$

Os tempos de falha $T_{i j}$ foram gerados a partir da distribuição exponencial com parâmetro $\theta_{i j}$. Neste caso, a função de risco é dada por

$$
\lambda_{i j}(t)=\theta_{i j}
$$

e, portanto, passa a ser constante em relação a $t$. Assim, dado o modelo em (4.1.1), temos que

$$
\theta_{i j}=\lambda_{0} \exp \left\{\beta x_{i j}+w_{i}\right\}
$$

em que $\lambda_{0}$ é uma constante.

Assim como na aplicação, vamos considerar censura do Tipo I, isto é, a proporção de censura será dada por

$$
p=P\left(T_{i j}>t^{*}\right)=\exp \left\{-\theta_{i j} t^{*}\right\}=\exp \left\{-\lambda_{0} \exp \left\{\beta x_{i j}+w_{i}\right\} t^{*}\right\} .
$$

Como $p$ depende do termo aleatório $w_{i}$, utilizamos $E(p)$ em vez de $p$ para não gerar censura informativa. Da mesma forma, utilizamos $E\left(X_{i j}\right)=q$ no lugar de $x_{i j}$. O cálculo de $E(p)$ se encontra no Apêndice B. Portanto, podemos escrever $t^{*}$ em função dos valores fixados $p, \lambda_{0} \beta$ e $q$, isto é,

$$
t^{*}=\frac{q^{-\xi}-1}{\lambda_{0} \exp (\beta q) \xi}
$$

e $t_{i j}$ será censurado se $t_{i j}>t^{*}$.

Assim como na aplicação, a estimação foi feita pelas funções coxph() e frailtyHL() para as abordagens penalizada e hierárquica, respectivamente.

\subsubsection{Resultados}

Os cenários considerados nessa simulação foram variações daquele encontrado no estudo do ICB-USP, mais precisamente, nos resultados encontrados para o modelo Gama ajustado no capítulo anterior. Na Tabela 4.1 apresentamos todos os valores fixados para a geração das amostras. Devido ao longo tempo de execução da função frailtyHL(), não foi possível considerar todas as combinações dos cenários. Em todas as simulações, cada cenário foi replicado 1000 vezes. 
Os valores fixados para o Tau de Kendall foram escolhidos visando cenários verossímeis baseados na aplicação. Valores muito maiores que 0,1 implicariam em valores altos para $\xi$ e, por sua vez, implicariam em tempos de falhas muito pequenos ou muito grandes, que não seriam razoáveis nesse estudo.

Tabela 4.1: Valores considerados na simulação com um componente de fragilidade.

\begin{tabular}{c|c|c}
\hline Parâmetros da simulação & Descrição & Valores considerados \\
\hline$n_{i}$ & Número de obs. do indivíduo $i$. & 5,30 e 50 \\
$\lambda_{0}$ & Número de indivíduos. & 10,30 e 50 \\
$\beta$ & Função de risco basal. & 1 \\
$p$ & Parâmetro associado à covariável. & 0,5 \\
$q$ & Proporção de censura. & 0,15 e 0,50 \\
$(\xi)$ & Parâmetro da Bernoulli. & 0,5 \\
\hline
\end{tabular}

A função frailtyHL() apresenta, em sua saída padrão, um indicador de convergência. Quando qualquer um dos algoritmos de estimação não converge dentro do número de iterações fixado, esse indicador aponta não-convergência, mas, mesmo assim, reporta as estimativas encontradas. A função $\operatorname{coxph}()$ não traz informação sobre a convergência do algoritmo, contudo, observando os resultados da simulação, muitas estimativas para $\xi$ foram iguais a 5e-07. Sendo assim, consideramos que, nesses casos, o algoritmo divergiu em direção ao zero e, portanto, não obteve convergência. Dessa maneira, na Tabela 4.2, apresentamos a porcentagem de convergência das 1000 réplicas de cada cenário nas duas abordagens. Como a porcentagem de convergência é próxima a $100 \%$ para os cenários simulados $\operatorname{com} \xi=0,22$ (diferente do que acontece com $\xi=0,02$ ), notamos que a nãoconvergência parece estar ligada à estimação do parâmetro associado ao efeito aleatório. Também observamos que a convergência aumenta para cenários com $k$ e $n_{i}$ conjuntamente altos e com menor proporção de censura. Nesse aspecto, não encontramos indícios de cenários em que uma das metodologias seja melhor que a outra.

Na Tabela 4.3 apresentamos o viés relativo das estimativas de $\beta$ (em relação ao seu verdadeiro valor) encontrado em cada cenário. Observamos que a abordagem penalizada 
apresenta menor viés na maioria dos casos considerados. Em geral, nos dois métodos de estimação, o viés relativo de $\hat{\beta}$ é menor nos cenários com $k$ e $n_{i}$ conjuntamente altos e com menor proporção de censura.

A partir da Tabela 4.4, observamos que a variabilidade das estimativas de $\beta$ é menor nos cenários com $k$ e $n_{i}$ iguais a 30. Como esperado, as maiores variabilidades foram encontradas nos cenários com $n_{i}$ pequeno e maior proporção de censura. Os cenários com $\tau=0.1$ apresentaram variabilidade levemente maior do que aqueles com $\tau=0,01$. Em geral, as duas abordagens apresentaram variabilidades bem próximas para as estimativas de $\beta$, sendo que a penalizada variou menos em todos os cenários com o menor valor de $\tau$ e na maioria dos casos com $\tau=0,1$. Pela Tabela 4.5, verificamos que o erro quadrático médio do $\hat{\beta}$ não difere muito entre as duas abordagens, sendo que, em geral, é menor na penalizada.

Para a análise das estimativas do parâmetro de fragilidade, optamos por retirar todos os casos (das duas abordagens) que não convergiram segundo os critérios estabelecidos aqui. Como muitas dessas simulações conduziram a estimativas muito discrepantes ${ }^{1}$ de $\xi$, que em estudos práticos não seriam considerados, a não-exclusão desses resultados implicaria na superestimação do viés e da variabilidade de $\hat{\xi}$. Sendo assim, pela Tabela 4.6, observamos a abordagem penalizada apresentou melhores resultados para $n_{i}$ pequeno, enquanto que, para $k$ pequeno, a abordagem hierárquica levou a um viés menor. Em geral, para $\tau=0,01$ o viés foi menor com a metodologia penalizada e, para $\tau=0,1$, a metodologia hierárquica levou a estimativas menos enviesadas.

Pela Tabela 4.7, observamos que a variabilidade das estimativas de $\xi$, assim como o erro quadrático médio (Tabela 4.8), sob a estimação hierárquica é menor na maioria dos casos (exceção aos cenários com $n_{i}=5$ e $\tau=0,1$ ). Além disso, observamos que também para $\hat{\xi}$ os cenários com menor variação são aqueles com $k$ e $n_{i}$ conjuntamente altos.

Para ilustrar melhor os resultados da simulação discutidos até aqui, apresentamos no Apêndice C os gráficos das estimativas de $\beta$ (Figuras C.1 a C.8) e $\xi$ (Figuras C.9 a C.16) para as 1000 réplicas de cada cenário considerado. Podemos observar que, em termos de viés e variabilidade, as duas abordagens realmente não apresentam grandes diferenças. Além disso, de fato notamos que os cenários com $n_{i}=30$ e $k=30$ levaram a um menor viés e menor variabilidade nas duas metodologias.

Ainda utilizando o modelo Gama, realizamos um segundo estudo de simulação, ignorando agora o cenário do estudo do ICB-USP, com o intuito de ilustrar o problema de finite sample bias na estimação de $\xi$ pela abordagem penalizada, como discutido na

\footnotetext{
${ }^{1}$ Obtivemos tanto valores que divergiram em direção do zero (grande maioria) quanto na direção de $+\infty$.
} 
seção 2.1.1. Simulamos cenários com dados não-censurados, $\tau=0,1$ e $k$ e $n_{i}$ variando, respectivamente, entre (3 e 10) e (20, 50 e 100). Observe que algumas dessas combinações dificilmente são encontrados em casos práticos e, por conta disso, fizemos esse estudo separadamente. Podemos observar pelos gráficos apresentados nas Figuras 4.1 e 4.2 que, na abordagem penalizada, grande parte das estimativas de $\xi$ passam a ser altamente enviesadas conforme $n_{i}$ cresce, o que não acontece na abordagem hierárquica. A escolha por cenários não-censurados se deve ao fato de que esse viés diminui com o aumento da censura. Essa relação pode parecer contra-intuitiva, mas a perda de informação causada pela censura diminui o número de parâmetros de perturbação a ser estimado, levando, então, à diminuição do viés.

Tabela 4.2: Porcentagem de convergência para cada cenário simulado.

\begin{tabular}{ccc|cc||cc}
\hline \hline \multirow{2}{*}{$\mathbf{k}$} & \multirow{2}{*}{$\mathbf{n}_{\mathbf{i}}$} & \multirow{2}{*}{ Abordagem } & \multicolumn{3}{|c}{ Convergência (\%) } \\
\cline { 4 - 7 } & & & \multicolumn{2}{|c}{$\boldsymbol{\tau}=\mathbf{0 , 0 1}$} & \multicolumn{2}{c}{$\boldsymbol{\tau}=\mathbf{0 , 1}$} \\
\cline { 4 - 7 } & & & $\mathrm{p}=0,15$ & $\mathrm{p}=0,5$ & $\mathrm{p}=0,15$ & $\mathrm{p}=0,5$ \\
\hline $\mathbf{1 0}$ & $\mathbf{3 0}$ & $\mathbf{P L}$ & 69,07 & 55,20 & 99,90 & 98,50 \\
$\mathbf{1 0}$ & $\mathbf{3 0}$ & $\mathbf{H L}$ & 63,66 & 70,90 & 99,80 & 98,90 \\
\hline $\mathbf{1 0}$ & $\mathbf{5 0}$ & $\mathbf{P L}$ & 77,05 & 64,50 & 100,00 & 99,70 \\
$\mathbf{1 0}$ & $\mathbf{5 0}$ & $\mathbf{H L}$ & 37,98 & 57,70 & 99,70 & 99,60 \\
\hline $\mathbf{3 0}$ & $\mathbf{5}$ & $\mathbf{P L}$ & 52,40 & 49,60 & 96,80 & 91,30 \\
$\mathbf{3 0}$ & $\mathbf{5}$ & $\mathbf{H L}$ & 68,60 & 68,40 & 96,30 & 88,50 \\
\hline $\mathbf{3 0}$ & $\mathbf{3 0}$ & $\mathbf{P L}$ & 90,79 & 77,60 & 100,00 & 100,00 \\
$\mathbf{3 0}$ & $\mathbf{3 0}$ & $\mathbf{H L}$ & 78,38 & 81,10 & 100,00 & 100,00 \\
\hline $\mathbf{5 0}$ & $\mathbf{5}$ & $\mathbf{P L}$ & 57,50 & 54,00 & 99,70 & 96,30 \\
$\mathbf{5 0}$ & $\mathbf{5}$ & $\mathbf{H L}$ & 60,90 & 59,50 & 98,90 & 92,50 \\
\hline \hline
\end{tabular}


Tabela 4.3: Viés relativo das estimativas de $\beta$ para cada cenário.

\begin{tabular}{|c|c|c|c|c|c|c|c|}
\hline \multicolumn{8}{|c|}{ Viés relativo $-\beta$} \\
\hline \multirow{3}{*}{$\mathrm{k}$} & \multirow{3}{*}{ Abordagem } & \multicolumn{6}{|c|}{$\tau=0,01$} \\
\hline & & \multicolumn{3}{|c|}{$\mathrm{p}=0,15$} & \multicolumn{3}{|c|}{$\mathrm{p}=0,50$} \\
\hline & & $\mathbf{n}_{\mathbf{i}}=5$ & $\mathbf{n}_{\mathbf{i}}=30$ & $\mathbf{n}_{\mathbf{i}}=50$ & $\mathrm{n}_{\mathrm{i}}=5$ & $\mathrm{n}_{\mathrm{i}}=30$ & $\mathbf{n}_{\mathbf{i}}=50$ \\
\hline 10 & PL & - & $-0,036 \%$ & $0,812 \%$ & - & $-0,680 \%$ & $0,926 \%$ \\
\hline 10 & HL & - & $1,076 \%$ & $1,714 \%$ & - & $-0,312 \%$ & $1,356 \%$ \\
\hline 30 & PL & $-0,092 \%$ & $0,308 \%$ & - & $2,328 \%$ & $0,626 \%$ & - \\
\hline 30 & HL & $0,806 \%$ & $0,910 \%$ & - & $2,898 \%$ & $0,756 \%$ & - \\
\hline 50 & PL & $1,830 \%$ & - & - & $2,122 \%$ & - & - \\
\hline \multirow[t]{2}{*}{50} & HL & $2,476 \%$ & - & - & $2,534 \%$ & - & - \\
\hline & & \multicolumn{6}{|c|}{$\tau=0,1$} \\
\hline 10 & PL & - & $0,002 \%$ & $0,970 \%$ & - & $1,994 \%$ & $0,016 \%$ \\
\hline 10 & HL & - & $-0,094 \%$ & $0,556 \%$ & - & $2,142 \%$ & $-0,092 \%$ \\
\hline 30 & PL & $-0,734 \%$ & $0,546 \%$ & - & $0,748 \%$ & $-0,036 \%$ & - \\
\hline 30 & HL & $0,748 \%$ & $0,136 \%$ & - & $1,600 \%$ & $-0,044 \%$ & - \\
\hline 50 & PL & $0,966 \%$ & - & - & $1,938 \%$ & - & - \\
\hline 50 & HL & $2,156 \%$ & - & - & $2,636 \%$ & - & - \\
\hline
\end{tabular}


Tabela 4.4: Variância média das estimativas de $\beta$ para cada cenário.

\begin{tabular}{|c|c|c|c|c|c|c|c|}
\hline \multicolumn{8}{|c|}{ Variância das estimativas de $\beta$} \\
\hline \multirow{3}{*}{$\mathrm{k}$} & \multirow{3}{*}{ Abordagem } & \multicolumn{6}{|c|}{$\tau=0,01$} \\
\hline & & \multicolumn{3}{|c|}{$\mathrm{p}=\mathbf{0}, \mathbf{1 5}$} & \multicolumn{3}{|c|}{$\mathrm{p}=0,50$} \\
\hline & & $\mathrm{n}_{\mathrm{i}}=\mathbf{5}$ & $\mathbf{n}_{\mathbf{i}}=30$ & $\mathbf{n}_{\mathrm{i}}=50$ & $\mathbf{n}_{\mathrm{i}}=5$ & $\mathbf{n}_{\mathbf{i}}=30$ & $\mathbf{n}_{\mathbf{i}}=50$ \\
\hline 10 & PL & - & 0,01701 & 0,00862 & - & 0,02677 & 0,01716 \\
\hline 10 & HL & - & 0,01706 & 0,00863 & - & 0,02696 & 0,01723 \\
\hline 30 & PL & 0,03411 & 0,00566 & - & 0,06091 & 0,00913 & - \\
\hline 30 & HL & 0,03505 & 0,00566 & - & 0,06223 & 0,00915 & - \\
\hline 50 & PL & 0,02121 & - & - & 0,03824 & - & - \\
\hline \multirow[t]{2}{*}{50} & HL & 0,02178 & - & - & 0,03890 & - & - \\
\hline & & \multicolumn{6}{|c|}{$\tau=0,1$} \\
\hline 10 & PL & - & 0,01749 & 0,00933 & - & 0,03047 & 0,01716 \\
\hline 10 & HL & - & 0,01742 & 0,00925 & - & 0,03048 & 0,01712 \\
\hline 30 & PL & 0,03950 & 0,00547 & - & 0,05908 & 0,00998 & - \\
\hline 30 & HL & 0,04048 & 0,00540 & - & 0,06030 & 0,00998 & - \\
\hline 50 & PL & 0,02420 & - & - & 0,03998 & - & - \\
\hline 50 & HL & 0,02466 & - & - & 0,04045 & - & - \\
\hline
\end{tabular}


Tabela 4.5: Erro quadrático médio das estimativas de $\beta$ para cada cenário.

\begin{tabular}{|c|c|c|c|c|c|c|c|}
\hline \multicolumn{8}{|c|}{ Erro quadrático médio $-\beta$} \\
\hline \multirow{3}{*}{ k } & \multirow{3}{*}{ Abordagem } & \multicolumn{6}{|c|}{$\tau=0,01$} \\
\hline & & \multicolumn{3}{|c|}{$\mathrm{p}=0,15$} & \multicolumn{3}{|c|}{$\mathrm{p}=0,50$} \\
\hline & & $\mathrm{n}_{\mathrm{i}}=5$ & $\mathbf{n}_{\mathbf{i}}=30$ & $\mathbf{n}_{\mathbf{i}}=50$ & $\mathbf{n}_{\mathbf{i}}=\mathbf{5}$ & $\mathbf{n}_{\mathbf{i}}=30$ & $\mathbf{n}_{\mathbf{i}}=50$ \\
\hline 10 & PL & - & 0,01702 & 0,00866 & - & 0,02682 & 0,01721 \\
\hline 10 & HL & - & 0,01707 & 0,00868 & - & 0,02699 & 0,01728 \\
\hline 30 & PL & 0,03419 & 0,00567 & - & 0,06169 & 0,00916 & - \\
\hline 30 & HL & 0,03535 & 0,00567 & - & 0,06338 & 0,00918 & - \\
\hline 50 & PL & 0,02139 & - & - & 0,03872 & - & - \\
\hline \multirow[t]{2}{*}{50} & HL & 0,02214 & - & - & 0,03957 & - & - \\
\hline & & \multicolumn{6}{|c|}{$\tau=0,1$} \\
\hline 10 & PL & - & 0,01749 & 0,00935 & - & 0,03057 & 0,01716 \\
\hline 10 & HL & - & 0,01742 & 0,00925 & - & 0,03060 & 0,01712 \\
\hline 30 & PL & 0,03950 & 0,00548 & - & 0,05915 & 0,00998 & - \\
\hline 30 & HL & 0,04051 & 0,00540 & - & 0,06046 & 0,00998 & - \\
\hline 50 & PL & 0,02423 & - & - & 0,04019 & - & - \\
\hline 50 & HL & 0,02479 & - & - & 0,04078 & - & - \\
\hline
\end{tabular}


Tabela 4.6: Viés relativo das estimativas de $\xi$ para cada cenário.

\begin{tabular}{|c|c|c|c|c|c|c|c|}
\hline & \multicolumn{7}{|c|}{ Viés - $\xi$} \\
\hline \multirow{3}{*}{$\mathbf{k}$} & \multirow{3}{*}{ Abordagem } & \multicolumn{6}{|c|}{$\tau=0,01$} \\
\hline & & \multicolumn{3}{|c|}{$\mathrm{p}=0,15$} & \multicolumn{3}{|c|}{$\mathrm{p}=0,50$} \\
\hline & & $\mathrm{n}_{\mathrm{i}}=5$ & $\mathbf{n}_{\mathbf{i}}=30$ & $\mathrm{n}_{\mathrm{i}}=50$ & $\mathbf{n}_{\mathbf{i}}=5$ & $\mathbf{n}_{\mathbf{i}}=30$ & $\mathrm{n}_{\mathrm{i}}=50$ \\
\hline 10 & PL & - & $288,60 \%$ & $95,75 \%$ & - & $173,85 \%$ & $108,85 \%$ \\
\hline 10 & HL & - & $85,6 \%$ & $106,45 \%$ & - & $139,65 \%$ & $87,05 \%$ \\
\hline 30 & PL & $233,2 \%$ & $19,75 \%$ & - & $492,45 \%$ & $39,7 \%$ & - \\
\hline 30 & HL & $370,3 \%$ & $30,2 \%$ & - & $692,6 \%$ & $58,85 \%$ & - \\
\hline 50 & PL & $203,5 \%$ & - & - & $203,5 \%$ & - & - \\
\hline \multirow[t]{2}{*}{50} & HL & $303,90 \%$ & - & - & $546,45 \%$ & - & - \\
\hline & & \multicolumn{6}{|c|}{$\tau=0,1$} \\
\hline 10 & PL & - & $12,20 \%$ & $66,82 \%$ & - & $-3,41 \%$ & $17,42 \%$ \\
\hline 10 & HL & - & $-2,14 \%$ & $1,58 \%$ & - & $2,74 \%$ & $1,01 \%$ \\
\hline 30 & PL & $-7,50 \%$ & $25,43 \%$ & - & $13,07 \%$ & $2,49 \%$ & - \\
\hline 30 & HL & $8,45 \%$ & $-0,80 \%$ & - & $30,76 \%$ & $1,22 \%$ & - \\
\hline 50 & PL & $-7,44 \%$ & - & - & $3,46 \%$ & - & - \\
\hline 50 & HL & $5,15 \%$ & - & - & $15,52 \%$ & - & - \\
\hline
\end{tabular}


Tabela 4.7: Variância média das estimativas de $\xi$ para cada cenário.

\begin{tabular}{|c|c|c|c|c|c|c|c|}
\hline \multicolumn{8}{|c|}{ Variância das estimativas de $\xi$} \\
\hline \multirow{3}{*}{$\mathrm{k}$} & \multirow{3}{*}{ Abordagem } & \multicolumn{6}{|c|}{$\tau=0,01$} \\
\hline & & \multicolumn{3}{|c|}{$\mathrm{p}=0,15$} & \multicolumn{3}{|c|}{$\mathrm{p}=0,50$} \\
\hline & & $\mathbf{n}_{\mathbf{i}}=5$ & $\mathbf{n}_{\mathbf{i}}=30$ & $\mathbf{n}_{\mathbf{i}}=50$ & $\mathrm{n}_{\mathrm{i}}=\mathbf{5}$ & $\mathbf{n}_{\mathbf{i}}=\mathbf{3 0}$ & $\mathbf{n}_{\mathbf{i}}=50$ \\
\hline 10 & PL & - & 0,17161 & 0,0004 & - & 0,04116 & 0,04731 \\
\hline 10 & HL & - & 0,00054 & 0,0003 & - & 0,00114 & 0,00054 \\
\hline 30 & PL & 0,00255 & 0,00019 & - & 0,00857 & 0,00046 & - \\
\hline 30 & HL & 0,00253 & 0,00018 & - & 0,00773 & 0,00037 & - \\
\hline 50 & PL & 0,00187 & - & - & 0,00483 & - & - \\
\hline \multirow[t]{2}{*}{50} & HL & 0,00158 & - & - & 0,00375 & - & - \\
\hline & & \multicolumn{6}{|c|}{$\tau=0,1$} \\
\hline 10 & PL & - & 0,02774 & 0,04071 & - & 0,01986 & 0,02803 \\
\hline 10 & HL & - & 0,01465 & 0,01263 & - & 0,01764 & 0,01458 \\
\hline 30 & PL & 0,01453 & 0,01166 & - & 0,02485 & 0,00758 & - \\
\hline 30 & HL & 0,01522 & 0,00448 & - & 0,02587 & 0,00574 & - \\
\hline 50 & PL & 0,00908 & - & - & 0,01732 & - & - \\
\hline 50 & HL & 0,00926 & - & - & 0,01662 & - & - \\
\hline
\end{tabular}


Tabela 4.8: Erro quadrático médio das estimativas de $\xi$ para cada cenário.

\begin{tabular}{|c|c|c|c|c|c|c|c|}
\hline \multicolumn{8}{|c|}{ Erro quadrático médio $-\beta$} \\
\hline \multirow{3}{*}{$\mathrm{k}$} & \multirow{3}{*}{ Abordagem } & \multicolumn{6}{|c|}{$\tau=0,01$} \\
\hline & & \multicolumn{3}{|c|}{$\mathrm{p}=0,15$} & \multicolumn{3}{|c|}{$\mathrm{p}=\mathbf{0}, 50$} \\
\hline & & $\mathbf{n}_{\mathbf{i}}=5$ & $\mathbf{n}_{\mathbf{i}}=30$ & $\mathrm{n}_{\mathrm{i}}=50$ & $\mathbf{n}_{\mathrm{i}}=5$ & $\mathbf{n}_{\mathbf{i}}=30$ & $\mathrm{n}_{\mathrm{i}}=50$ \\
\hline 10 & PL & - & 0,1794 & 0,00076 & - & 0,04236 & 0,04778 \\
\hline 10 & HL & - & 0,0083 & 0,00075 & - & 0,02692 & 0,00051 \\
\hline 30 & PL & 0,00472 & 0,00020 & - & 0,01827 & 0,00053 & - \\
\hline 30 & HL & 0,00801 & 0,00022 & - & 0,02692 & 0,00051 & - \\
\hline 50 & PL & 0,00353 & - & - & 0,01113 & - & - \\
\hline \multirow[t]{2}{*}{50} & HL & 0,00527 & - & - & 0,01569 & - & - \\
\hline & & \multicolumn{6}{|c|}{$\tau=0,1$} \\
\hline 10 & PL & - & 0,02816 & 0,06232 & - & 0,01991 & 0,0295 \\
\hline 10 & HL & - & 0,01468 & 0,01264 & - & 0,01768 & 0,01459 \\
\hline 30 & PL & 0,01481 & 0,01479 & - & 0,02567 & 0,00761 & - \\
\hline 30 & HL & 0,01556 & 0,00449 & - & 0,03045 & 0,00575 & - \\
\hline 50 & PL & 0,00935 & - & - & 0,01738 & - & - \\
\hline 50 & HL & 0,00939 & - & - & 0,01779 & - & - \\
\hline
\end{tabular}




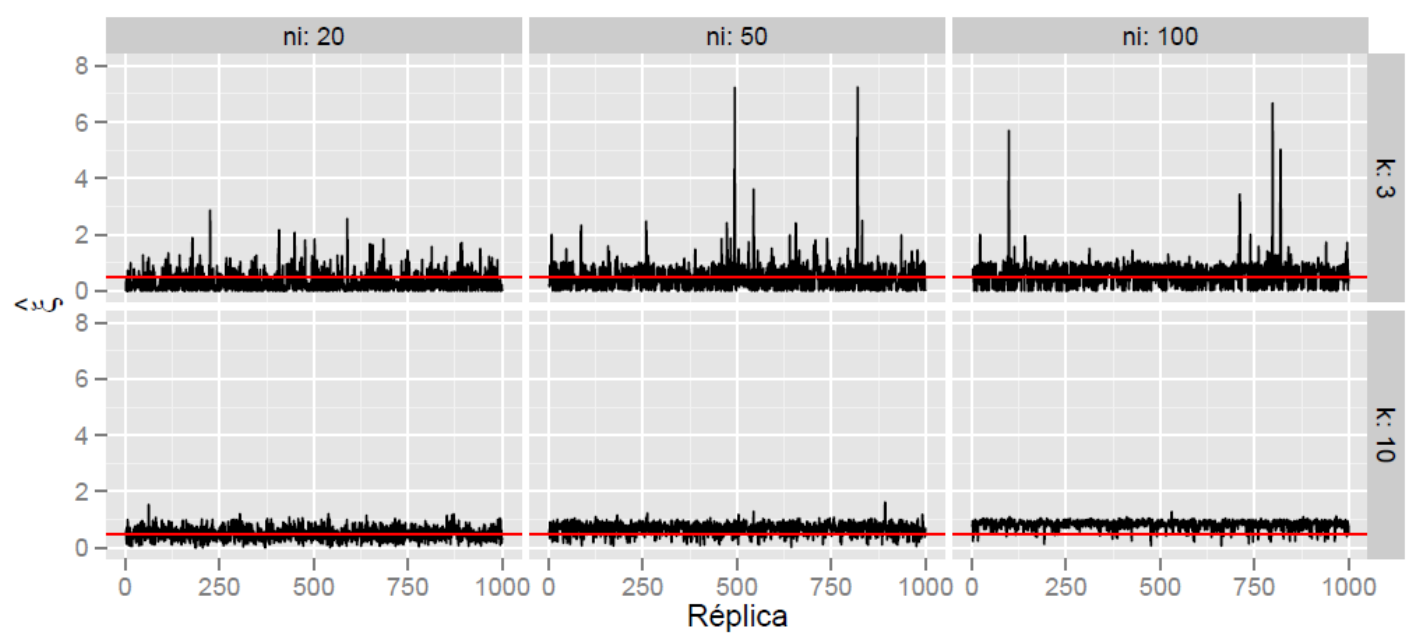

Figura 4.1: Estimativas de $\xi$ da abordagem penalizada do segundo estudo de simulação. Cenários com dados não-censurados e $\tau=0,1$.

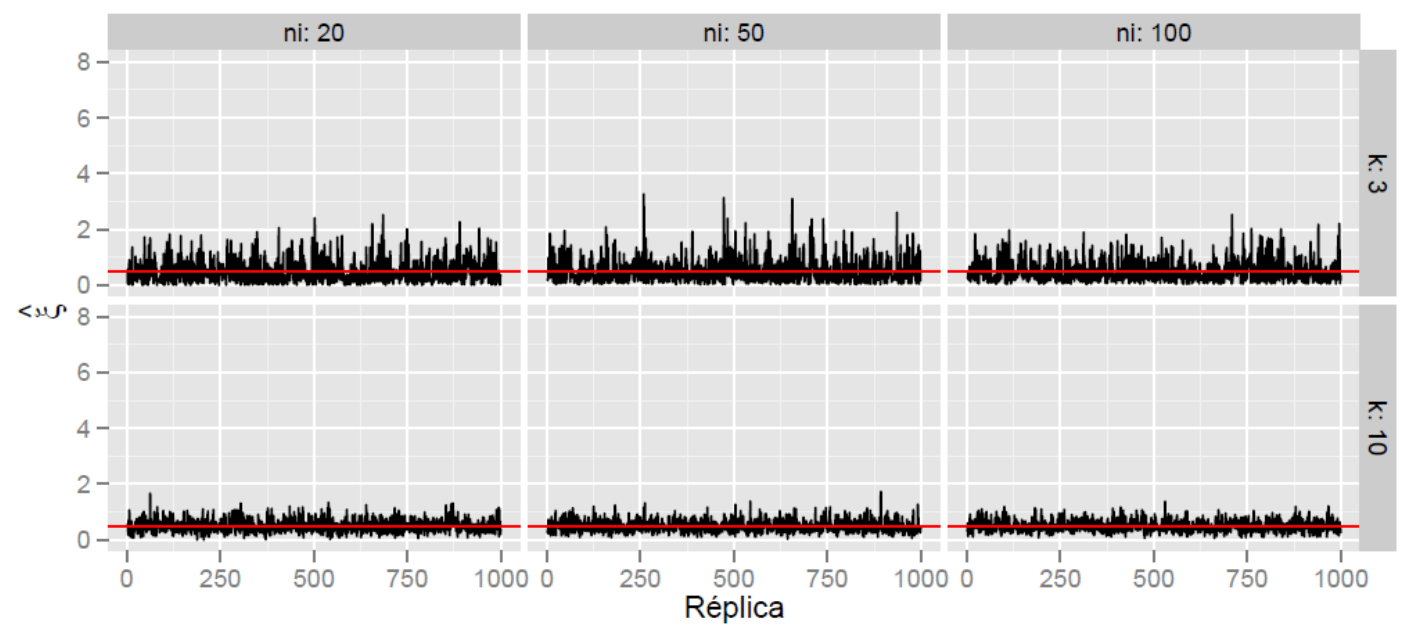

Figura 4.2: Estimativas de $\xi$ da abordagem hierárquica do segundo estudo de simulação. Cenários dados não-censurados e $\tau=0,1$. 


\subsection{Modelos com dois componentes de fragilidade}

Para cenários com dois componentes de fragilidade, foi considerado o seguinte modelo

$$
\lambda_{i j l}\left(t \mid x_{i j l}, w_{i}, v_{i j}\right)=\lambda_{0}(t) \exp \left\{\beta x_{i j l}+w_{i}+v_{i j}\right\} .
$$

Baseado no estudo do ICB-USP, para $i=1, \ldots, k, j=1, \ldots, m_{i}$ e $l=1, \ldots, n_{i j}, \lambda_{i j k}(\cdot)$ representa a função de risco para a $l$-ésima tentativa do $j$-ésimo dia do $i$-ésimo rato, $\beta$ é um parâmetro desconhecido associado a covariável separadora de grupos $x_{i j l}, w_{i}$ é o componente de fragilidade associado à $i$-ésima cobaia e $v_{i j}$ é o componente de fragilidade associado às medidas do $j$-ésimo dia do $i$-ésimo rato.

Os valores $x_{i j l}$ foram gerados a partir de uma distribuição Bernoulli com parâmetro q. Já os termos de fragilidade $w_{i}$ e $v_{i j}$ foram gerados a partir das distribuições $\mathrm{N}\left(0, \sigma_{r}^{2}\right)$ e $\mathrm{N}\left(0, \sigma_{d}^{2}\right)$, respectivamente. Esses componentes podem ser controlados pelas correlações $\rho_{r}$ e $\rho_{d}$ dadas por

$$
\rho_{r}=\operatorname{Corr}\left(T_{i j l}, T_{i j^{\prime} l^{\prime}}\right)=\frac{\exp \left\{\sigma_{r}^{2}\right\}-1}{2 \exp \left\{\sigma_{r}^{2}\right\} \exp \left\{\sigma_{d}^{2}\right\}-1}, \quad \text { para } \quad j \neq j^{\prime}
$$

$\mathrm{e}$

$$
\rho_{d}=\operatorname{Corr}\left(T_{i j l}, T_{i j l^{\prime}}\right)=\frac{\exp \left\{\sigma_{r}^{2}\right\} \exp \left\{\sigma_{d}^{2}\right\}-1}{2 \exp \left\{\sigma_{r}^{2}\right\} \exp \left\{\sigma_{d}^{2}\right\}-1}, \quad \text { para } \quad l \neq l^{\prime} .
$$

Os cálculos dessas expressões se encontram no Apêndice B.

Assim como feito na seção anterior, utilizamos a distribuição exponencial para gerar os tempos de falha $T_{i j l}$. Considerando novamente censura do Tipo I, aqui, temos

$$
p=P\left(T_{i j}>t^{*}\right)=\exp \left\{-\theta_{i j} t^{*}\right\}=\exp \left\{-\lambda_{0} \exp \left\{\beta x_{i j}+w_{i}+v_{i j}\right\} t^{*}\right\} .
$$

Devido aos dois componentes aleatórios, o cálculo de $E(p)$ não é trivial. Portanto, optamos por substituir $w_{i}$ e $v_{i j}$ pelos seus valores médios, assim como foi feito com a covariável $x_{i j}$. Portanto, temos

$$
p=\exp \left\{-\lambda_{0} \exp \{\beta q\} t^{*}\right\}
$$

e, então,

$$
t^{*}=-\frac{\log p}{\lambda_{0} \exp (\beta q)} .
$$

A estimação desses dados foi feita pelas funções coxme() e frailtyHL() para as abordagens penalizada e hierárquica, respectivamente. 


\subsubsection{Resultados}

Os cenários considerados nessa simulação foram baseados nos resultados encontrados para o Modelo III ajustado no capítulo 3. Na Tabela 4.9 apresentamos todos os valores fixados para a geração das amostras. O alto tempo de execução da função frailtyHL() restringiu muito o número de variações dos parâmetros da simulação, assim como a escolha de valores altos para $n_{i j}$ e $m_{i}$. Como apresentado na Tabela 3.5, essa função levou cerca de 10 minutos para ajustar o Modelo III, cujo valor de $n_{i j}$ é 2. Para amostras simuladas com $n_{i j}$ igual a 5 , mantendo todos os outros parâmetros iguais aos do Modelo III, a frailtyHL() apresentou tempos de execução maiores que 1 hora. Considerando as 1000 réplicas, levaríamos mais de 40 dias para obter os resultados de um cenário. Sendo assim, nos cenários desta simulação, optamos por diminuir o número de dias, fixando $m_{i}$ igual a 7, e variar apenas o número de observações de cada rato em cada dia $\left(n_{i j}\right)$ e a proporção de censura $(p)$.

Tabela 4.9: Valores considerados na simulação com dois componente de fragilidade.

\begin{tabular}{c|c|c}
\hline Parâmetros da simulação & Descrição & Valores considerados \\
\hline$n_{i j}$ & Número de obs. do rato $i$. & 2 e 5 \\
$k$ & Número de dias. & 7 \\
$\lambda_{0}$ & Número de ratos. & 32 \\
$\beta$ & Função de risco basal. & 1 \\
$p$ & Parâmetro associado à covariável. & 0,5 \\
$\rho_{r}\left(\sigma_{r}^{2}\right)$ & Proporção de censura. & 0,15 e 0,50 \\
$\rho_{d}\left(\sigma_{d}^{2}\right)$ & Correlação entre medidas & 0,5 \\
de um mesmo rato. & $0,07(0,11)$ \\
\hline
\end{tabular}

Na Tabela 4.10, apresentamos a porcentagem de convergência das 1000 réplicas de cada cenário segundo a função frailtyHL(). A função coxme() não traz informação 
sobre a convergência do algoritmo e, observando os resultados, não encontramos indícios de não-convergência, ao contrário do que foi feito na simulação com o modelo Gama.

Observando a Tabela 4.11, notamos que o viés das estimativas de $\beta$ foi menor na abordagem penalizada para os cenários com $p=0,15$. Já para $p=0,50$, essa situação se inverteu e a abordagem hierárquica passou a apresentar menor viés. Apesar disso, a variabilidade das estimativas foi menor na metodologia penalizada em todos os cenários, o que conduziu, também, a um menor erro quadrático médio.

Analisando agora o parâmetro $\sigma_{r}^{2}$ pela Tabela 4.12, observamos que a abordagem penalizada apresentou viés relativo menores que à abordagem hierárquica em todos os cenários considerados. A respeito da variabilidade e erro quadrático médio, as duas metodologias apresentaram valores bem próximos, sendo que a penalizada levou a resultados ligeiramente melhores.

Por fim, analisando a Tabela 4.13, notamos que o viés relativo das estimativas de $\sigma_{d}^{2}$ é menor na abordagem hierárquica em todos os casos. Mais uma vez, as duas metodologias apresentaram valores bem próximos para a variabilidade e erro quadrático médio, sendo que a abordagem hierárquica levou a resultados ligeiramente melhores.

No Apêndice III, apresentamos os gráficos para as estimativas de $\beta$, ao longo das 1000 réplicas, referentes aos cenários considerados (Figuras C.17 e C.18), assim como os gráficos para as estimativas de $\sigma_{r}^{2}$ e $\sigma_{d}^{2}$ (Figuras C.19 a C.22).

Tabela 4.10: Porcentagem de convergência para cada cenário simulado da abordagem hierárquica.

\begin{tabular}{cc|cc}
\hline & & \multicolumn{2}{|c}{ Convergência } \\
$\mathbf{n i}$ & Abordagem & $\mathbf{p}=\mathbf{0 , 1 5}$ & $\mathbf{p}=\mathbf{0 , 5 0}$ \\
\hline $\mathbf{2}$ & $\mathbf{P L}$ & - & - \\
$\mathbf{2}$ & HL & 73,4 & 58,8 \\
\hline $\mathbf{5}$ & PL & - & - \\
$\mathbf{5}$ & HL & 99,9 & 98,7 \\
\hline
\end{tabular}


Tabela 4.11: Viés relativo, variância e erro quadrático médio das estimativas de $\beta$ para cada cenário.

\begin{tabular}{|c|c|c|c|}
\hline \multicolumn{4}{|c|}{ Viés - $\hat{\boldsymbol{\beta}}$} \\
\hline ni & Abordagem & $\mathrm{p}=0,15$ & $\mathrm{p}=0,50$ \\
\hline 2 & PL & $0,106 \%$ & $-1,608 \%$ \\
\hline 2 & HL & $0,958 \%$ & $-0,798 \%$ \\
\hline 5 & PL & $0,500 \%$ & $-0,440 \%$ \\
\hline 5 & HL & $0,770 \%$ & $-0,146 \%$ \\
\hline \multicolumn{4}{|c|}{$\operatorname{Var}-\hat{\boldsymbol{\beta}}$} \\
\hline 2 & PL & 0,01467 & 0,02160 \\
\hline 2 & HL & 0,01500 & 0,02200 \\
\hline 5 & PL & 0,00525 & 0,00785 \\
\hline 5 & HL & 0,00528 & 0,00790 \\
\hline \multicolumn{4}{|c|}{$\mathrm{EQM}-\hat{\boldsymbol{\beta}}$} \\
\hline 2 & PL & 0,01467 & 0,02166 \\
\hline 2 & HL & 0,01502 & 0,02201 \\
\hline 5 & PL & 0,00526 & 0,00785 \\
\hline 5 & HL & 0,00530 & 0,00790 \\
\hline
\end{tabular}


Tabela 4.12: Viés relativo, variância e erro quadrático médio das estimativas de $\sigma_{r}^{2}$ para cada cenário.

\begin{tabular}{|c|c|c|c|}
\hline \multicolumn{4}{|c|}{ Viés $-\hat{\sigma}_{r}^{2}$} \\
\hline ni & Abordagem & $\mathrm{p}=0,15$ & $\mathrm{p}=0,50$ \\
\hline 2 & PL & $0,209 \%$ & $0,645 \%$ \\
\hline 2 & HL & $0,691 \%$ & $1,027 \%$ \\
\hline 5 & PL & $0,845 \%$ & $3,372 \%$ \\
\hline 5 & HL & $0,927 \%$ & $3,427 \%$ \\
\hline \multicolumn{4}{|c|}{$\operatorname{Var}-\hat{\sigma}_{r}^{2}$} \\
\hline 2 & PL & 0,00409 & 0,00520 \\
\hline 2 & HL & 0,00412 & 0,00524 \\
\hline 5 & PL & 0,00204 & 0,00279 \\
\hline 5 & HL & 0,00204 & 0,00279 \\
\hline \multicolumn{4}{|c|}{$\mathrm{EQM}-\hat{\sigma}_{r}^{2}$} \\
\hline 2 & PL & 0,00409 & 0,00520 \\
\hline 2 & HL & 0,00412 & 0,00524 \\
\hline 5 & PL & 0,00204 & 0,00280 \\
\hline 5 & HL & 0,00204 & 0,00280 \\
\hline
\end{tabular}


Tabela 4.13: Viés relativo, variância e erro quadrático médio das estimativas de $\sigma_{d}^{2}$ para cada cenário.

\begin{tabular}{|c|c|c|c|}
\hline \multicolumn{4}{|c|}{ Viés $-\hat{\sigma}_{d}^{2}$} \\
\hline ni & Abordagem & $\mathrm{p}=0,15$ & $\mathrm{p}=0,50$ \\
\hline 2 & PL & $9,606 \%$ & $11,928 \%$ \\
\hline 2 & HL & $7,089 \%$ & $9,189 \%$ \\
\hline 5 & PL & $2,867 \%$ & $5,906 \%$ \\
\hline 5 & HL & $2,572 \%$ & $5,578 \%$ \\
\hline \multicolumn{4}{|c|}{$\operatorname{Var}-\hat{\sigma}_{d}^{2}$} \\
\hline 2 & PL & 0,01106 & 0,01474 \\
\hline 2 & HL & 0,01115 & 0,01492 \\
\hline 5 & PL & 0,00251 & 0,00412 \\
\hline 5 & HL & 0,00251 & 0,00412 \\
\hline \multicolumn{4}{|c|}{$\mathrm{EQM}-\hat{\sigma}_{d}^{2}$} \\
\hline 2 & PL & 0,01136 & 0,01520 \\
\hline 2 & HL & 0,01131 & 0,01519 \\
\hline 5 & PL & 0,00254 & 0,00423 \\
\hline 5 & HL & 0,00254 & 0,00422 \\
\hline
\end{tabular}




\section{Capítulo 5}

\section{Conclusões}

Este trabalho mostrou que a utilização da abordagem penalizada ou hierárquica no processo de estimação de modelos de fragilidade, na maioria dos casos, conduz a resultados numéricos muito próximos, tanto em termos do viés e da variabilidade das estimativas quanto na avaliação nos resultados inferenciais, como foi ilustrado pela análise do estudo do ICB-USP.

Pelo estudo de simulação com um componente de fragilidade, observamos que a estimação por meio da função $\operatorname{coxph}()$ para o modelo Gama pode levar à estimativas enviesadas para o parâmetro de fragilidade em cenários com amostras muito grandes, o que não acontece na metodologia hierárquica. No entanto, para os parâmetros associados aos efeitos fixos, a abordagem penalizada levou a resultados ligeiramente melhores na maioria dos casos.

Para as simulações com dois componentes de fragilidade, encontramos indícios de que a abordagem hierárquica conduz a estimativas com menor viés em cenários com tamanho de amostra pequeno ou alta proporção de censura. Nos demais casos, a metodologia penalizada apresentou melhores resultados.

Em todos os modelos considerados neste estudo, as funções $\operatorname{coxph}()$ e coxme() foram sempre mais eficientes que a função frailtyHL(), sendo que a diferença entre os tempos de execução em alguns casos chegou a ser maior que 1 hora. Do ponto de vista prático, essa foi a maior discrepância encontrada entre as duas metodologias, pois o alto tempo de execução do algoritmo disponível para a abordagem hierárquica pode tornar o seu uso inviável em situações em que vários modelos precisam ser ajustados sequencialmente.

Para completar a avaliação feita neste estudo, a correlação entre os componentes de fragilidade deve ser considerada em trabalhos futuros. Além disso, pesquisas direcionadas ao desenvolvimento de técnicas de diagnóstico para modelos de fragilidade Log-normal 
e a implementação de algoritmos mais eficientes para a metodologia hierárquica seriam grandes contribuições dentro deste contexto. 


\section{Apêndice A}

\section{Expressões do Capítulo 2}

Neste apêndice, desenvolvemos algumas expressões apresentadas no Capítulo 2.

\section{Desenvolvimento da expressão (2.2.5)}

A função de verossimilhança restrita, derivada por Patterson e Thompson (1971) e Harville (1977), é muito utilizada na estimação de componentes de variância por conduzir a estimativas não-enviesadas para esses termos e não envolver parâmetros associados aos efeitos fixos ou aleatórios. Como em muitos casos não é possível encontrar uma expressão analítica para a verossimilhança restrita, Cox e Reid (1987) sugeriram a utilização de uma verossimilhança ajustada derivada a partir do método de Laplace para a aproximação de integrais. No contexto de modelos de fragilidade, essa aproximação é dada por

$$
\exp \left(\ell_{R}\right)=\int \exp \left\{h^{*}(\boldsymbol{\alpha})\right\} d \boldsymbol{\alpha} \approx 2 \pi \operatorname{det}\left\{J\left(h^{*}, \hat{\boldsymbol{\alpha}}\right)\right\}^{-\frac{1}{2}} \exp \left\{h^{*}(\hat{\boldsymbol{\alpha}})\right\}=\exp \left(h_{A}\right),
$$

em que $\ell_{R}$ é a log-verossimilhança restrita para $\xi, \boldsymbol{\alpha}=(\boldsymbol{\beta}, \mathbf{w}), \hat{\boldsymbol{\alpha}}=(\hat{\boldsymbol{\beta}}, \hat{\mathbf{w}})$ resolve a equação escore $\partial h^{*} / \partial \alpha=0$ e $J(\cdot)$ é a matriz de informação observada dada por (2.2.4). Dessa forma, temos

$$
\begin{aligned}
h_{A} & =\log \left(2 \pi \operatorname{det}\{J(\hat{\boldsymbol{\alpha}})\}^{-\frac{1}{2}} \exp \left\{h^{*}(\hat{\boldsymbol{\alpha}})\right\}\right) \\
& =h^{*}(\hat{\boldsymbol{\alpha}})-\frac{1}{2} \log \left(\frac{\operatorname{det}\{J(\hat{\boldsymbol{\alpha}})\}}{4 \pi^{2}}\right) \\
& =h^{*}(\hat{\boldsymbol{\alpha}})-\frac{1}{2} \log \left(\operatorname{det}\left\{\frac{J(\hat{\boldsymbol{\alpha}})}{2 \pi}\right\}\right) .
\end{aligned}
$$


Assim, a log-verossimilhança perfilada ajustada $h_{A}$ pode ser vista como uma aproximação para a log-verossimilhança restrita de $\xi$.

\section{Desenvolvimento da expressão (2.2.6)}

Temos que

$$
\begin{aligned}
h= & \sum_{k=1}^{l} d_{(k)} \log \lambda_{0}\left(y_{(k)}\right)+\sum_{i=1}^{q} \sum_{j=1}^{n_{i}} \delta_{i j}\left(\mathbf{x}_{\mathbf{i j}}^{\mathbf{T}} \boldsymbol{\beta}+w_{i}\right)- \\
& -\sum_{i=1}^{q} \sum_{j=1}^{n_{i}} \Lambda_{0}\left(y_{i j}\right) \exp \left\{\mathbf{x}_{\mathbf{i j}}^{\mathbf{T}} \boldsymbol{\beta}+w_{i}\right\}+\sum_{i=1}^{q} \log \left(f\left(w_{i}, \xi\right)\right) .
\end{aligned}
$$

Substituindo $\Lambda_{0}(t)$ pelo estimador

$$
\hat{\Lambda}_{0}(t)=\sum_{k: y_{(k)} \leq t} \hat{\lambda}_{0}\left(y_{(k)}\right)=\sum_{k: y_{(k)} \leq t}\left\{\frac{d_{(k)}}{\sum_{R\left(y_{(k)}\right)} \exp \left(\mathbf{x}_{\mathbf{i}}^{\mathbf{T}} \boldsymbol{\beta}+w_{i}\right)}\right\}
$$

obtemos

$$
\begin{aligned}
h^{*}= & \sum_{k=1}^{l} d_{(k)} \log \left\{\frac{d_{(k)}}{\sum_{R\left(y_{(k)}\right)} \exp \left(\mathbf{x}_{\mathbf{i j}}^{\mathbf{T}} \boldsymbol{\beta}+w_{i}\right)}\right\}+\sum_{i=1}^{q} \sum_{j=1}^{n_{i}} \delta_{i j}\left(\mathbf{x}_{\mathbf{i j}}^{\mathbf{T}} \boldsymbol{\beta}+w_{i}\right)- \\
& -\sum_{i=1}^{q} \sum_{j=1}^{n_{i}}\left[\exp \left\{\mathbf{x}_{\mathbf{i j}}^{\mathbf{T}} \boldsymbol{\beta}+w_{i}\right\} \sum_{k: y_{(k)} \leq y_{i j}}\left\{\frac{d_{(k)}}{\sum_{R\left(y_{(k)}\right)} \exp \left(\mathbf{x}_{\mathbf{i j}}^{\mathbf{T}} \boldsymbol{\beta}+w_{i}\right)}\right\}+\right. \\
& +\sum_{i=1}^{q} \log \left(f\left(w_{i}, \xi\right)\right) \\
= & \sum_{k=1}^{l} d_{(k)} \log d_{(k)}-\sum_{k=1}^{l} d_{(k)} \log \left\{\sum_{R\left(y_{(k)}\right)} \exp \left(\mathbf{x}_{\mathbf{i j}}^{\mathbf{T}} \boldsymbol{\beta}+w_{i}\right)\right\}+ \\
& +\sum_{i=1}^{q} \sum_{j=1}^{n_{i}} \delta_{i j}\left(\mathbf{x}_{\mathbf{i j}}^{\mathbf{T}} \boldsymbol{\beta}+w_{i}\right)+\sum_{i=1}^{q} \log \left(f\left(w_{i}, \xi\right)\right)- \\
& -\sum_{k=1}^{l}\left\{\frac{d_{(k)}}{\left.\sum_{R\left(y_{(k)}\right)} \exp _{\left(\mathbf{x}_{\mathbf{i j}}^{\mathbf{T}} \boldsymbol{\beta}+w_{i}\right)} \sum_{R\left(y_{(k)}\right)} \exp \left(\mathbf{x}_{\mathbf{i j}}^{\mathbf{T}} \boldsymbol{\beta}+w_{i}\right)\right\}}\right.
\end{aligned}
$$




$$
\begin{aligned}
= & \sum_{k=1}^{l} d_{(k)} \log d_{(k)}+\sum_{i=1}^{q} \sum_{j=1}^{n_{i}} \delta_{i j}\left(\mathbf{x}_{\mathbf{i j}}^{\mathbf{T}} \boldsymbol{\beta}+w_{i}-\log \left\{\sum_{R\left(y_{(k)}\right)} \exp \left(\mathbf{x}_{\mathbf{i j}}^{\mathbf{T}} \boldsymbol{\beta}+w_{i}\right)\right\}\right) \\
& +\sum_{k=1}^{l} d_{(k)}+\sum_{i=1}^{q} \log \left(f\left(w_{i}, \xi\right)\right) \\
= & \sum_{i=1}^{q} \sum_{j=1}^{n_{i}} \delta_{i j}\left(\mathbf{x}_{\mathbf{i j}}^{\mathbf{T}} \boldsymbol{\beta}+w_{i}-\log \left\{\sum_{R\left(y_{(k)}\right)} \exp \left(\mathbf{x}_{\mathbf{i j}}^{\mathbf{T}} \boldsymbol{\beta}+w_{i}\right)\right\}\right)+\sum_{i=1}^{q} \log \left(f\left(w_{i}, \xi\right)\right)+ \\
& +\sum_{k=1}^{l} d_{(k)}\left(\log d_{(k)}-1\right) .
\end{aligned}
$$

Assim, dado (2.1.1), para o modelo de fragilidade Gama e Log-normal, $h^{*}$ pode ser escrita como

$$
h^{*}=\ell_{P P}+\sum_{k=1}^{l} d_{(k)}\left(\log d_{(k)}-1\right) .
$$

\section{Desenvolvimento da expressão (2.2.7)}

De forma análoga à expressão (2.2.5), temos a seguinte aproximação de Laplace de primeira ordem para a verossimilhança marginal (2.1.3)

$$
\exp \left(\ell_{m}\right) \propto \int \exp \left\{\ell_{P P}\right\} d \mathbf{w} \approx 2 \pi \operatorname{det}\left\{J\left(\ell_{P P}, \hat{\mathbf{w}}\right)\right\}^{-\frac{1}{2}} \exp \left\{\ell_{P P}(\hat{\mathbf{w}})\right\}=\exp \left(p_{\mathbf{w}}\left(\ell_{P P}\right)\right)
$$

Observe $\exp \left\{\ell_{P P}\right\}$ é proporcional à verossimilhança completa (2.1.4). Portanto, temos que

$$
p_{\mathbf{w}}\left(\ell_{P P}\right)=\ell_{P P}(\boldsymbol{\beta}, \hat{\mathbf{w}})-\frac{1}{2} \log \left(\operatorname{det}\left\{\frac{J\left(\ell_{P P}, \hat{\mathbf{w}}\right)}{2 \pi}\right\}\right) .
$$


APÊNDICE A 


\section{Apêndice B}

\section{Expressões do Capítulo 4}

Neste apêndice, desenvolvemos algumas expressões apresentadas no Capítulo 4.

\section{Cálculo de $\mathbf{E}(\mathbf{p})$}

Seja

$$
\lambda_{i j}=\lambda_{0} \exp \{\beta q\}
$$

e $u_{i} \sim \operatorname{Gama}(1 / \xi, 1 / \xi)$. Assim, temos

$$
\bar{p}=E(p)=E\left(\exp \left\{-\lambda_{i j} u_{i} t^{*}\right\}\right)=\left(1+\frac{\lambda_{i j} t^{*}}{\frac{1}{\xi}}\right)^{-\frac{1}{\xi}}=\left(1+\lambda_{i j} t^{*} \xi\right)^{-\frac{1}{\xi}},
$$

pois $E\left(\exp \left\{-\lambda_{i j} u_{i} t^{*}\right\}\right)$ é a função geradora de momentos de $u_{i}$. Portanto, isolando $t^{*}$ nessa expressão, temos

$$
t^{*}=\frac{\bar{p}^{-\xi}-1}{\lambda_{i j} \xi}=\frac{\bar{p}^{-\xi}-1}{\lambda_{0} \exp (\beta q) \xi}
$$

\section{Cálculo de $\rho_{r}$ e $\rho_{d}$}

Dado o modelo em (4.2.1), como $T_{i j k} \sim \operatorname{Exp}\left(\lambda_{i j k}\right)$, podemos escrever

$$
T_{i j k}=\lambda_{0} \exp \left\{\eta_{i j k}+w_{i}+v_{i j}\right\} \epsilon_{i j k},
$$

em que $\eta_{i j k}=-\beta x_{i j k}, \lambda_{0}$ é uma constante e $\epsilon_{i j k} \sim \operatorname{Exp}(1)$. Sendo assim, temos 


$$
\begin{aligned}
\mathrm{E}\left(T_{i j k}\right) & =\mathrm{E}\left(\mathrm{E}\left(T_{i j k} \mid w_{i}, v_{i j}\right)\right)= \\
& =\mathrm{E}\left(\lambda_{0} \exp \left\{\eta_{i j k}+w_{i}+v_{i j}\right\} \mathrm{E}\left(\epsilon_{i j k}\right)\right)= \\
& =\lambda_{0} \exp \left\{\eta_{i j k}\right\} \mathrm{E}\left(\exp \left\{w_{i}+v_{i j}\right\}\right)= \\
& =\lambda_{0} \exp \left\{\eta_{i j k}\right\} \mathrm{E}\left(\exp \left\{w_{i}\right\}\right) \mathrm{E}\left(\exp \left\{v_{i j}\right\}\right)= \\
& =\lambda_{0} \exp \left\{\eta_{i j k}\right\} \exp \left\{\frac{\sigma_{r}^{2}}{2}\right\} \exp \left\{\frac{\sigma_{d}^{2}}{2}\right\},
\end{aligned}
$$

$$
\begin{aligned}
\mathrm{E}\left(T_{i j k} T_{i j^{\prime} k^{\prime}}\right) & =\mathrm{E}\left(\mathrm{E}\left(T_{i j k} T_{i j^{\prime} k^{\prime}} \mid w_{i}, v_{i j}, v_{i j^{\prime}}\right)\right)= \\
& =\mathrm{E}\left(\lambda_{0}^{2} \exp \left\{\eta_{i j k}+\eta_{i j^{\prime} k^{\prime}}\right\} \exp \left\{2 w_{i}+v_{i j}+v_{i j^{\prime}}\right\} \mathrm{E}\left(\epsilon_{i j k}\right) \mathrm{E}\left(\epsilon_{i j^{\prime} k^{\prime}}\right)\right)= \\
& =\lambda_{0}^{2} \exp \left\{\eta_{i j k}+\eta_{i j^{\prime} k^{\prime}}\right\} \mathrm{E}\left(\exp \left\{2 w_{i}+v_{i j}+v_{i j^{\prime}}\right\}\right)= \\
& =\lambda_{0}^{2} \exp \left\{\eta_{i j k}+\eta_{i j^{\prime} k^{\prime}}\right\} \mathrm{E}\left(\exp \left\{2 w_{i}\right\}\right) \mathrm{E}\left(\exp \left\{v_{i j}\right\}\right) \mathrm{E}\left(\exp \left\{v_{i j^{\prime}}\right\}\right)= \\
& =\lambda_{0}^{2} \exp \left\{\eta_{i j k}+\eta_{i j^{\prime} k^{\prime}}\right\} \exp \left\{2 \sigma_{r}^{2}\right\} \exp \left\{\frac{\sigma_{d}^{2}}{2}\right\} \exp \left\{\frac{\sigma_{d}^{2}}{2}\right\}= \\
& =\lambda_{0}^{2} \exp \left\{\eta_{i j k}+\eta_{i j^{\prime} k^{\prime}}\right\} \exp \left\{2 \sigma_{r}^{2}\right\} \exp \left\{\sigma_{d}^{2}\right\},
\end{aligned}
$$

$$
\begin{aligned}
\operatorname{Var}\left(T_{i j k}\right)= & \mathrm{E}\left(\operatorname{Var}\left(T_{i j k} \mid u_{i}, v_{i j}\right)\right)+\operatorname{Var}\left(\mathrm{E}\left(T_{i j k} \mid u_{i}, v_{i j}\right)\right)= \\
= & \mathrm{E}\left(\left[\lambda_{0} \exp \left\{\eta_{i j k}\right\} \exp \left\{u_{i}+w_{i j}\right\}\right]^{2} \operatorname{Var}\left(\epsilon_{i j k}\right)\right)+ \\
& +\operatorname{Var}\left(\lambda_{0} \exp \left\{\eta_{i j k}\right\} \exp \left\{u_{i}+w_{i j}\right\} \mathrm{E}\left(\epsilon_{i j k}\right)\right)= \\
= & \lambda_{0}^{2} \exp \left\{2 \eta_{i j k}\right\} \mathrm{E}\left(\exp \left\{2 u_{i}\right\}\right) \mathrm{E}\left(\exp \left\{2 w_{i j}\right\}\right)+ \\
& +\lambda_{0}^{2} \exp \left\{2 \eta_{i j k}\right\} \operatorname{Var}\left(\exp \left\{u_{i}+w_{i j}\right\}\right)= \\
= & \lambda_{0}^{2} \exp \left\{2 \eta_{i j k}\right\} \exp \left\{2 \sigma_{r}^{2}\right\} \exp \left\{2 \sigma_{d}^{2}\right\}+ \\
& +\lambda_{0}^{2} \exp \left\{2 \eta_{i j k}\right\}\left[\mathrm{E}\left(\exp \left\{u_{i}+w_{i j}\right\}^{2}\right)-\mathrm{E}^{2}\left(\exp \left\{u_{i}+w_{i j}\right\}\right)\right]= \\
= & \lambda_{0}^{2} \exp \left\{2 \eta_{i j k}\right\} \exp \left\{2 \sigma_{r}^{2}\right\} \exp \left\{2 \sigma_{d}^{2}\right\}+ \\
& +\lambda_{0}^{2} \exp \left\{2 \eta_{i j k}\right\}\left[\exp \left\{2 \sigma_{r}^{2}\right\} \exp \left\{2 \sigma_{d}^{2}\right\}-\exp \left\{\sigma_{r}^{2}\right\} \exp \left\{\sigma_{d}^{2}\right\}\right]= \\
= & \lambda_{0}^{2} \exp \left\{2 \eta_{i j k}\right\}\left(2 \exp \left\{2 \sigma_{r}^{2}\right\} \exp \left\{2 \sigma_{d}^{2}\right\}-\exp \left\{\sigma_{r}^{2}\right\} \exp \left\{\sigma_{d}^{2}\right\}\right)
\end{aligned}
$$




$$
\begin{aligned}
\operatorname{Cov}\left(T_{i j k}, T_{i j^{\prime} k^{\prime}}\right) & =\mathrm{E}\left(T_{i j k} T_{i j^{\prime} k^{\prime}}\right)-\mathrm{E}\left(T_{i j k}\right) \mathrm{E}\left(T_{i j^{\prime} k^{\prime}}\right)= \\
& =\lambda_{0}^{2} \exp \left\{\eta_{i j k}+\eta_{i j^{\prime} k^{\prime}}\right\}\left(\exp \left\{2 \sigma_{r}^{2}\right\} \exp \left\{\sigma_{d}^{2}\right\}-\exp \left\{\sigma_{r}^{2}\right\} \exp \left\{\sigma_{d}^{2}\right\}\right)
\end{aligned}
$$

Dessa forma, temos, $\quad j \neq j^{\prime}$,

$$
\begin{aligned}
\rho_{r} & =\operatorname{Corr}\left(T_{i j k}, T_{i j^{\prime} k^{\prime}}\right)=\frac{\operatorname{Cov}\left(T_{i j k}, T_{i j^{\prime} k^{\prime}}\right)}{\sqrt{\operatorname{VAR}\left(T_{i j k}\right) \operatorname{VAR}\left(T_{i j^{\prime} k^{\prime}}\right)}}= \\
& =\frac{\lambda_{0}^{2} \exp \left\{\eta_{i j k}+\eta_{i j^{\prime} k^{\prime}}\right\}\left(\exp \left\{2 \sigma_{r}^{2}\right\} \exp \left\{\sigma_{d}^{2}\right\}-\exp \left\{\sigma_{r}^{2}\right\} \exp \left\{\sigma_{d}^{2}\right\}\right)}{\sqrt{\lambda_{0}^{4} \exp \left\{2 \eta_{i j k}+2 \eta_{i j^{\prime} k^{\prime}}\right\}\left(2 \exp \left\{2 \sigma_{r}^{2}\right\} \exp \left\{2 \sigma_{d}^{2}\right\}-\exp \left\{\sigma_{r}^{2}\right\} \exp \left\{\sigma_{d}^{2}\right\}\right)^{2}}}= \\
& =\frac{\exp \left\{\sigma_{r}^{2}\right\}-1}{2 \exp \left\{\sigma_{r}^{2}\right\} \exp \left\{\sigma_{d}^{2}\right\}-1} .
\end{aligned}
$$

De forma análoga, temos

$$
\rho_{d}=\operatorname{Corr}\left(T_{i j k}, T_{i j k^{\prime}}\right)=\frac{\exp \left\{\sigma_{r}^{2}\right\} \exp \left\{\sigma_{d}^{2}\right\}-1}{2 \exp \left\{\sigma_{r}^{2}\right\} \exp \left\{\sigma_{d}^{2}\right\}-1}
$$


APÊNDICE B 


\section{Apêndice C}

\section{Gráficos}

Neste apêndice, apresentaremos os gráficos das estimativas dos parâmetros encontradas nas simulações Gama (com um componente de fragilidade) e Log-normal (com dois componentes de fragilidade).

\section{C.1 Gráficos da simulação Gama}

Nas Figuras C.1 a C.8 estão apresentados os gráficos para as estimativas de $\beta$, ao longo das 1000 réplicas, referentes a todos os cenários considerados. Nas Figuras C.9 a C.16 estão apresentados os gráficos para as estimativas de $\xi$, ao longo das 1000 réplicas, referentes a todos os cenários considerados. Cada gráfico corresponde a uma combinação de $p$ e $\tau$ e é composto por 9 janelas, sendo cada uma delas uma combinação de valores de $n i$ e $k$. Janelas vazias correspondem a cenários não considerados e a reta representa o verdadeiro valor do parâmetro. 


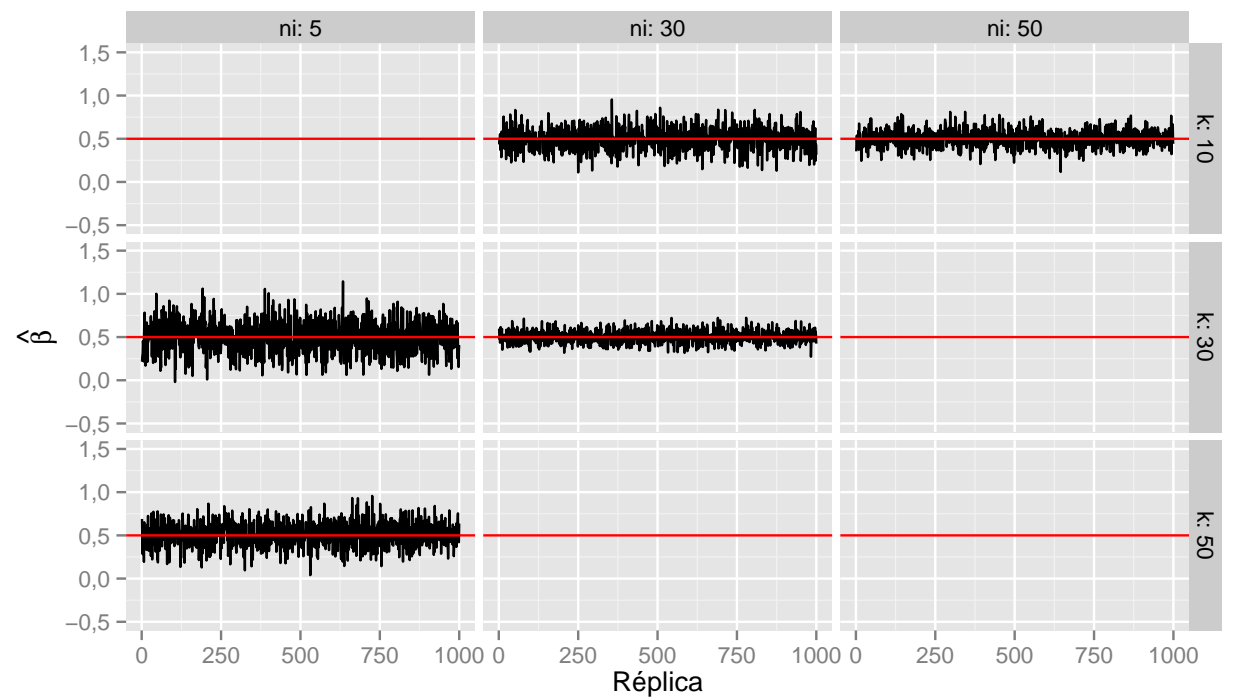

Figura C.1: Estimativas de $\beta$ da abordagem penalizada. Cenários com $p=0,15$ e $\tau=0,01$.

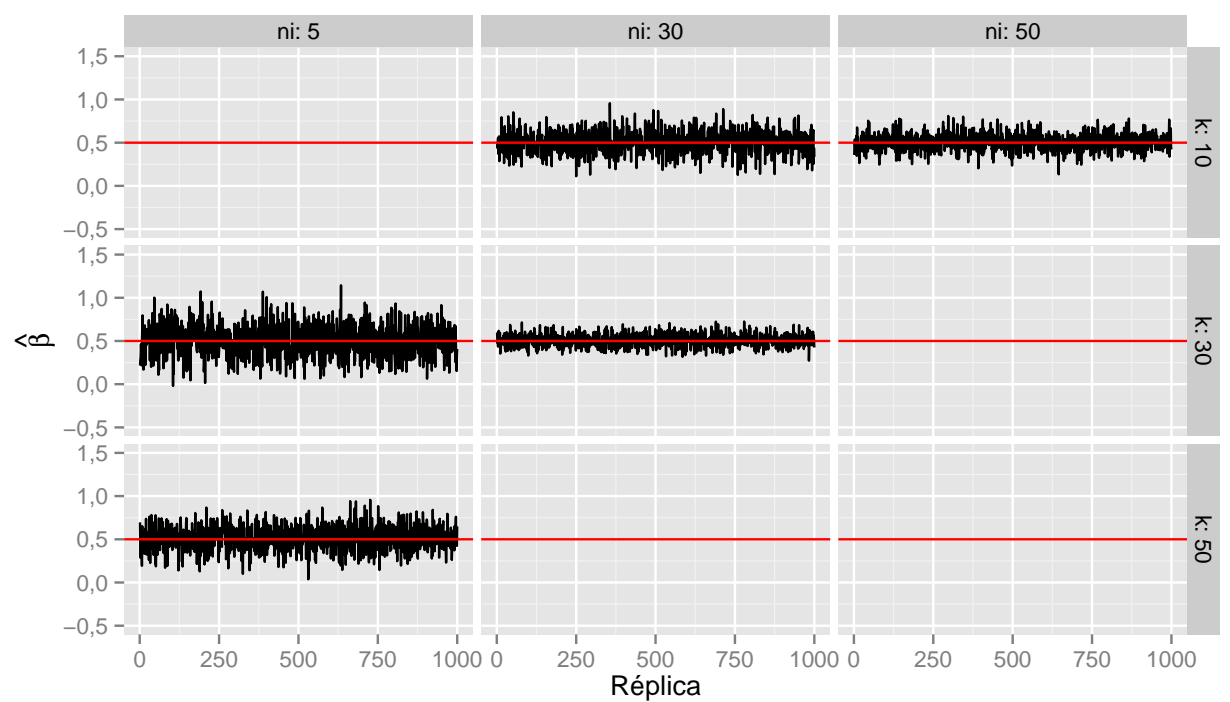

Figura C.2: Estimativas de $\beta$ da abordagem hierárquica. Cenários com $p=0,15$ e $\tau=0,01$. 


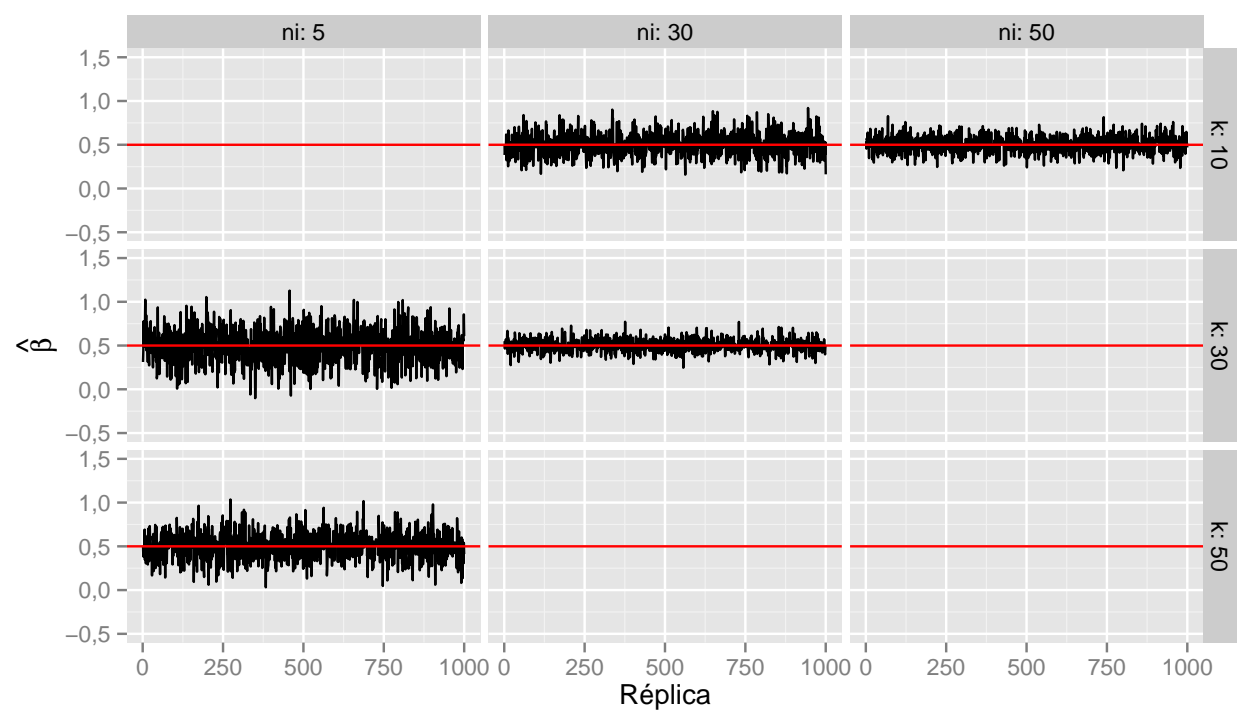

Figura C.3: Estimativas de $\beta$ da abordagem penalizada. Cenários $\operatorname{com} p=0,15$ e $\tau=0,1$.

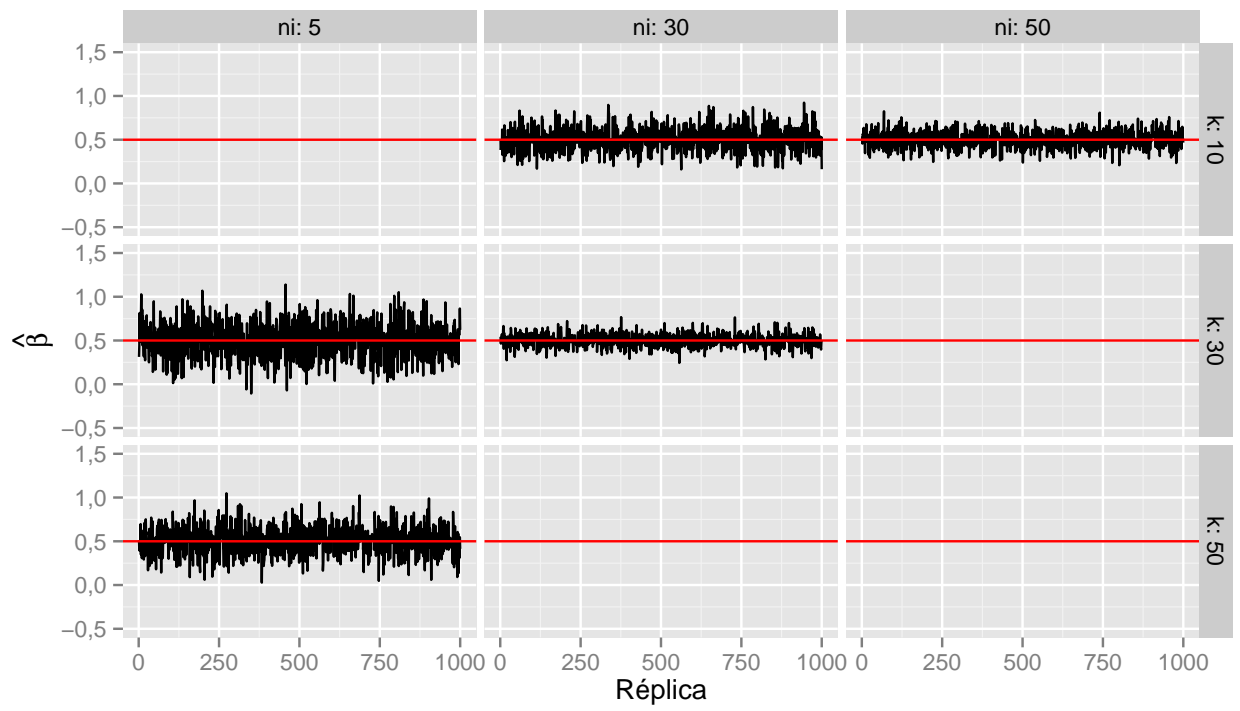

Figura C.4: Estimativas de $\beta$ da abordagem hierárquica. Cenários com $p=0,15$ e $\tau=0,1$. 


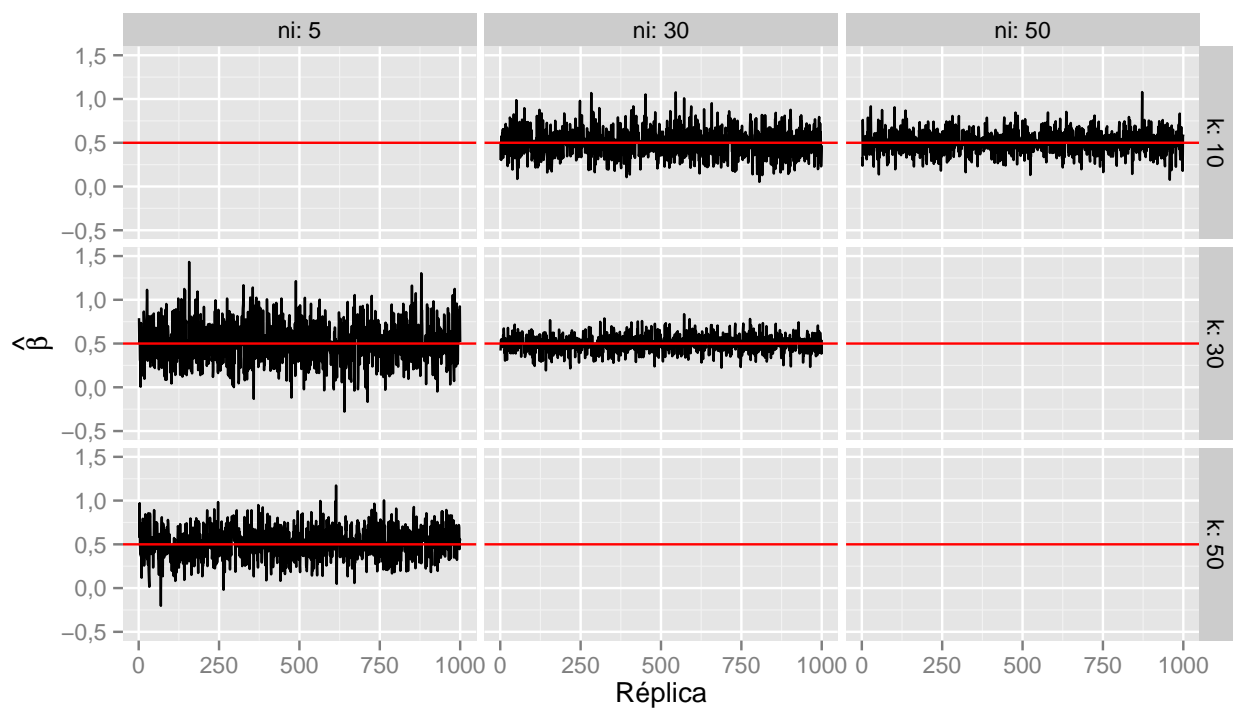

Figura C.5: Estimativas de $\beta$ da abordagem penalizada. Cenários $\operatorname{com} p=0,5$ e $\tau=0,01$.

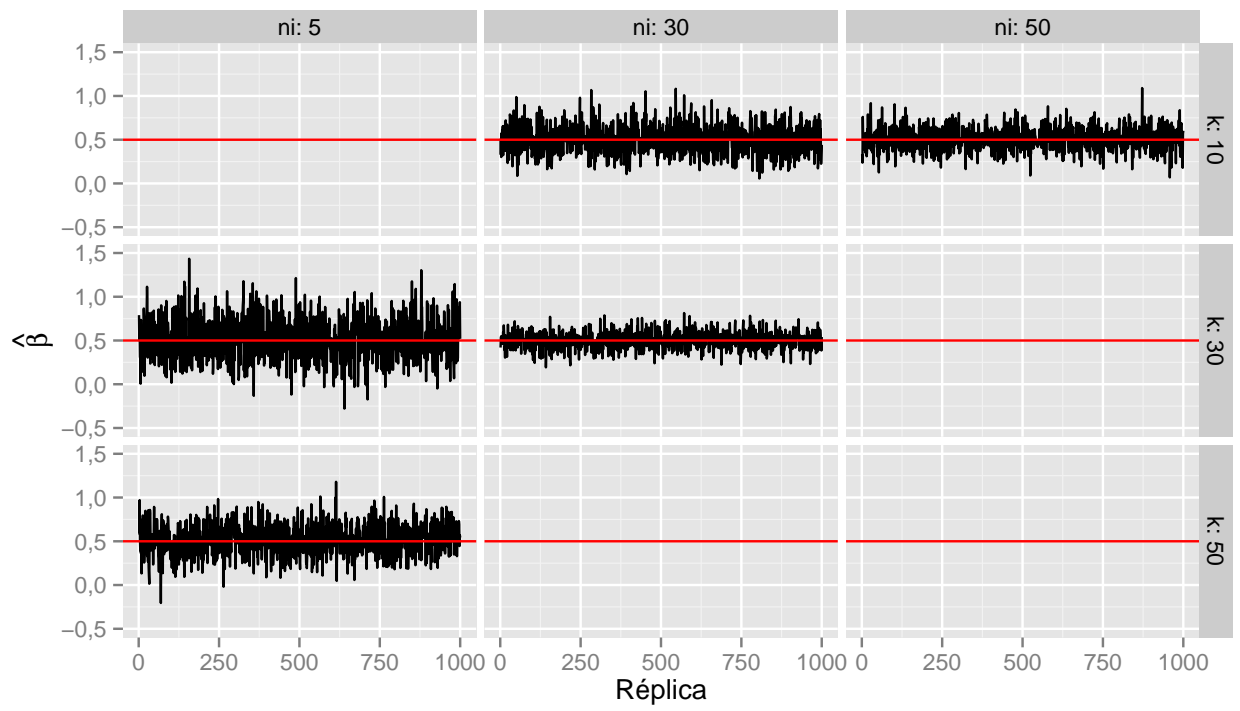

Figura C.6: Estimativas de $\beta$ da abordagem hierárquica. Cenários com $p=0,5$ e $\tau=$ 0,01 . 


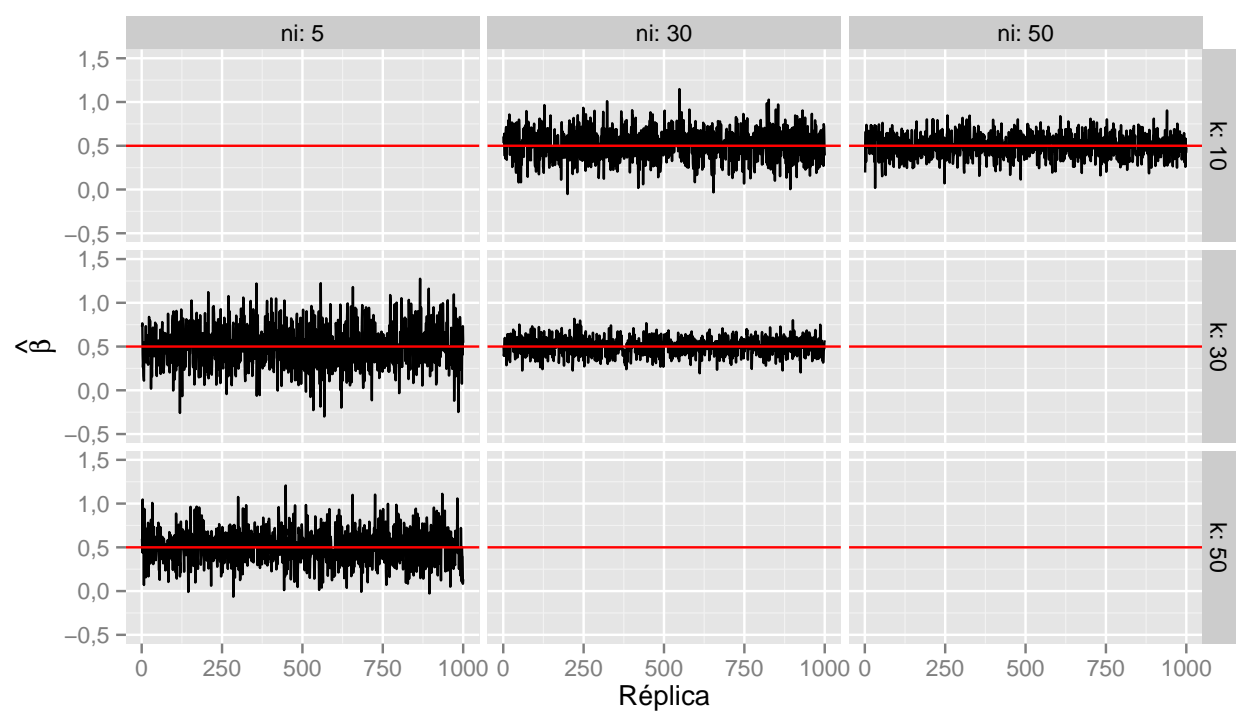

Figura C.7: Estimativas de $\beta$ da abordagem penalizada. Cenários com $p=0,5$ e $\tau=0,1$.

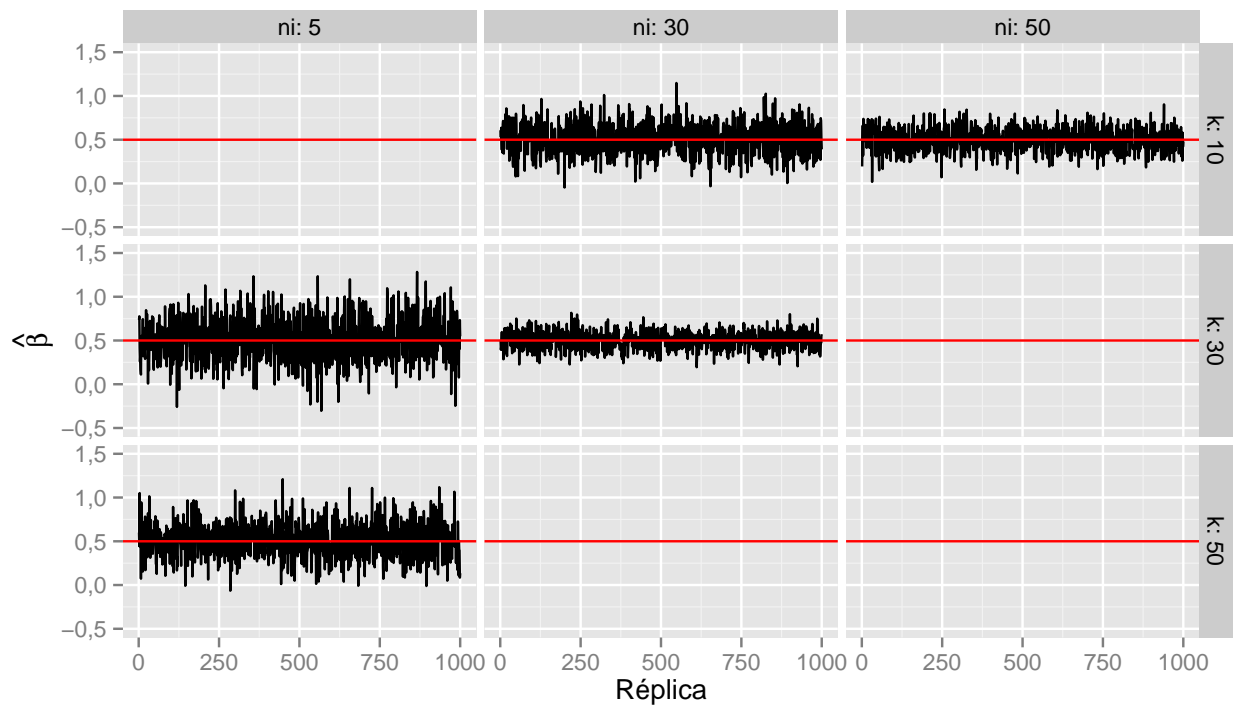

Figura C.8: Estimativas de $\beta$ da abordagem hierárquica. Cenários com $p=0,5$ e $\tau=0,1$. 


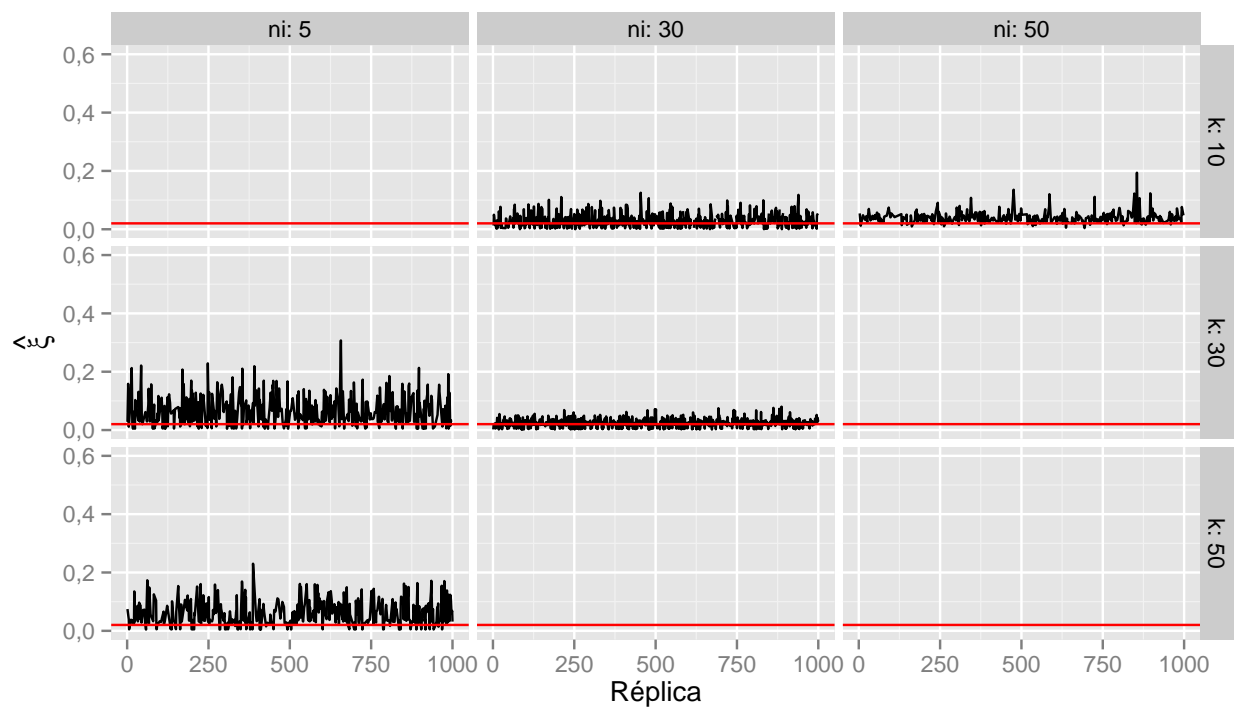

Figura C.9: Estimativas de $\xi$ da abordagem penalizada. Cenários com $p=0,15$ e $\tau=$ 0,01 .

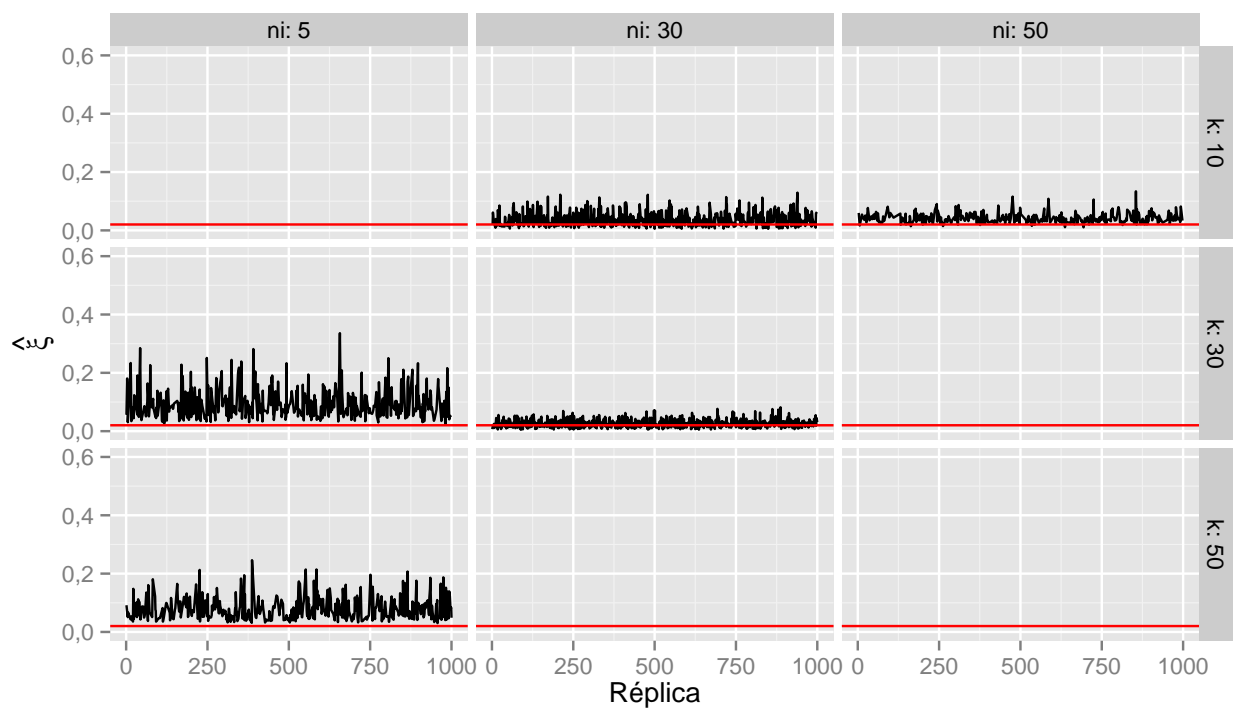

Figura C.10: Estimativas de $\xi$ da abordagem hierárquica. Cenários com $p=0,15$ e $\tau=0,01$. 


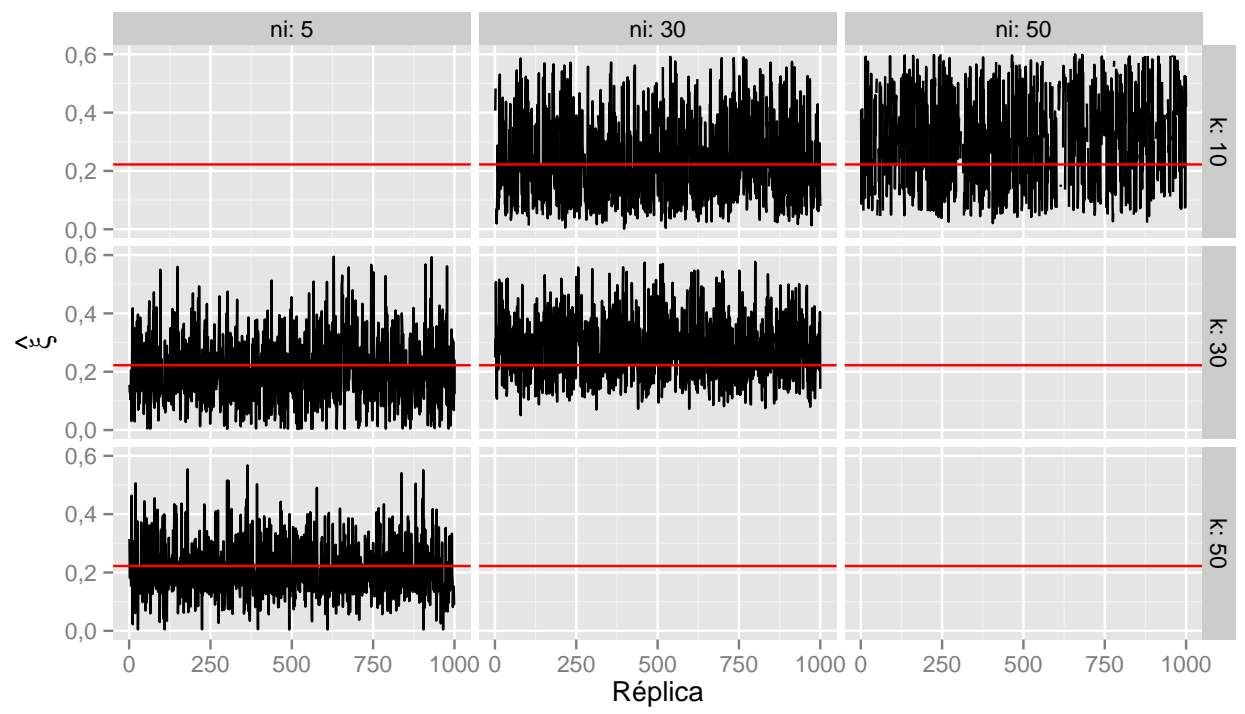

Figura C.11: Estimativas de $\xi$ da abordagem penalizada. Cenários com $p=0,15$ e $\tau=0,1$.

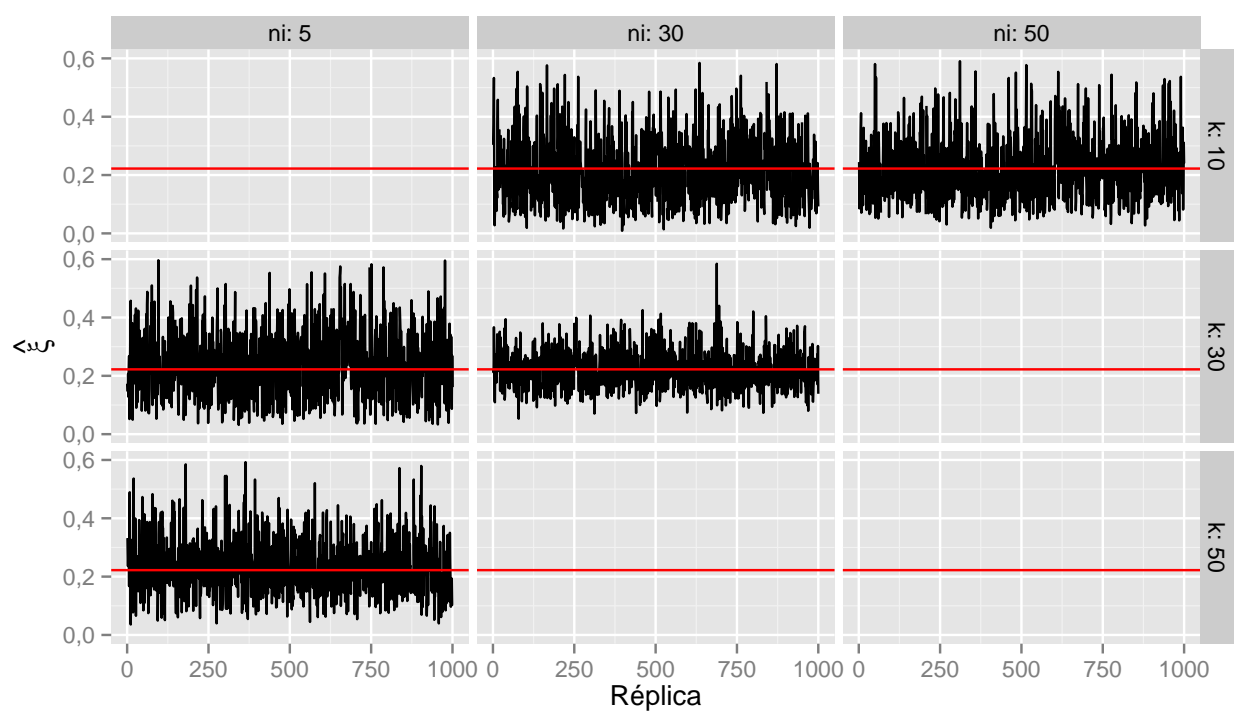

Figura C.12: Estimativas de $\xi$ da abordagem hierárquica. Cenários com $p=0,15$ e $\tau=0,1$. 


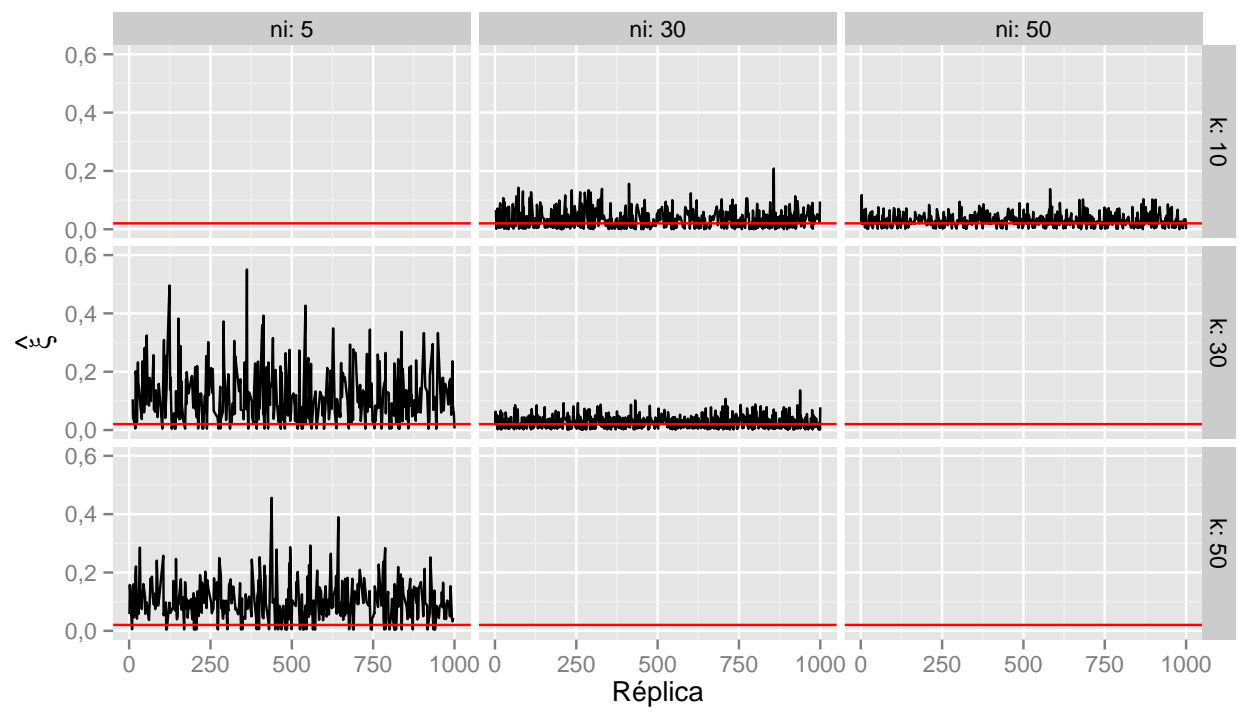

Figura C.13: Estimativas de $\xi$ da abordagem penalizada. Cenários com $p=0,5$ e $\tau=$ 0,01 .

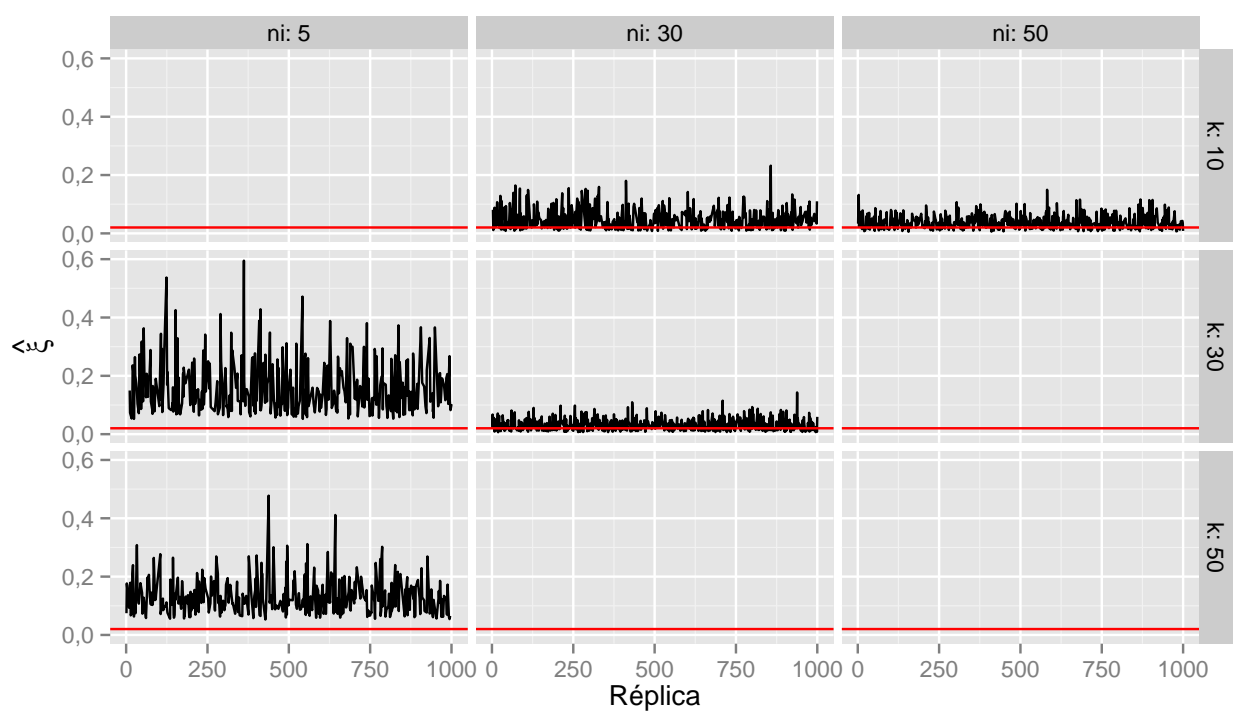

Figura C.14: Estimativas de $\xi$ da abordagem hierárquica. Cenários com $p=0,5$ e $\tau=0,01$. 


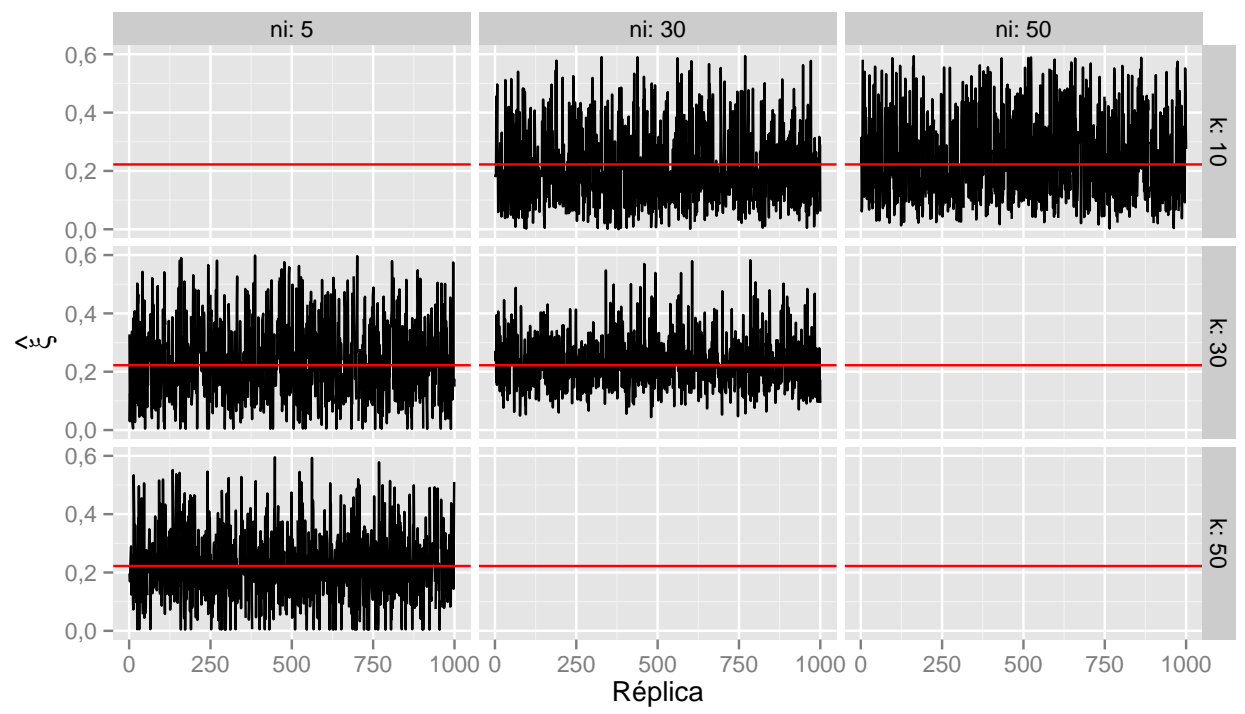

Figura C.15: Estimativas de $\xi$ da abordagem penalizada. Cenários com $p=0,5$ e $\tau=0,1$.

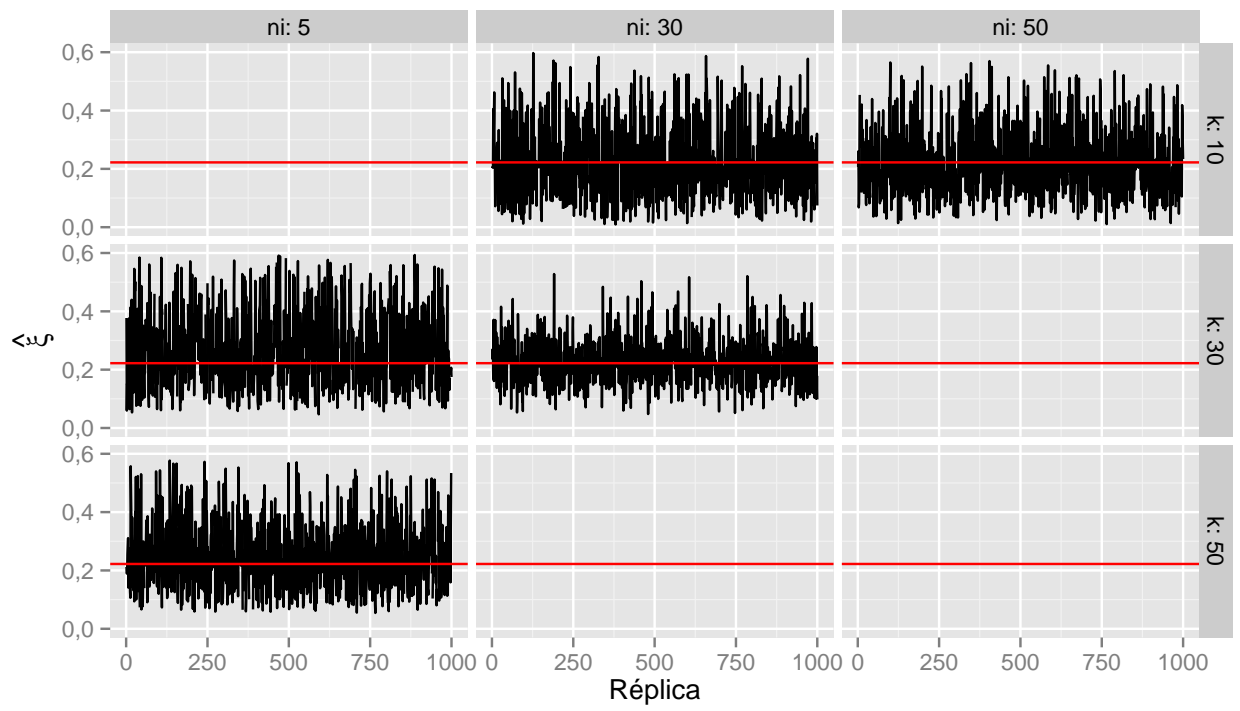

Figura C.16: Estimativas de $\xi$ da abordagem hierárquica. Cenários com $p=0,5$ e $\tau=0,1$. 


\section{C.2 Gráficos da simulação Log-normal}

Nas Figuras C.17 e C.18 estão apresentados os gráficos para as estimativas de $\beta$, ao longo das 1000 réplicas, referentes aos cenários considerados. Nas Figuras C.19 a C.22 estão apresentados os gráficos para as estimativas de $\sigma_{r}^{2}$ e $\sigma_{d}^{2}$, ao longo das 1000 réplicas, referentes a todos os cenários considerados. 


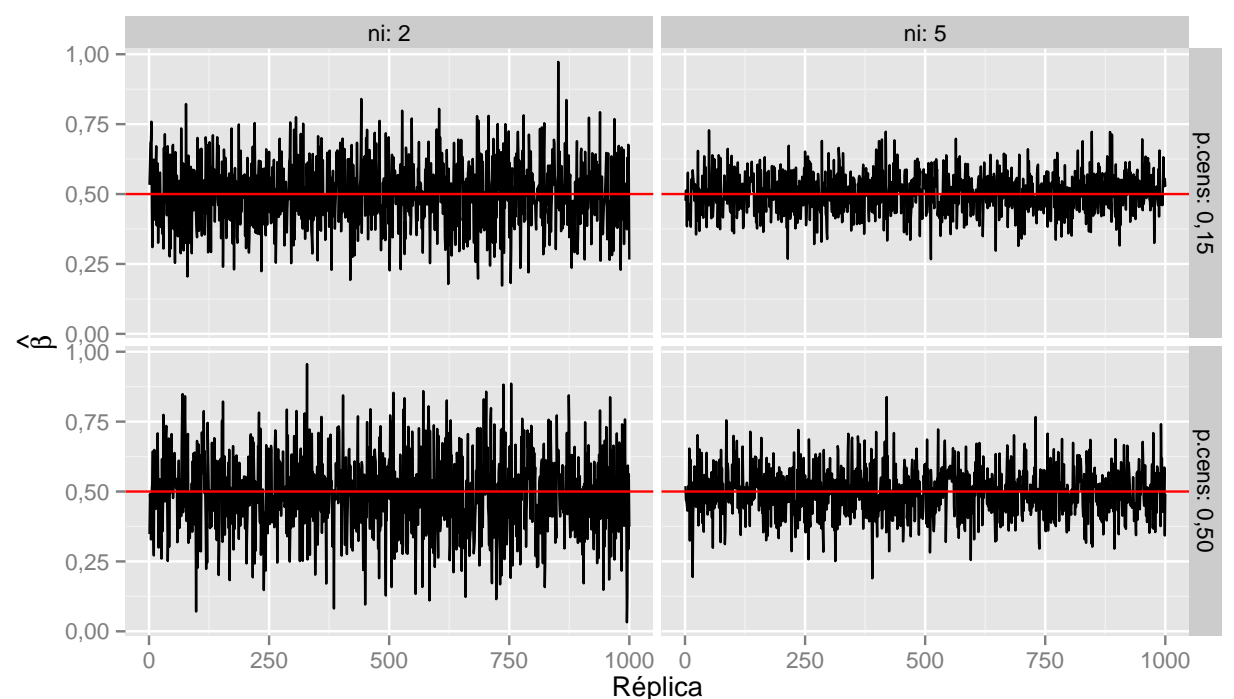

Figura C.17: Estimativas de $\beta$ da abordagem penalizada.

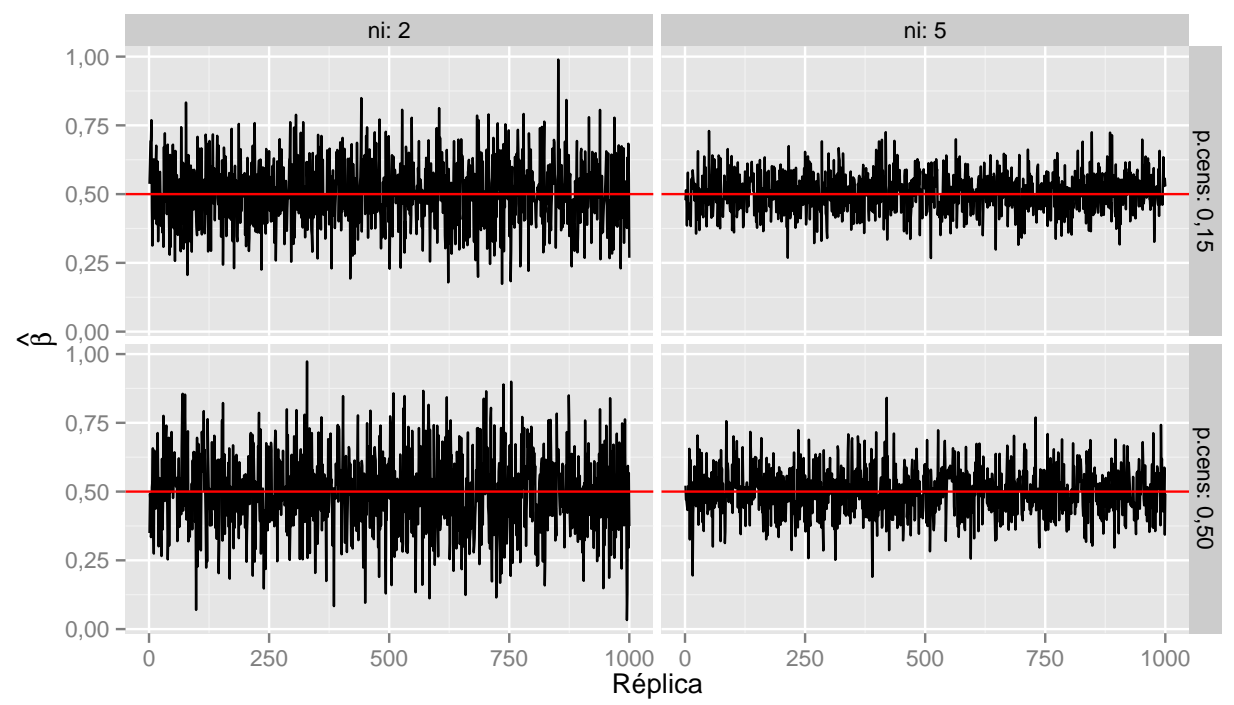

Figura C.18: Estimativas de $\beta$ da abordagem hierárquica. 


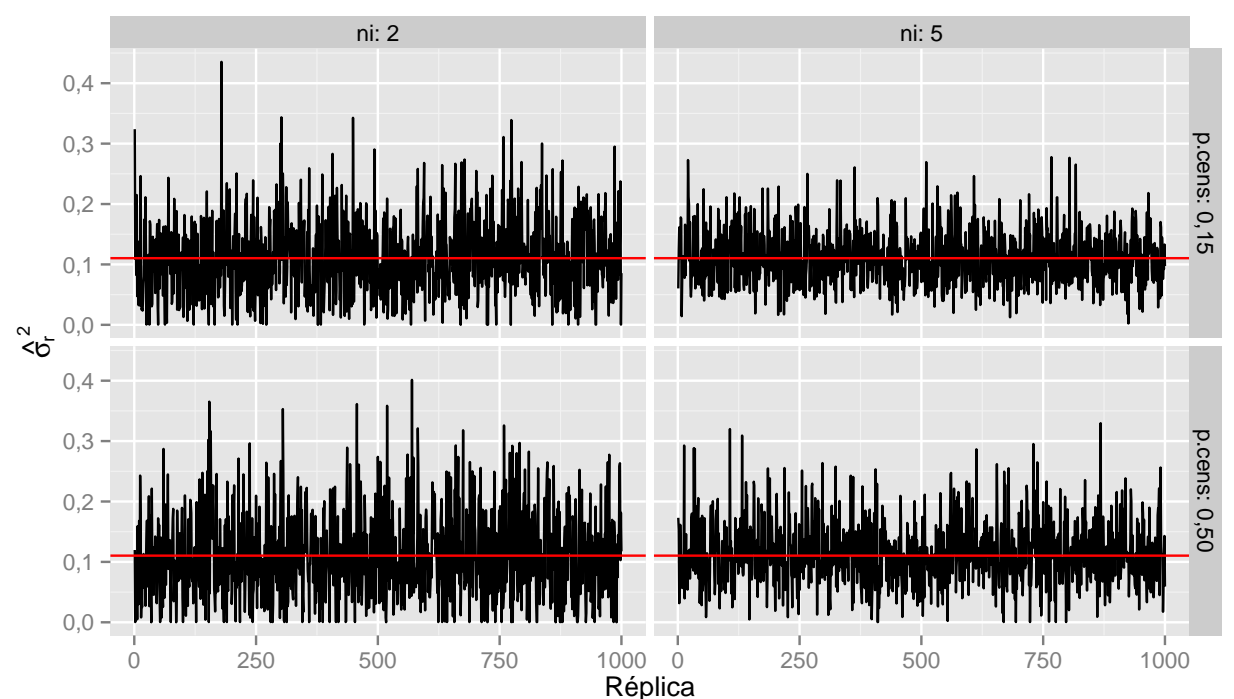

Figura C.19: Estimativas de $\sigma_{r}^{2}$ da abordagem penalizada.

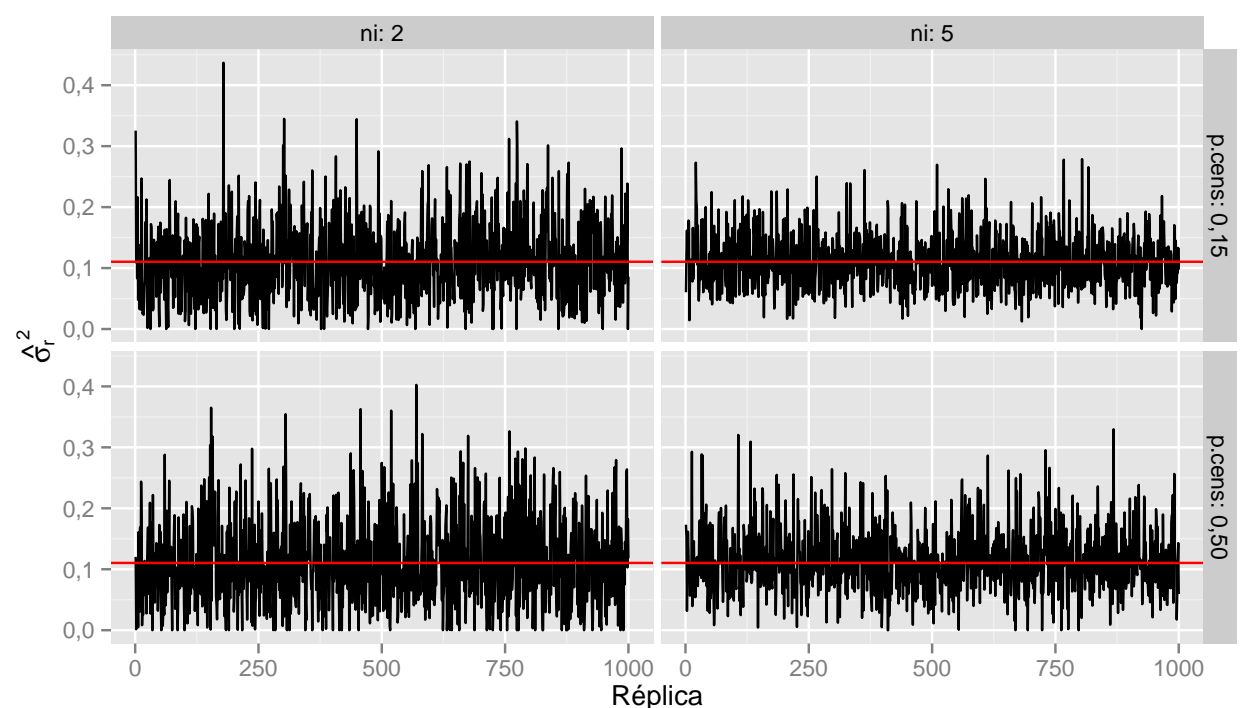

Figura C.20: Estimativas de $\sigma_{r}^{2}$ da abordagem hierárquica. 


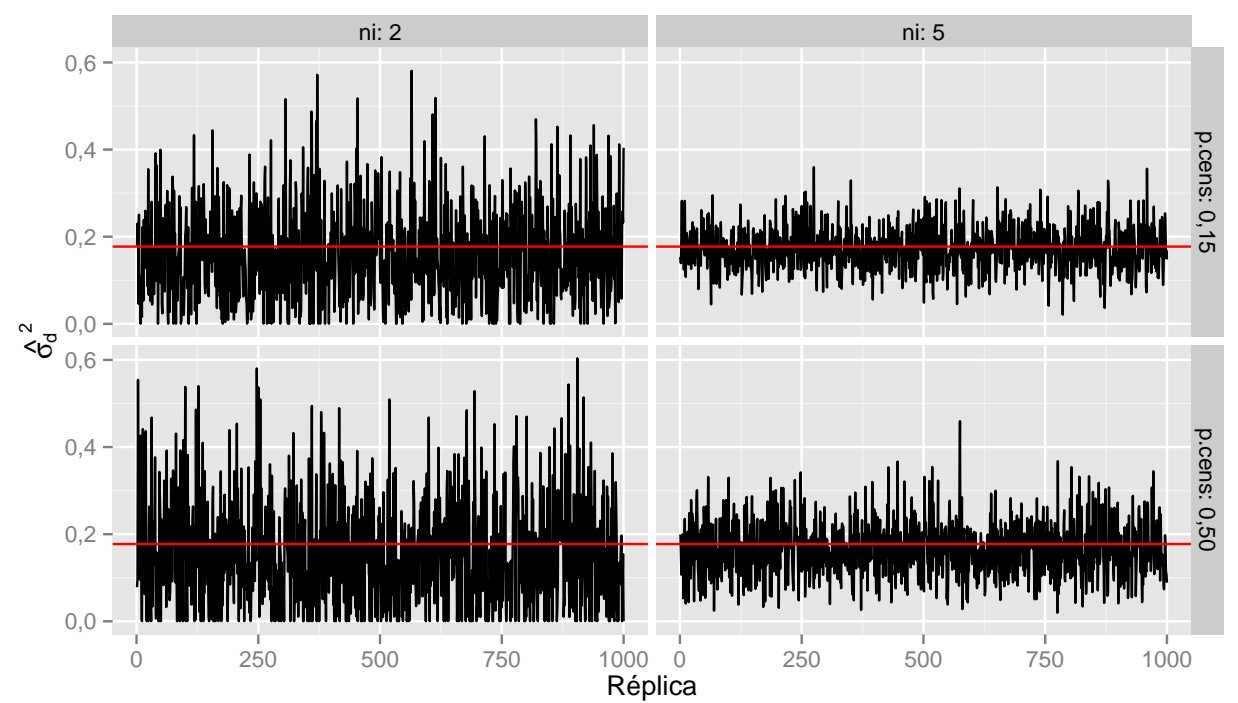

Figura C.21: Estimativas de $\sigma_{d}^{2}$ da abordagem penalizada.

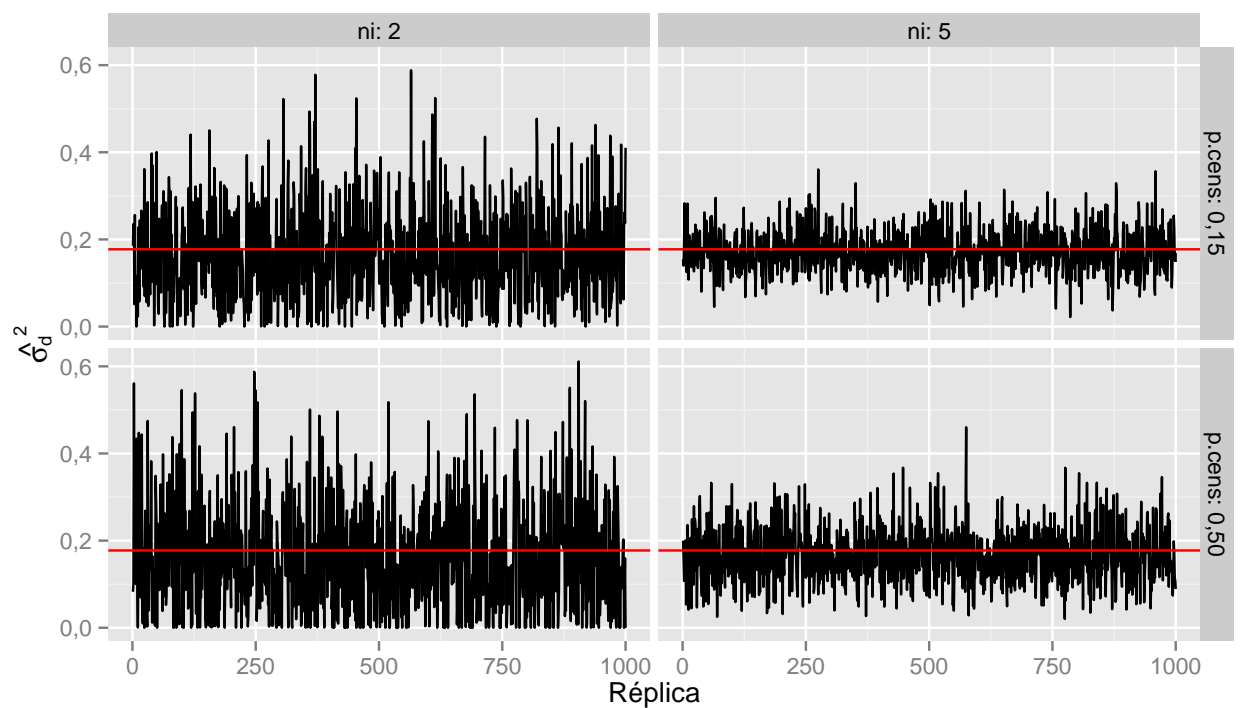

Figura C.22: Estimativas de $\sigma_{d}^{2}$ da abordagem hierárquica. 
APÊNDICE C 


\section{Referências Bibliográficas}

Aslanidou et al.(1998) H. Aslanidou, D. K. Dey e D. Sinha. Bayesian analysis of multivariate survival data using monte carlo methods. Can J. Statist., 26:33-48.

Barndorff-Nielsen e $\operatorname{Cox}(\mathbf{1 9 8 9})$ O. E. Barndorff-Nielsen e D. R. Cox. Asymptotic Techniques for Use in Statistics. Monographs on statistics and applied probability. "Chapman and Hall, London.

Breslow(1974) N. E. Breslow. Covariance analysis of censored survival data. Biometrics, 30(1):89-99.

Breslow e Clayton(1993) N. E. Breslow e D. G. Clayton. Approximate inference in generalized linear mixed models. Journal of the American Statistical Association, 88: $9-25$.

Clayton(1991) D. G. Clayton. A monte carlo method for bayesian inference in frailty models. Biometrica, 47:467-485.

Clayton(1978) David G. Clayton. A model for association in bivariate life tables and its application in epidemiological studies of familial tendency disease incidence. Biometrika, 65:141-151.

Colosimo e Giolo(2006) Enrico Antônio Colosimo e Suely Ruiz Giolo. Análise de Sobrevivência Aplicada. Edgard Blucher, São Paulo.

$\operatorname{Cox}(1972)$ D. R. Cox. Regression models and life-tables (with discussion). J. R. Statist. Soc., B, 34:187-220.

Cox e Reid(1987) D. R. Cox e N. Reid. Parameter orthogonality and approximate conditional inference. Journal of the Royal Statistical Society, 49(1):1-39.

Cui e Sun(2004) Sufang Cui e Yanqing Sun. Checking for de gamma frailty distribution under the marginal proportional hazards frailty model. Statistica Sinica, 14:249-267.

Geerdens et al.(2013) Candida Geerdens, Gerda Claeskens e Paul Janssen. Goodnessof-fit tests for the frailty distribution in proportional hazards models with shared frailty. Biostatistics, 14(3):433-446.

Glidden(1999) David V. Glidden. Checking the adequacy of the gamma frailty model for multivariate failure times. Biometrika, 86(2):381-393. 
Good e Gaskins(1971) I. J. Good e R. A. Gaskins. Nonparametric roughness penalties for probability densities. Biometrika, 58:255-277.

Ha e Lee(2005) Il Do Ha e Youngjo Lee. Comparison of hierarchical likelihood versus orthodox best linear unbiased predictor approaches for frailty models. Biometrika, 92 (3):717-723.

Ha et al.(2001) Il Do Ha, Youngjo Lee e Jae-Kee Song. Hierarchical likelihood approach for frailty models. Biometrika, 88:233-243.

Ha et al.(2010) Il Do Ha, Maegseok Noh e Youngjo Lee. Bias reduction of likelihood estimators in semiparametric frailty models. Scandinavian Journal of Statistics, 37: 307-320.

Ha et al.(2012) Il Do Ha, Maegseok Noh e Youngjo Lee. Frailtyhl: a package for fitting frailty models with h-likelihood. The $R$ Journal, 4(2):28-36.

Harville(1977) D. Harville. Maximum likelihood approaches to variance component estimation and related problems. Journal of the American Statistical Association, 72: $320-340$.

Hougaard(1987) P. Hougaard. Modeling multivariate suvival. Scond. J. Statist., 14: $291-304$.

Hougaard(2000) P. Hougaard. Analysis of multivariate survival data. Statistics for Biology and Health. Springer, New York.

Hougaard(1986) Philip Hougaard. Survival models for heterogeneous populations derived from stable distributions. Biostatistics, 73(2):387-396.

Johansen(1983) Soren Johansen. An extension of cox's regression model. International Statistical Review, 51(2):165-174.

Kalbfleisch e Prentice(2002) J. D. Kalbfleisch e R. L. Prentice. The Statistical Analysis of Failure Time Data. Wiley Series in Probability and Statistics. J. Wiley.

Klein(1992) J. P. Klein. Semiparametric estimation of random effects using the cox model based on the em algorithm. Biometrica, 49:221-225.

Lawless(2002) J. F. Lawless. Statistical Models and Methods for Lifetime Data. WileyInterscience, New York.

Lee e Nelder(1996) Y. Lee e J. A. Nelder. Hierarchical generalized linear models (with discussion). J. R. Statist. Soc., B 58(4):619-678.

Lee et al.(2006) Youngjo Lee, John A. Nelder e Yudi Pawitan. Generalized Linear Models with Random Effects: unified analysis via H-likelihood. Chapman and Hall/CRC, Boca Raton. 
McGilchrist(1993) C. A. McGilchrist. Reml estimation for survival models with frailty. Biometrica, 49:221-225.

McGilchrist e Aisbett(1991) C. A. McGilchrist e C. W. Aisbett. Regression with frailty in survival analysis. Biometrica, 47:461-466.

Nielsen et al.(1992) G. G. Nielsen, R. D. Gill, P. K. Andersen e T. I. A. Sorensen. A counting process approch to maximum likehood estimation in frailty models. Scand. J. Statist., 19:25-44.

Oakes(1982) David Oakes. A model for association in bivariate survival data. Journal of the Royal Statistical Society. Series B (Methodological), 44(3):414-422.

Oakes(1989) David Oakes. Bivariate survival models induced by frailties. Journal of the American Statistical Association, 84(406):487-493.

Parner(1998) E. Parner. Asymptotic theory for the correlated gamma-frailty model. Ann. Statist., 26:181-214.

Patterson e Thompson(1971) H.D. Patterson e R. Thompson. Recovery of interblock information when block sizes are unequal. Biometrika, 58:545-554.

Pinheiro e Chao(2006) José C. Pinheiro e Edward C. Chao. Efficient laplacian multilevel quadrature algorithms for generalized linear mixed models. Journal of Computational and Graphical Statistics, 15(1):58-81.

Ripatti e Palmgren(2000) Samuli Ripatti e Juni Palmgren. Estimation of multivariate frailty models using penalized partial likelihood. Biometrics, 56:1016-1022.

Rondeau et al.(2003) Virgine Rondeau, Daniel Commenges e Pierre Joly. Maximum penalized likelihood estimation in a gamma-frailty model. Lifetime Data Analysis, 9: 139-153.

Therneau e Grambsch(2000) T. M. Therneau e Patricia M. Grambsch. Modeling survival data: extending the Cox model. Springer: Statistics for Biology and Health, New York.

Therneau et al.(2003) T. M. Therneau, Patricia M. Grambsch e V. S. Pankratz. Penalized survival models and frailty. Journal of Computational and Graphical Statistics, $12: 1: 156-175$.

Tunes e de Lima(2005) Gisela Tunes e Antonio Carlos Pedroso de Lima. A bootstrap evaluation of wald test for the variance of random effects in survival analysis. Relatório Técnico RT-MAE 2005-28, Instituto de Matemática e Estatística da Universidade de São Paulo.

Vaupel et al.(1979) James W. Vaupel, Kenneth G. Manton e Eric Stallard. The impact of heterogeneity in individual frailty on the dynamics of mortality. DEMOGRAPHY, 16(3):439-454. 